\title{
Guidelines for DNA recombination and repair studies: Cellular assays of DNA repair pathways
}

\author{
Hannah L. Klein ${ }^{1, *}$, Giedrè Bačinskaja ${ }^{2}$, Jun $\mathrm{Che}^{3}$, Anais Cheblal ${ }^{4}$, Rajula Elango ${ }^{5}$, Anastasiya Epshtein ${ }^{1}$, \\ Devon M. Fitzgerald ${ }^{6-9}$, Belén Gómez-González ${ }^{10}$, Sharik R. Khan ${ }^{11}$, Sandeep Kumar ${ }^{7}$, Bryan A. Leland ${ }^{12}$, Léa \\ Marie $^{13}$, Qian $\mathrm{Mei}^{14}$, Judith Miné-Hattab ${ }^{16,17}$, Alicja Piotrowska ${ }^{18}$, Erica J. Polleys ${ }^{19}$, Christopher D. \\ Putnam $^{20,21}$, Elina A. Radchenko ${ }^{19}$, Anissia Ait Saada ${ }^{22,23}$, Cynthia J. Sakofsky ${ }^{24}$, Eun Yong Shim ${ }^{3}$, Mathew \\ Stracy ${ }^{25}$, Jun Xia ${ }^{6-9}$, Zhenxin Yan ${ }^{7}$, Yi Yin $^{26}$, Andrés Aguilera ${ }^{10}$, Juan Lucas Argueso ${ }^{27}$, Catherine $\mathrm{H}$. \\ Freudenreich ${ }^{19,28}$, Susan M. Gasser ${ }^{4}$, Dmitry A. Gordenin ${ }^{24}$, James E. Haber ${ }^{29}$, Grzegorz Ira ${ }^{7}$, Sue \\ Jinks-Robertson ${ }^{30}$, Megan C. King ${ }^{12}$, Richard D. Kolodner ${ }^{20,31-33}$, Andrei Kuzminov ${ }^{11}$, Sarah AE Lambert ${ }^{22,23}$, \\ Sang Eun Lee ${ }^{3}$, Kyle M. Miller ${ }^{6,15}$, Sergei M. Mirkin ${ }^{19}$, Thomas D. Petes ${ }^{26}$, Susan M. Rosenberg ${ }^{6-9,14}$, Rodney \\ Rothstein ${ }^{34}$, Lorraine S. Symington ${ }^{13}$, Pawel Zawadzki ${ }^{18}$, Nayun Kim ${ }^{35, *}$, Michael Lisby ${ }^{2, *}$ and Anna \\ Malkova ${ }^{5, *}$
}

\begin{abstract}
1 Department of Biochemistry and Molecular Pharmacology, New York University School of Medicine, New York, NY, USA. ${ }^{2}$ Department of Biology, University of Copenhagen, DK-2200 Copenhagen N, Denmark. ${ }^{3}$ Department of Radiation Oncology, University of Texas Health Science Center at San Antonio, 7703 Floyd Curl Drive, San Antonio, TX, USA. ${ }^{4}$ Friedrich Miescher Institute for Biomedical Research (FMI), 4058 Basel, Switzerland. ${ }^{5}$ Department of Biology, University of lowa, lowa City, IA, USA. ${ }^{6}$ Dan L Duncan Comprehensive Cancer Center, Baylor College of Medicine, Houston, TX, USA. ${ }^{7}$ Department of Molecular and Human Genetics, Baylor College of Medicine, Houston, TX, USA. ${ }^{8}$ Department of Biochemistry and Molecular Biology, Baylor College of Medicine, Houston, TX, USA. ${ }^{9}$ Department of Molecular Virology and Microbiology, Baylor College of Medicine, Houston, TX, USA. ${ }^{10}$ Centro Andaluz de BIología Molecular y Medicina Regenerativa-CABIMER, Universidad de Sevilla, Seville, Spain. ${ }^{11}$ Department of Microbiology, University of Illinois at Urbana-Champaign, Urbana, IL, USA. ${ }^{12}$ Yale School of Medicine, New Haven, CT, USA. ${ }^{13}$ Department of Microbiology and Immunology, Columbia University Medical Center, New York, NY, USA. ${ }^{14}$ Systems, Synthetic and Physical Biology Graduate Program, Rice University, Houston, TX, USA. ${ }^{15}$ Department of Molecular Biosciences, University of Texas at Austin, Austin, TX, USA. 16 Institut Curie, PSL Research University, CNRS, UMR3664, F-75005 Paris, France. ${ }^{17}$ Sorbonne Université, Institut Curie, CNRS, UMR3664, F-75005 Paris, France. ${ }^{18}$ NanoBioMedical Centre, Faculty of Physics, Adam Mickiewicz University, Umultowska 85, 61-614 Poznan, Poland. ${ }^{19}$ Department of Biology, Tufts University, Medford, MA USA. ${ }^{20}$ Ludwig Institute for Cancer Research, University of California School of Medicine, San Diego, La Jolla, CA, USA. ${ }^{21}$ Department of Medicine, University of California School of Medicine, San Diego, La Jolla, CA, USA. 22 Institut Curie, PSL Research University, CNRS, UMR3348 F-91405, Orsay, France. ${ }^{23}$ University Paris Sud, Paris-Saclay University, CNRS, UMR3348, F-91405, Orsay, France. ${ }^{24}$ Genome Integrity and Structural Biology Laboratory, National Institute of Environmental Health Sciences, Durham, NC, USA. ${ }^{25}$ Department of Biochemistry, University of Oxford, South Parks Road, Oxford, OX1 3QU, UK. ${ }^{26}$ Department of Molecular Genetics and Microbiology and University Program in Genetics and Genomics, Duke University Medical Center, Durham, NC USA. ${ }^{27}$ Department of Environmental and Radiological Health Sciences, Colorado State University, Fort Collins, CO, USA. ${ }^{28}$ Program in Genetics, Tufts University, Boston, MA, USA. ${ }^{29}$ Department of Biology and Rosenstiel Basic Medical Sciences Research Center Brandeis University, Waltham, MA, USA. ${ }^{30}$ Department of Molecular Genetics and Microbiology, Duke University Medical Center, Durham, NC USA. ${ }^{31}$ Department of Cellular and Molecular Medicine, University of California School of Medicine, San Diego, La Jolla, CA, USA. ${ }^{32}$ Moores-UCSD Cancer Center, University of California School of Medicine, San Diego, La Jolla, CA, USA. ${ }^{33}$ Institute of Genomic Medicine, University of California School of Medicine, San Diego, La Jolla, CA, USA. ${ }^{34}$ Department of Genetics \& Development, Columbia University Irving Medical Center, New York, NY, USA. ${ }^{35}$ Department of Microbiology and Molecular Genetics, The University of Texas Health Science Center at Houston, Houston, TX, USA..
\end{abstract}

* Corresponding Authors:

Hannah Klein, New York University School of Medicine, New York, NY USA; E-mail: hannah.klein@nyumc.org;

Nayun Kim, The University of Texas Health Science Center, Houston, TX, USA; Email: Nayun.Kim@uth.tmc.edu;

Michael Lisby, University of Copenhagen, Copenhagen, Denmark; Email: mlisby@bio.ku.dk;

Anna Malkova, University of lowa, lowa City, IA, USA; Email: anna-malkova@uiowa.edu 
ABSTRACT Understanding the plasticity of genomes has been greatly aided by assays for recombination, repair and mutagenesis. These assays have been developed in microbial systems that provide the advantages of genetic and molecular reporters that can readily be manipulated. Cellular assays comprise genetic, molecular, and cytological reporters. The assays are powerful tools but each comes with its particular advantages and limitations. Here the most commonly used assays are reviewed, discussed, and presented as the guidelines for future studies. doi: 10.15698/mic2019.01.664

Received originally: 24.06 .2018

in revised form: 29.08.2018,

Accepted 14.09.2018,

Published 07.01.2019.

Keywords: chromatin dynamics, chromosome rearrangements, crossovers, DNA breaks, DNA repair centers, DNA resection, DSBs, gene amplification, gene conversion, genome instability, gross chromosome rearrangements, fluorescent proteins, Holliday junctions, homologous recombination, mitotic recombination, mutagenesis, pulsed field gel electrophoresis, R-loops, single-particle tracking, replication fork stalling, sister repetitive sequences, sister chromatid recombination, site-specific chromosome breaks, toxic recombination intermediates, yeast artificial chromosome.

\section{Abbreviations:}

5-FOA - 5-fluoro-orotic acid, ahTET anhydrotetracycline, BER - base excision repair, BIR break-induced replication, CGR - complex genome rearrangement, ChIP-seq - chromatin immunoprecipitation followed by sequencing, DSBs double-strand break, DSBR - double-strand break repair, FLIP - fluorescence loss in photobleaching, FRAP fluorescence redistribution after photobleaching, GamFP - Gam-fluorescent protein, GCR - gross chromosome rearrangement, HJs - Holliday junctions, HR homologous recombination, iFRAP - inter-foci Fluorescence Redistribution After Photobleaching, $\mathbf{L O H}$ loss of heterozygosity, MMEJ - micro-homology mediated end joining, MMS - methyl methanesulfonate, MSD - mean-square displacement, NER - nucleotide excision repair, NHEJ - nonhomologous end joining, PAFPs - photoactivable fluorescent proteins, PFGE pulsed field gel electrophoresis, RDG - RuvCDefGFP, RFBs - replication fork barriers, RTS1 - replication termination site 1, SCE - sister-chromatid exchange, SCR - sister-chromatid recombination, SDSA - synthesisdependent strand annealing, SPT - single-particle tracking, sptPALM - single-particle tracking combined with Photoactivated Localization Microscopy, SSA single strand annealing, UV - ultraviolet, YAC - yeast artificial chromosome.

\section{INTRODUCTION}

Genomes are subject to spontaneous and induced DNA damage throughout the life cycle of the host organism. DNA damage can be repaired to the original DNA sequence without a change or altered through mutagenesis and recombination events. To detect changes, genetic and molecular assays have been developed in model systems. As mutagenesis and recombination repair are rare events, reporter assays have been developed that allow for the selection of rare events. Cytological assays provide information as to real time repair events, with detection of the spatio-temporal organization of DNA and protein complexes during the repair process.

The genetic assays are based on either nutritional selection or drug/compound resistance and may be combined with physical assays of the interacting DNA molecules. Each assay is tailored to the specific type of repair event under study. Thus, it is important to bear in mind that not all repair events are captured, and that the assay is a read-out seen as a change in phenotype that may arise from more than one type of repair process. Nonetheless, this approach has proved to be very informative for the 
characterization of proteins involved and even specific mutations within these proteins that incapacitate various activities such as a nuclease or a DNA helicase or a protein interaction. The reporter assays have also been useful in parsing out the response to a particular DNA damaging agent, to determine how the DNA is damaged and repaired. These assays have also been applied to the repair pathway elucidation through the use of reporters in multiple mutant background strains. When genetic assays are combined with molecular analyses of the repair products, the combination becomes a powerful tool for the understanding of the specific repair pathway. This is further augmented with cytological studies.

Molecular assays for DNA damage and repair require that most or all of the cells have undergone the same event. Such assays can be used to examine the consequences of damage repair, such as chromosome rearrangements. Molecular assays can also be used to examine the processing and repair of induced damage, most frequently a double strand break. In these types of assays a break is induced at a specific site by placing an enzyme recognition site at a specific chromosome locus, surrounded by DNA sequences that are known and can be readily assayed by known restriction enzyme recognition sites. These assays can detect transient intermediates if sufficiently abundant, toxic intermediates and final products. Repair can be monitored in real time by PCR analysis, Southern blot studies, or pulsed field gel electrophoresis to detect large DNA molecules.

Cytological assays allow for detection of DNA lesions, DNA repair intermediates and DNA repair proteins at the single-cell level thereby providing an opportunity to deconstruct the order of events during the DNA damage response in living cells. The single cell approach can reveal cell-to-cell heterogeneity in the progression of repair processes and uncover rare events that are often lost in population-based assays. Cytological assays have also revealed global changes in nuclear organization of chromatin during DNA repair. By use of digital image analysis software, biochemical information such as diffusion rates, binding constants, and stoichiometry of repair complexes can be deduced for DNA repair proteins in their natural environment.

Here, these three areas for the study of DNA recombination and repair are presented. Although there is a similarity in some of the assays, each has been tailored to study specific repair processes. Some of the assays have been portable into mammalian cells with modification of the reporter readouts. They have proved to be powerful tools for the study of genome instability in cancer cells with defects in DNA HR genes, cell cycle checkpoint genes and non-HR repair processes. Mutations in all of these types of genes have been associated cancers and other human diseases. These guidelines should prove useful for the wider application of the current protocols and the development of additional assays. Individual author contributions and contact information are available in Supplementary Table 1.

\section{GENETIC ASSAYS TO DETECT DNA RECOMBINATION} AND REPAIR

\section{Methods to detect mutagenesis}

Forward mutation assays

Mutations negatively impacting the normal function of the gene product can be detected, provided that a practical method of counter-selection is available. In such "Forward mutation assays", the rates of mutations can be a general indicator of the repair efficiency of a given genetic background as well as the mutagenic effect of a particular treatment. In Saccharomyces cerevisiae, mutations occurring anywhere in the CAN1 or URA3 genes are detected by the resistance to the drug canavanine (Can) or 5fluoroorotic acids (5-FOA), respectively. CAN1, which encodes an arginine permease spanning the plasma membrane, has been a useful tool as a counter-selectable marker of mutagenesis since the import of the amino acid arginine as well as its toxic analog canavanine is disrupted when CAN1 gene is mutated, leading to $\mathrm{Can}^{\mathrm{R}}$ phenotype. Forward mutations in URA3 result in resistance to the drug 5-FOA, which is not toxic unless converted to 5-fluorouracil (5-FU) by the URA3-encoded orotidine-5-phosphate decarboxylase. Genetic or chemical disruption in the DNA repair pathways or the addition of the DNA damaging agents will manifest as the elevation of the rate of $\mathrm{Can}^{\mathrm{R}}$ or $5-\mathrm{FOA}^{\mathrm{R}}$ mutations. The identity of mutations analyzed by Sanger sequencing of CAN1 ORF in the Can ${ }^{R}$ mutants has led to interesting findings regarding the mutagenesis mechanism under particular conditions. For example, in cells treated with the alkylating agent methyl methanesulfonate (MMS), the mutations at the CAN1 gene largely comprised of base substitutions, most prominently $\mathrm{G}: \mathrm{C}$ to $\mathrm{T}: \mathrm{A}$ transversions [1]. Another experiment showed that the CAN1 mutation spectra shifted from comprising mostly of basesubstitutions ( $>80 \%$ ) to mostly of $2-5$ bp deletions $(>50 \%)$ when the transcription of the gene was elevated [2]. The deletion of topoisomerase I-encoding TOP1 gene led to the disappearance of the 2-5 bp deletions, demonstrating how the forward mutation assay combined with the subsequent survey of the mutation spectrum could yield critical details about the mechanism underlying a particular type of mutations. In a URA3 forward mutation assay in yeast cells expressing mutant polymerase $\delta$ or $\varepsilon$, specific hot spots of mutation were identified that serve as the mark of the error introduced by the respective mutant DNA polymerases $[3,4]$. By incorporating the URA3 gene adjacent to the early firing replication origin ARS306 in two different orientations, the sequences of the $5-\mathrm{FOA}^{\mathrm{R}}$ mutations were used to determine whether polymerase $\varepsilon$ or polymerase $\delta$ functions mainly during leading- or lagging-strand synthesis. Despite their great utility as the preliminary indicator of the mutagenicity, however, the analyses into the types of mutations occurring like those described above is generally hampered by the relatively large size of the CAN1 (1770 nt) or URA3 (801 nt). 


\section{BOX 1: GENETIC ASSAYS TO DETECT DNA RECOMBINATION AND REPAIR}

Forward mutation assays | Use of genes where loss of function recessive mutations can be selected through resistance to medium compound.

Reversion mutation assays | Restoration of function or prototrophy through reversion of a specific mutation. These assays may detect base pair changes or frameshift mutations.

Sister chromatid recombination | Assays to detect double strand break repair through exchange between sister chromatids.

Direct repeat assays | Measures gene conversion, single strand annealing and crossover recombination using heteroallelic repeats to detect rare events. Events may be spontaneous or induced by a double strand break at a cut site introduced into one of the repeats.

Recombination in diploid cells | Use of color assays for red/white colony sectoring to detect gene conversion with or without an associated crossover. This assay is often used for loss of heterozygosity (LOH) events.

Gross chromosomal rearrangements | These assays detect translocations, deletions, amplifications and chromosome fusions, all termed gross chromosomal rearrangements. The basic design of the assays use multiple counterselectable markers embedded in the non-essential terminal regions of chromosome arms.

Repeat expansions and genome instability | Assays to monitor repeat expansion through interference of intron function in a counterselectable gene. This assay can be adapted to many repetitive DNA sequences to determine instability.

Yeast artificial chromosomes and DNA sequence fragility | Insertion of simple repeat tracts in an artificial chromosome with counterselectable markers is used to monitor breakage and aberrant repair of these sequences.

Chromosome rearrangements associated with gene amplification | Use of genes that result in resistance/tolerance to cytotoxic compounds in a dosage-dependent fashion. This type of assay detects copy number variation (CNV) and can be used to detect chromosome rearrangements associated with gene amplification.

\section{Reversion mutation assays}

A mutation type of particular interest at a defined location can be detected by purposely designed reversion mutation assays. In mutagenesis assays where an in-frame stop codon is inserted into the open reading frame (ORF) of a selective marker gene, a range of base-substitutions negating the stop codon can be detected by the phenotypic reversion. LYS2 gene encodes an alpha aminoadipate reductase, essential for the lysine biosynthesis. Mutations at the TAA stop codon inserted into the LYS2 ORF- other than TAA to TGA or TAG - results in the selectable Lys+ phenotype. While the rate of Lys+ mutation at the Iys2-TAA allele can be a measure of the DNA damage and/or repair efficiency, the types of mutations can provide further information into how these mutations occur. Although TAA to GAA or TCA mutations are prevalent when the base excision repair pathway is disrupted, these mutations are drastically reduced upon the deletion of translesion polymeraseencoding genes REV1 or REV3 [5]. Further, the study showed that the dCMP-transferase activity of Rev1 is critical in the T:A to $\mathrm{G}: \mathrm{C}$ transversion mutations. Another example of a reversion assay is the trp5 assay. A screen for the trp- auxotroph identified the Glu-50 residue of the Trp5 protein to be essential for its function in tryptophan biosynthesis [6]. Starting with the trp5 alleles with NAA or GNA at the codon 50 (nt position 148, 149, and 150), the rate of true reversion mutations, restoring the GAA codon, are identified by selecting for Trp+ cells. Starting with six trp5 mutant strains (A148, C148, T148, C149, G149, T149), this assay allows the comparative analysis of the rate of six different base substitutions (e.g. A148G (AAA to GAA) and G149A (GGA to GAA). Using this assay, the mutation signatures of UV and 5-AZ were identified as $G$ to $A$ and $C$ to $G$, respectively.

Versatile, frameshift reversion assays to detect frameshift mutations

The sequence of a gene contains three alternative reading frames that reflect the triplet structure of codons. Only one of these frames encodes the functional product and is designated as the ORF; the other two frames specify different amino acids and are punctuated by frequent stop codons. A frameshift mutation is defined as a net addition or deletion of base pairs (bp) in a non-multiple of three $(+1$ and -1 frameshifts, respectively), which "shifts" the ORF into one of the two alternative reading frames. After the shift, a translating ribosome adds incorrect amino acids to the growing peptide until the first stop codon is encountered and this almost invariably gives rise to a nonfunctional product. Reversion of a frameshift mutation can occur by reversal of the original mutation or through acquisition of a compensatory frameshift mutation of net opposite sign. The compensatory frameshift is constrained to occur in a theoretical window defined by the most proximal stop codons in the alternative reading frames (Figure 1A). A compensatory mutation downstream (promoter 
distal) of a frameshift mutation, for example, must occur before the first stop codon is encountered in the shifted reading frame. Upstream of the frameshift mutation, the compensatory change must not occur so far upstream that it leads to the encounter of a stop codon in the other, alternative frame. The region between the original and compensatory frameshifts specifies incorrect amino acids that may inactivate the encoded protein, and this can further restrict the theoretical reversion window.

The yeast LYS2 gene is essential for lysine production, and mutants fail to grow on minimal medium lacking lysine. Selection for growth of mutants allows the ready identification of Lys ${ }^{+}$revertants, and forward mutations in LYS2 can additionally be identified by their ability to grow in the presence of the toxic compound $\alpha$-aminoadipate [7]. The LYS2 ORF is large by yeast standards ( $4.2 \mathrm{~kb})$ and its utility in frameshift reversion assays was discovered following the filling in of a unique $\mathrm{Bg} / \mathrm{ll}$ restriction site located $\sim 390 \mathrm{nt}$ from the start codon [8]. This adds $4 \mathrm{bp}$ to the sequence and creates a net +1 frameshift mutation. Whereas most frameshift mutations fail to revert in the absence of an added mutagen, the resulting $l y s 2 \Delta B g l$ allele reverts spontaneously at a rate of $\sim 2 \times 10^{-9}$. The theoretical reversion window where a compensatory, net -1 frameshift can occur is $\sim 150 \mathrm{bp}$ and is highlighted gray in Figure 1B. The Bg/ll site is indicated in yellow and the ORF is reading frame 1 ; the addition of $4 \mathrm{bp}$ shifts translation to reading frame 3 . A compensatory frameshift downstream of the filled in Bg/lI site must occur before the first stop codon (boxed) in reading frame 3 is encountered and this delimits the distal end of the reversion window. In the other alternative reading frame (frame 2), an upstream compensatory frameshift will terminate translation if it occurs before the first stop codon upstream of the engineered frameshift. In early studies, compensatory, net -1 frameshift mutations were scattered throughout the window, suggesting tolerance to most if not all amino acid substitutions in the corresponding region of the Lys2 protein. After characterization of lys $2 \triangle B g$ l revertants, an analogous -1 frameshift allele (lys2 $\triangle A 746)$ was constructed in the same region, allowing
A.
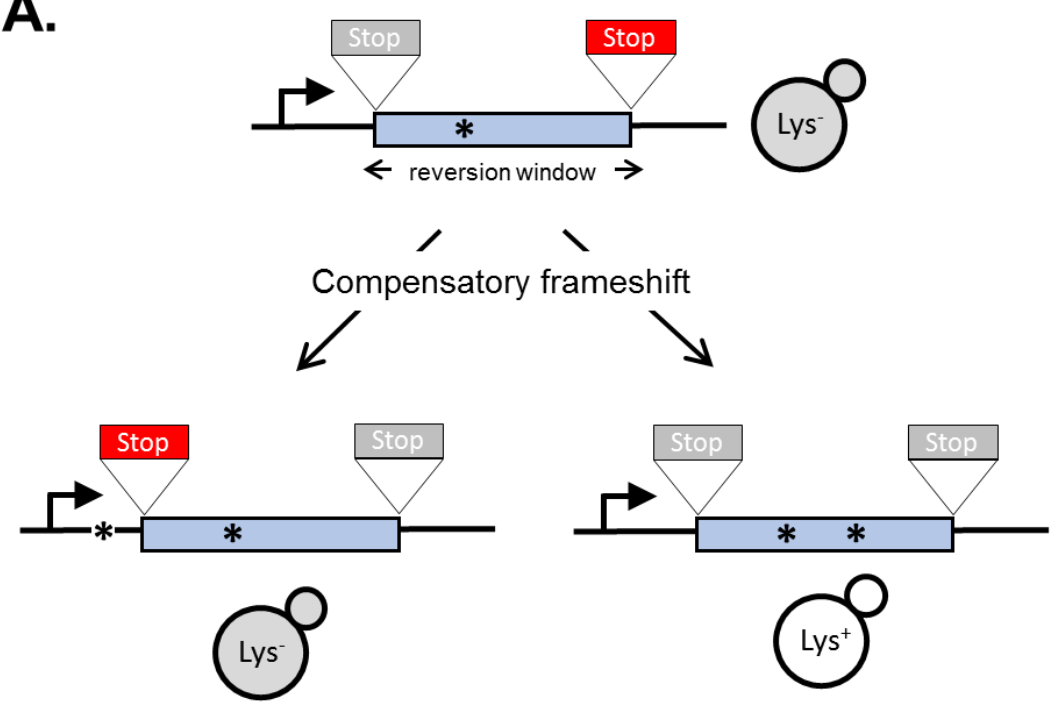

B.

CTAGCTGAAAAAATTCAAAGTTGCCAAGATCTGGAAAGGACCCCTCAGTTGTTCCGTTTG

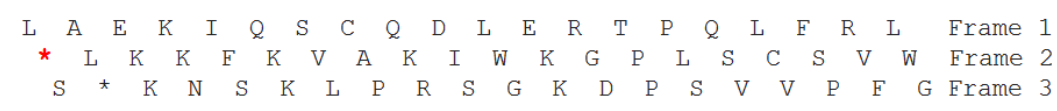

\section{GCCTTTTTGGAAAACCAAGATTTCAAATTAGACGAGTTCAAGCATCATTTAGTGGACTTT}

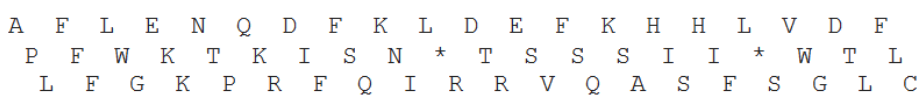

\section{GCTTTGAATTTGGATACCAGTAATAATGCGCATGTTTTGAACTTAATTTATAACAGCTTA}

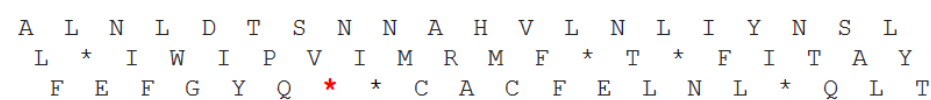

FIGURE 1: LYS2-based frameshift reversion assays. (A) Cartoon illustrating how stop codons in alternative reading frames define a reversion window (blue). The horizontal arrow indicates the direction of transcription and asterisks correspond to frameshift mutations. A stop codon encountered during translation is red; a gray stop codon is in a different reading frame and does not affect translation. (B) Sequence of the region of the LYS2 ORF that contains the lys $2 \triangle B g l$ reversion window (gray), which is delimited by the boxed stop codons. Mononucleotide runs $>3 \mathrm{~N}$ are underlined and the $B g / l l$ site is highlighted yellow. 
the isolation and characterization of net +1 compensatory frameshifts [9]. More recently, it was found that large deletions that remove the frameshift mutation and generate an in-frame fusion protein produce a functional Lys 2 protein, revealing that the first $\sim 700 \mathrm{bp}$ of the ORF are functionally dispensable (J.E. Cho and S. Jinks-Robertson, unpublished). In addition, the insertion of at least $1 \mathrm{~kb}$ of in-frame exogenous sequence into the reversion window is tolerated (Y.F. Hum and S. Jinks-Robertson, unpublished).

The lys 2 frameshift-reversion assays have been used to study a variety of DNA-metabolic processes. Studies typically involve the measurement of reversion rates coupled with sequencing of the reversion window to determine mutation patterns and rates of specific of frameshift types. Early studies with the lys $2 \triangle B g l$ and lys $2 \triangle A 746$ alleles revealed, for example, that the most common mutations were deletions and additions of a single bp, respectively. Most occurred due to DNA polymerase slippage in mononucleotide runs $>3 \mathrm{~N}$ (underlined in Figure 1B), and these "hotspots" were further amplified upon disruption of the post -replicative mismatch repair machinery $[8,9]$. To further examine properties of DNA polymerase slippage and subsequent mismatch repair, $10 \mathrm{~N}$ runs that were either out-of-frame or in-frame were inserted into the reversion window, with the latter being used to identify forward mutations that alter the length of the engineered run [10, 11]. In addition to examining frameshift mutagenesis that occurs in the context of replicative DNA synthesis, the lys $2 \triangle A 746$ assay has been used to study the genetic regulation of DNA damage bypass by Pol $\zeta$, an error-prone translesion synthesis DNA polymerase [12]. DNA damage that persists in the absence of the nucleotide excision repair pathway, for example, is associated with appearance of distinctive mutation hotspots in which the selected +1 frameshift mutation is associated with one or more base substitutions. These novel events require Pol $\zeta$ activity [13] Given the bias for frameshift mutations to occur in mononucleotide runs, these runs were eliminated in order to study other potential types of frameshifts. De novo duplications not detected previously became prominent and were shown to require the non-homologous end-joining pathway that is used to repair double-strand breaks [14]. Finally, the lys2 frameshift reversion assays have been the platform for assessing the effects of high levels of transcription on stability of the underlying DNA template [15]. This has enabled the study of templated mutations that occur at quasi-palindromes [16] and has been particularly useful for studying a 2-bp deletion signature that reflects Top1 activity [17]. Finally, the functional constraints on sequence within the reversion window has allowed for the introduction of out-of-frames cleavage sites for megaendonucleases and the study of their subsequent repair by error-prone nonhomologous end-joining (S. Shaltz and S. Jinks-Robertson, unpublished) or homologous recombination [18].

The dispensability of the amino terminus of the LYS2 gene has permitted the construction/insertion of defined types of frameshift alleles and the study of the diverse mechanisms that revert them. Although small changes are limited to the reversion window, this window could be expanded to at least a kb by inserting a synthetic fragment that lacks stop codons in all three reading frames. In addition, the ability of the Lys 2 protein to function without its amino terminus allows large deletions that create functional fusion proteins to be identified. Although the description here has been limited to the LYS2 gene, the same principles can be applied to any gene with a similarly dispensable region or to $\mathrm{N}$-terminal fusion proteins.

\section{Methods to detect mitotic recombination}

DNA breaks are among the most harmful lesions; they block transcription and replication causing cell lethality unless properly repaired. Double strand breaks (DSBs) can be repaired by different mechanisms, from nonhomologous (NHEJ) and Micro-homology-mediated End Joining (MMEJ) to homologous recombination (HR). HR is the most prominent error-free mechanism of DSB repair. It relies on the interchange of information between two homologous DNA sequences and on the copying of information from a homologous template to seal the break [19, 20]. DSBs occurring in the S-G2 period of the cell cycle are preferentially repaired by $\mathrm{HR}$, when the sister chromatid can be used as a template in an error-free manner to maintain the stability of the genome [21-23].

Homologous recombination has been studied both in mitosis and meiosis since the beginning of the century in Drosophila, bacteria, yeast, filamentous fungi and in superior organisms using genetic assays. The detection of mitotic recombination originally relied on crosses between strains carrying heterozygous markers that allows to distinguish phenotypically the recombinant products from the parental configuration. Thus, mitotic recombination was studied between homologous chromosomes in eukaryotic diploids or prokaryotic merodiploids. The capacity to artificially engineer genomes allowed to generate intrachromosomal systems for the detection of $H R$ that fostered the studies on mitotic recombination [24, 25, 26]. All systems for detection of recombination developed in the last three decades have proved to be tremendously effective to decipher and expand our knowledge on the different mechanisms of HR, including Single-Strand Annealing, SynthesisDependent Strand Annealing, Gene Conversion, Crossovers, etc. $[27,28]$. Many different assays have been developed for the genetic detection of recombination. Along the years, a number of them have been developed, both in plasmids and integrated in chromosomes for the analysis of different mitotic recombination events and mechanisms. A detailed description of them together with the methodology of use has been published [29].

\section{Method to detect Sister Chromatid Recombination}

Provided that spontaneous breaks arise commonly during and after replication, and that the sister chromatid is the preferred template for HR, Sister Chromatid Recombination (SCR) can be considered the major mechanism for HR repair. It is, therefore, important to decipher whether SCR uses the same HR factors and mechanisms than recombi- 
nation between homologous chromosomes as well as to determine which are the factors that specifically condition SCR. The first assay to study SCR in the yeast $S$. cerevisiae was a chromosomal genetic system based on two truncated repeats of the HIS3 gene in a direct orientation [30]. Recombination with the other repeat on the sister chromatid (unequal Sister-Chromatid Exchange, SCE) leads to the triplication of the HIS3 allele and allows the genetic detection of His+ recombinants [30]. This assay is not only limited to the analysis of unequal SCE but also does not allow physical detection of recombination intermediates and therefore the analysis of the kinetics of the reaction is not possible. To bypass this limitation, the two plasmid recombination systems ( $p L 2-H O r$ and pTINV) that enable the study of SCR by physical methods were developed [23, 31]. In both of them, a 24-bp mini-HO site leads preferentially to nicks in the DNA after activation of the $\mathrm{HO}$ endonuclease, which is expressed from a GAL1 promoter. HO-induced nicks are then converted to DSBs by replication [31]. The pL2-HOr system is based on a mutated copy of the LEU2 gene containing the mini-HO site [23]. Replication-born DSBs can be repaired via SCR with the equal repeat in the sister-chromatid (equal SCE) leading to DNA intermediates that can be detected physically by Southern-blot [23]. The pTINV system, by contrast, is based on two mutated repeats of the LEU2 gene, one of which contains the mini-HO site, placed in an inverted orientation. This system allows both the physical detection of SCR intermediates (arising from unequal SCE in this case) as well as the genetic detection of Leu+ recombinant products (Figure 2) [23, 31]. In order to use these plasmids, the $\mathrm{HO}$ gene under the $G A L$ promoter can be either integrated in the genome (such as in ade3::GAL-HO strains $[23,31]$ or expressed from a plasmid [32]. The strain to be studied should have the endogenous LEU2 gene deleted as well as an 'inconvertible' variant at the MAT locus (MATa-inc mutation) [33] that impedes $\mathrm{HO}$ cleavage. Importantly, although only the pL2HOr allows the detection of equal SCE intermediates, the unequal SCE gave the same results as the equal SCE, concluding that unequal SCE in the PTINV system can be used an accurate indicator of the proficiency of total SCR [23, 31, 34].

Genetically, the frequency of SCR is assayed as the frequency of Leu+ recombinants after a five hours treatment with galactose (see detailed description in [29]) (Figure 2A). Briefly, the different wild-type and mutant strains transformed with the $p T I N V$ plasmid are grown either in glycerol-lactate or raffinose-containing media to ensure glucose consumption. Doxycycline is also added to the media to prevent transcription of the leu2 repeats, which is driven by the TET promoter. Adding galactose to the media induces HO-induced expression. Spontaneous (at time 0) and $\mathrm{HO}$-induced (five hours later) recombination frequencies are calculated by performing serial dilutions and counting the number of colonies that grow in total and recombinant-selective media (lacking Leucine). The median frequency of recombination is calculated for each of the cultures and the average of at least three independent transformants is usually considered as a reliable value.
In order to analyze the appearance and kinetics of SCR intermediates at the molecular level, a time-course experiment must be performed after the galactose addition (usually $0,0.5,1,1.5,2,3,4,6$, and $24 \mathrm{~h}$ ). DNA samples from each time point are digested with $\mathrm{Xhol}$ and Spel restriction enzymes and subjected to standard southern-blot hybridization with a LEU2 specific probe (Figure 2B). At this step, the purity and specificity of the probe is essential to avoid hybridization with the endogenous genome or spurious intermediates. Upon Xhol-Spel digestion, the HOinduced DSB results in 2.4 and $1.4 \mathrm{~Kb}$ bands while unequal SCE generates a head to head dimer that produces 4.7 and $2.9 \mathrm{~Kb}$ bands. Whereas the $4.7 \mathrm{~Kb}$ band is specific for unequal SCE, a $2.9 \mathrm{~Kb}$ product can also arise as a consequence of intrachromatid recombination. Therefore, SCR levels can be calculated as the ratio between the signal at the $4.7 \mathrm{~Kb}$ band and the total plasmid DNA (the sum of the signal at all bands). When the $2.9 \mathrm{~Kb}$ is sharp enough, the frequency of intrachromatid recombination can be estimated by subtracting the signal at the $4.7 \mathrm{~Kb}$ band from the signal at the $2.9 \mathrm{~Kb}$ band. This can give a measurement of the efficiency of recombination when it does not occur with the sister chromatid in our mutant conditions. In addition, general recombination assays are used to assay for the specificity of the SCR defect detected (see for example [32] or [35]). With these assays, in addition to the role of several general HR factors in SCR [34, 36, 37], specific factors required for SCR which so far do not affect HR between ectopic sequences or homologous chromosomes have been identified, such as cohesins, HST histone acetylases, the Rrm3 helicase, the Smc5-6 complex, etc [31, 32, 35, 38].

\section{Monitoring recombination using direct repeat assays}

Recombination can occur between sister chromatids, within a chromatid, between homologous chromosomes, or between repeated sequences at different chromosomal locations, called ectopic recombination. The ability to make direct repeat recombination reporters has allowed haploid cells to be readily screened for recessive mutations that alter recombination rates $[39,40]$. While each recombination assay has its limitations, the use of direct repeat reporters has proved to be a powerful tool to determine mitotic gene conversion rates and to characterize mutations that increase or decrease gene conversion rates.

The standard reporter used for monitoring recombination between direct repeats is shown in Figure $\mathbf{2 C}$ [41]. The key features are 1) use of a nutritional selectable phenotype, here the ability to grow on medium lacking leucine, 2) different non-reverting mutations in the duplicated genes, and 3) inclusion of an additional selectable marker between the duplicated genes. We have routinely used duplications of the LEU2 gene separated by a copy of the URA3 gene with plasmid sequences adjacent to the URA3 gene. The entire construct is integrated at the LEU2 locus. To avoid interactions between the endogenous URA3 locus and the URA3 copy in the duplication, the endogenous locus allele should ideally be a deletion or if not, a nonreverting allele. The LEU2 alleles are leu2-BstEII and leu2EcoRI. Both were formed by fill-in synthesis and ligation 


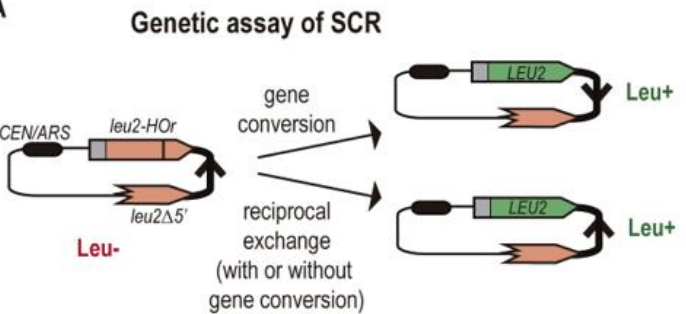

B Molecular assay of SCR

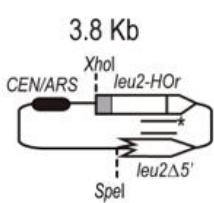

Spel
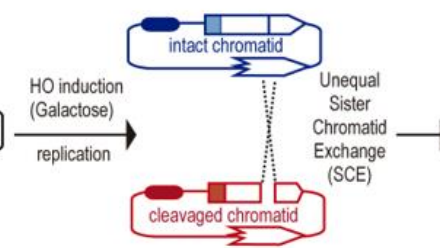
(SCE)

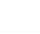

C

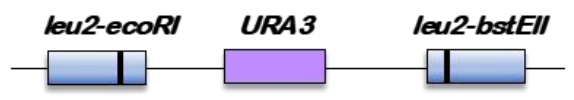

D

Gene conversion Leu+ Ura+
$4.7 \mathrm{~Kb}$

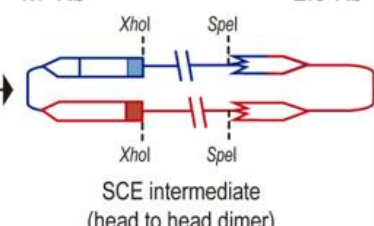

$\mathbf{E}$

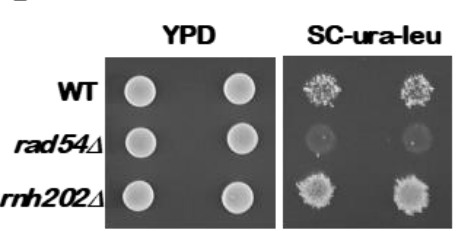

FIGURE 2: Assays to detect chromatid recombination. (A) Genetic assay of sister chromatid recombination (SCR). (B) Molecular assay of sister chromatid recombination (SCR). (C) Cartoon of the direct repeated leu2 genes with the URA3 gene inserted in between, with the black vertical line indicating the leu2 mutations. The entire reporter is integrated at the LEU2 locus and strains with the reporter have a LeuUra+ phenotype. (D) Recombination outcomes. Gene conversions are Leu+ Ura+ and retain the duplication, with one copy of the LEU2 gene now being wild type. Loss of the URA3 gene by a crossover or single strand annealing (SSA) yields Ura- segregants that can be Leu+ or Leu-. (E) Examples of Leu+ Ura+ papillae from three strains, wild type, rad54, which has very low gene conversion recombination rate, and rnh202, which has an increased gene conversion rate.

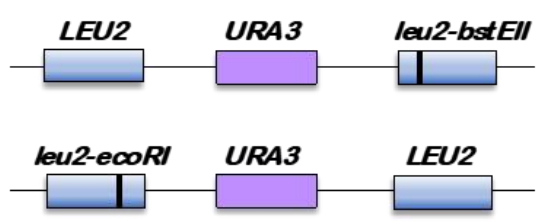

\section{Crossover or SSA Leu+ Ura- or Leu- Ura-}

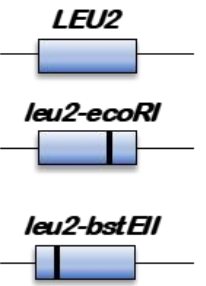

following cutting with the restriction enzymes BstEll or ECORI of a copy of LEU2 on a plasmid. The fill-in synthesis ablates the restriction site and causes a frameshift mutation in the LEU2 gene. Reversions of the restriction enzyme site mutations are not detectable, and therefore do not confound low recombination rates. The URA3 marker allows one to distinguish between various recombination events that result in a Leu+ phenotype, as shown in Figure 2D. These are gene conversion, crossovers between the two leu2 alleles, and some cases of single strand annealing (SSA). Gene conversion only events are Leu+ Ura+, while crossovers and SSA events are Ura3- but may be Leu+ or Leu- (Figure 2D). For optimal detection of gene conversion events, it is important to have the leu2 alleles separated within the LEU2 gene by several hundred nucleotides. In the example shown here, the alleles are separated by about 600 nucleotides. If the alleles are too close together, many gene conversion events will cover both alleles (called co-conversion) and will result in a Leu- phenotype. Leu+ gene conversion events result from conversion of only one of the two alleles.

Recombination rates are determined by fluctuation tests [42]. Two basic approaches can be used, the use of the median as first described by Lea and Coulson [43] or from the $p_{0}$ class, the number of cultures with no recombination events, adapted from the Luria-Delbruck fluctuation test [44] and updated by Lang and Murray [45]. Fluctuation tests are used to avoid the overweighted impact of "jackpot" events, colonies that experience a gene conversion event early in the growth of the colonies, such that a large 
proportion of cells are generated with the recombination phenotype but representing only one event. Use of the median or the poclass eliminates this issue. Rates are determined from multiple colonies of the same genotype, and from these data either standard deviations or $95 \%$ confidence limits are derived.

When doing fluctuation tests by the median method, there is a limit on the range of rates that can be detected. The wildtype rate of gene conversion using the reporter shown in Figure $2 \mathrm{C}$ is on the order of $8 \times 10^{-6}$. This can be readily detected using single colonies as the starting material. However, if rates are $10 \mathrm{X}$ lower, as occurs in homologous recombination mutants, this cannot be detected using a single colony as the starting material as a yeast colony has about $5 \times 10^{7}$ cells. Instead the colony is used to inoculate a small culture, which is then grown overnight to generate more cells. The caveat here is that plating too many cells on the selection medium is inhibitory to growth of the rare Leu+ Ura+ recombination. To avoid this, the cells must be plated on several plates, with a maximum of $10^{8}$ cells spread on one plate.

Several other caveats regarding the starting strain need to be considered. First, some mutations that reduce recombination have intrinsic increased mutation rates. Thus, it is important to first confirm that the recombination reporter is intact and gives a Leu- Ura+ phenotype. On selection medium of -leu-ura medium, a wildtype colony when replica-plated from nonselective medium will give small areas called papillations of growth (Figure 2E). A mutant with a lowered recombination rate will give few to no papillations, while a mutant with increased gene conversion will give many more papillations. Loss of the reporter as shown in Figure 2D will result in no growth or complete growth on the medium and is not informative. To avoid the accumulation of additional mutations, particularly those that result in dysfunctional mitochondria and a petite phenotype, cells should first be passaged on plates with glycerol as a carbon source. This eliminates petites that have arisen in the strain. Another concern is the potential differential growth of parental versus recombinant cells. While this most likely is not a concern for recombination assays, if it seems that the recombinant segregants have a different growth rate from the parental strain, a modified median estimator can be applied as has been for estimation of mutation rates [46]. Lastly, if recombination rates are extremely high, such that the rate is $10^{-3}$ or higher, fluctuation tests are not valid as new events cannot be distinguished from progeny of earlier events. In this case other approaches must be used that measure events within one generation [47]. However, this is not a significant concern for gene conversion assays.

\section{Ade2-based colony color sectoring assays to detect mitotic recombination}

Spontaneous mitotic recombination can be detected between chromosome homologs in diploids, or between artificial duplications in haploid or diploid cells. Because spontaneous mitotic recombination occurs at low frequencies, a selection step is generally required to detect it. In most assays, the two recombining sequences contain two different mutant alleles (heteroalleles) of a selectable gene to allow detection of rare recombination events during growth of a culture [48]. To provide a visual assay for mitotic recombination, several recombination reporters based on the ADE2 gene have been developed [49-52]. Yeast ade 2 mutants accumulate a red pigment, resulting in red colonies, whereas cells with functional $A D E 2$ form white colonies. Diploid cells with ade2 heteroalleles, or haploids with artificial ade 2 repeats, form mostly red colonies with rare recombination events detected as white $\mathrm{Ade}^{+}$sectors or papillae (Figure $\mathbf{3 A}$ ). The colony-sectoring phenotype provides a qualitative read out and has been useful to identify mutants with altered rates of recombination $[50,53,54]$. A fluctuation test can be performed to measure the rate of $\mathrm{Ade}^{+}$recombinants within a culture.

The design of the assay consists of two recombination reporters with ade2 heteroalleles placed in direct or inverted orientation on the same chromosome (Figures 3B and 3C) [50-52]. Recombination can occur intrachromosomally or between repeats of misaligned sister chromatids. For direct repeats, gene conversion events retain the intervening marker, while a crossover between repeats results in loss of the marker and one of the repeats. Deletion of one of the repeats and intervening sequence can also occur by single-strand annealing (SSA), a Rad51independent mechanism. The inverted-repeat substrate was originally designed to avoid the contribution of SSA to recombination events. Conversion of ade2- $n$ by the ade2 $5^{\prime} \triangle$ allele restores $A D E 2$. Around $50 \%$ of $\mathrm{Ade}^{+}$recombinants exhibit inversion of the TRP1 gene located between the heteroalleles; these events could occur by a crossover or long tract conversion between misaligned sisterchromatids $[55,56]$. One of the challenges with using the ade2 reporters is that the red pigment is slightly toxic; therefore, $\mathrm{Ade}^{+}$recombinants have a growth advantage. To avoid this problem, it is important that colonies are picked after only three days growth on rich medium to perform fluctuation tests; if grown for longer, the apparent rate of recombination increases.

To facilitate analysis of unselected recombination events, a version of the direct repeat recombination reporter was generated with an I-Scel cut site inserted in one of the ade 2 repeats (Figure 3B) [51]. In these strains, I-Scel nuclease is expressed from a galactose-inducible promoter. The frequency of recombination is measured by the number of colonies that grow on medium containing galactose (I-Scel constitutively expressed) compared with the number on glucose-containing medium (I-Scel off). Most of the colonies that survive I-Scel expression are recombinants because imprecise NHEJ to eliminate the I-Scel site is very rare in yeast. Although most of the DSB-induced recombinants recovered on non-selective medium are $\mathrm{Ade}^{+}$, some are Ade- due to copying the ade2- $n$ mutation from the donor allele during recombination. Gene conversion products can also be detected in real time by Southern blot of genomic DNA digested with appropriate restriction enzymes [57]. 
A

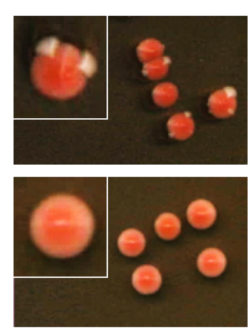

B

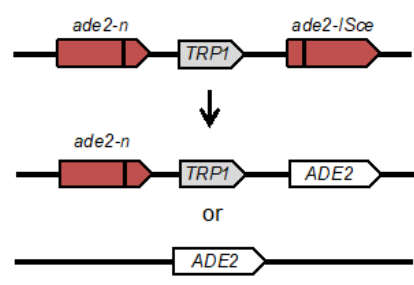

C

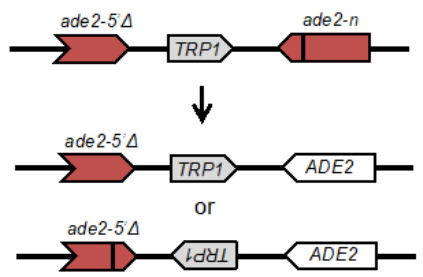

D

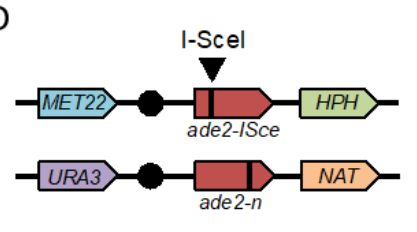

E

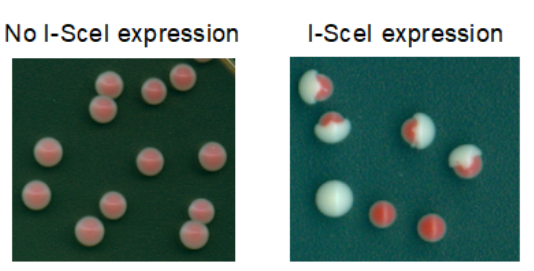

$\mathrm{F}$

I-Scel induced DSBs in $\mathrm{G} 2$ cells

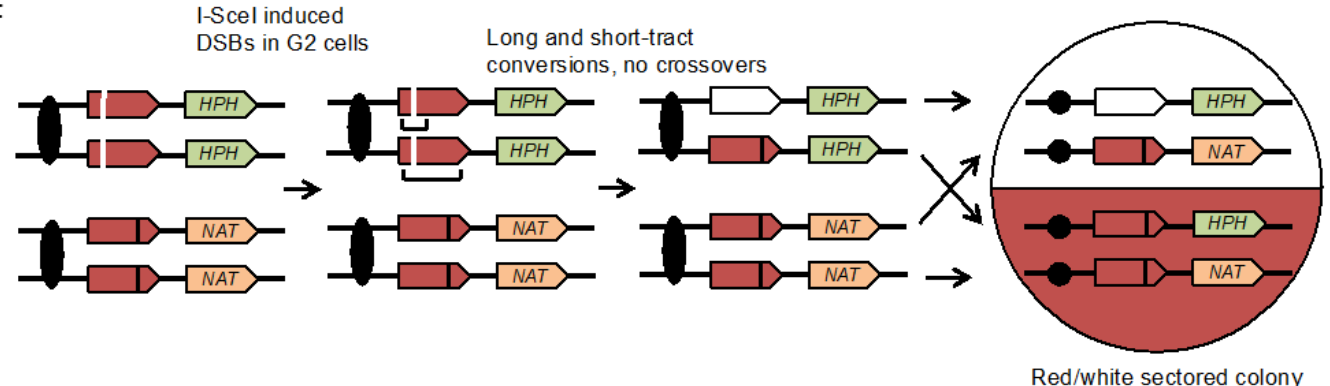

Long and short-tract

G

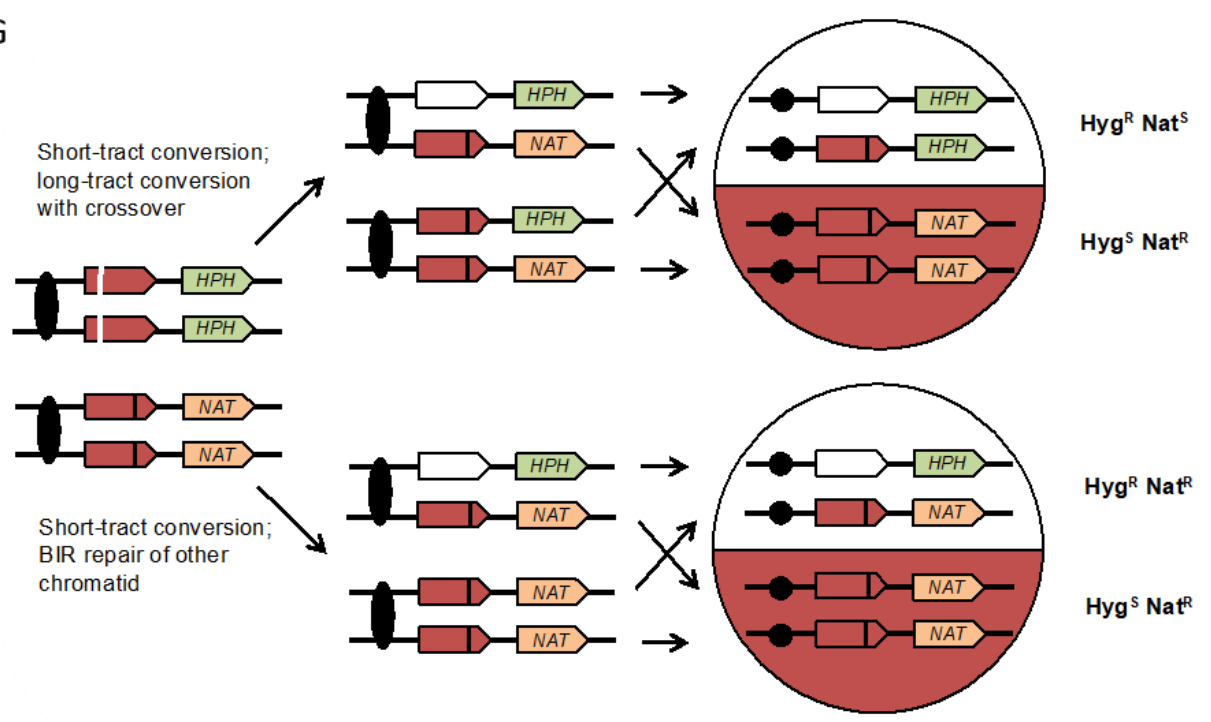

FIGURE 3: Colony color sectoring assays to detect spontaneous or DSB-induced recombination in haploid and diploids cells. (A) The upper panel shows wild-type colonies with the inverted-repeat reporter; rad52A (recombination-deficient) colonies are shown in the lower panel. (B) Direct-repeat recombination reporter: the ade2- $n$ allele was generated by restriction enzyme fill-in of a Ndel site, the ade2-ISce allele was made by inserting the I-Scel cut site at the Aatll site. Gene conversion events retain the TRP1 marker, whereas crossovers (CO) or SSA result in loss of TRP1. (C) Inverted-repeat recombination reporter: the ade2- $n$ and ade2-5' $\triangle$ alleles are place in inverted orientation and separated by TRP1. Gene conversion events retain TRP1 in the original configuration, whereas CO or long-tract sister-chromatid conversion flips the orientation of TRP1. (D) Diploid with ade2- $n$ and ade2-ISce heteroalleles, heterozygous markers $150 \mathrm{~kb}$ downstream of the ade2 loci and heterozygous markers on the other chromosome arm. (E) Examples of colonies before and after I-Scel induction. (F) I-Scel cuts both chromatids with the ade2-ISce allele in G2 cells and repair occurs by short or long tract conversion yielding ADE2 or ade2- $n$ allele, respectively. If both conversion events are non-CO, the colony is white/red sectored and the two halves retain heterozygosity for $H P H$ (hygromycin resistance) and NAT (nourseothricin resistance). (G) If repair of one chromatid is associated with a CO and the recombinant chromatids segregate to different daughter cells, reciprocal $\mathrm{LOH}$ is detected. Note that if the recombinant chromatids segregate to the same daughter cell at mitosis, the CO is not detected and this must be taken into account when calculating the frequency of CO. BIR results in a half sector that retains heterozygosity and the other has LOH of the HPH marker. 
Ade2-based assays to detect recombination between heteroalleles in diploid cells

The rate of spontaneous recombination between heteroalleles on chromosome homologs of diploid cells is $\sim 40$-fold lower than observed for heteroalleles oriented as direct repeats in haploids [51]. Recombination between heteroalleles in diploid cells can occur by gene conversion with or without an associated crossover. Use of diploids with heterozygous markers on opposite chromosome arms allows detection of crossovers by loss of heterozygosity (LOH) for the marker centromere distal to the heteroalleles. However, LOH can also occur by break-induced replication and these events can only be distinguished from crossovers if both products of the daughter cells from the recombination event are recovered. To facilitate such analysis, an IScel site was incorporated into one chromosome homolog to enable analysis of unselected recombination events (Figure 3D) [49]. Induction of I-Scel results in a large increase in white and red/white sectored colonies (Figure 3E). $\mathrm{LOH}$ events can be detected by replica plating colonies to medium containing hygromycin or nourseothricin. Crossovers are detected by one sector that is $\mathrm{Hyg}^{\mathrm{R}} \mathrm{Nat}^{S}$ while the other is $\mathrm{Hyg}^{\mathrm{S}} \mathrm{Nat}^{\mathrm{R}}$ (Figure 3F). BIR results in colonies in which one sector shows $\mathrm{LOH}$ for the $\mathrm{HPH}$ marker and the other retains heterozygosity (Figure $\mathbf{3 G}$ ). If both broken chromatids repair by a crossover, the event cannot be distinguished from one with no crossovers. Colonies or half sectors that fail to repair a broken chromatid are detected by simultaneous loss of the MET22 and HPH markers. The diploid assay shown can also be used to measure spontaneous (no I-Scel induction) LOH by selection for colonies that grow on 5-FOA-containing medium [49]. Events that are Ura $\mathrm{Hyg}^{\mathrm{S}} \mathrm{Nat}^{\mathrm{R}}$ are due to chromosome loss, whereas $\mathrm{Ura}^{-} \mathrm{Hyg}^{\mathrm{R}} \mathrm{Nat}^{\mathrm{R}}$ events result from mitotic recombination.

\section{Detection and analysis of mitotic recombination events}

There have been three major challenges for the development of genetic assays of mitotic exchange: 1 ) if recombination events are resolved in $G_{2}$ of the cell cycle, two daughter cells with recombinant products will be generated, and most selective methods detect only one of these products [58]; 2) the rate of spontaneous mitotic recombination events is four to five orders of magnitude less than for meiotic recombination [59], requiring selective or very efficient screening methods for detection; 3) most studies have been limited to analyzing a single genetic locus rather than a more global analysis of recombination events throughout the genome.

A selectable red/white sectoring system that largely overcame some of these challenges [59] is shown in Figure 4A. The starting diploid is homozygous for the ade2-1 allele. This allele has a nonsense mutation at codon 65 that can be partially suppressed by the tRNA suppressor encoded by SUP4-O. In the absence of the suppressor, strains with the ade2-1 mutation accumulate a red pigment, resulting in a red colony. A diploid with a single copy of SUP4-o results in partial suppression, producing a pink colony. If a crossover occurs between the centromere and the heterozygous SUP4-o marker and if the two recombined chromatids seg- regate into different daughter cells, one daughter cell will receive zero copies of SUP4-O (producing a red colony or a red sector) and the other daughter will receive two SUP4-O copies (producing a white colony or a white sector). Thus, if the crossover event occurs at the first cell division after plating, a red/white sectored colony will be formed. The two reciprocal products of the crossover can be purified from the two sectors. The system shown in Figure 4A, therefore, solves the first challenge described above. It should be noted that this system detects only half of the crossovers, since segregation of the two recombined chromatids into one cell and two unrecombined chromatids into the other, does not produce a sectored colony. The two different types of segregation are approximately equally frequent [60].

The system described above is a screen for recombination events rather than a selection. A modification of this system allows for selection of crossovers [59]. A diploid was constructed in which one copy of CAN1 (a gene located near the left end of chromosome V) contained the can1100 allele, an ochre-suppressible mutation. The other copy of CAN1 was deleted and replaced by SUP4-O. Strains with a functional CAN1 gene are sensitive to the arginine analog canavanine. In the diploid shown in Figure 4B, the strain is sensitive to canavanine because the can1-100 allele is suppressed by SUP4-O. In addition, as in the strain shown in Figure $4 A$, the diploid forms pink colonies because of the partial suppression of ade2-1. A crossover between the SUP4-o/can1-100 markers and the centromere of chromosome $\mathrm{V}$, followed by the appropriate segregation pattern, results in one cell that is canavanine-resistant $\left(\mathrm{Can}^{\mathrm{R}}\right)$ because it lacks the SUP4-o suppressor and one cell that is $\mathrm{Can}^{\mathrm{R}}$ because it lacks the suppressible can1-100 allele. Thus, mitotic crossovers are selected as $\operatorname{Can}^{\mathrm{R}}$ red/white sectors (Figure 4B). This system can select for events that occur at a rate of $<10^{-6} /$ cell division.

Although the systems shown in Figures $4 A$ and $4 B$ allow one to estimate the rate of crossovers between the can1100/SUP4-o marker and the centromere, the location of the crossover within this interval is not determined. The system was slightly modified by constructing the diploid by mating haploid strains with about $0.4 \%$ sequence divergence (Figure 4C) [61-63]. In the resulting diploid, singlenucleotide polymorphisms (SNPs) occur at an average distance of $<1 \mathrm{~kb}$. In such a strain, when genomic samples are prepared from each side of a sectored colony, they can be analyzed by SNP-specific microarrays. In these arrays, each SNP is represented by four 25-base oligonucleotides, two with Watson and Crick strands of one allele and two with Watson and Crick strands of the second allele. Genomic samples heterozygous for a particular SNP hybridize about equally well to all four oligonucleotides, whereas samples with homozygous for one SNP hybridize better to one pair of oligonucleotides than the other. With the appropriate control DNA samples, such arrays can readily distinguish whether the sector is heterozygous or homozygous for each SNP. The position of LOH identifies the position of the crossover [62]. This type of analysis can be performed on sectored colonies to examine events on a single chromo- 
some arm or, in strains with elevated levels of recombination events, throughout the genome. The pattern of crossovers and associated gene conversion events detected in such experiments can be revealing about the mechanisms of spontaneous and induced mitotic recombination [63-66] Although many of the earlier experiments were done using SNP-specific microarrays, sequencing of genomic DNA isolated from the sectored colonies allows mapping of recombination events to even greater resolution.

Originally, the experiments were done on the left arm of chromosome V. Since this arm is relatively short (about $150 \mathrm{~kb}$ ), it has a low rate of crossovers and a selective system for detection of the events is essential. It should be noted, however, that the selection system does not function well for every chromosome location. When the can1100 and SUP4-o genes were inserted near the right telomere of chromosome IV, the diploid had only partial sensi- tivity to canavanine [63], preventing an accurate measurement of the rate of $\mathrm{Can}^{\mathrm{R}}$ red/white sectors. The red/white sectoring system, however, allowed nonselective screening for mitotic crossovers which occurred at a frequency of about $6 \times 10^{-5} /$ division. There are several advantages to using the non-selective red/white sectoring assay on chromosome IV. First, the right arm of IV contains about $1 \mathrm{Mb}$ of DNA, roughly $10 \%$ of the yeast genome, whereas the left arm of chromosome $\mathrm{V}$ represents only $1 \%$ of the genome. Second, the non-selective method allows detection of recombination events that are non-reciprocal. For example, a break-induced replication (BIR) event on chromosome IV would produce a red/pink sectored colony instead of a red/white colony (Figure 4D). Such a colony would not be detectable by the selective method shown here. Third, unrepaired DNA lesions that stimulate recombination in the second division following cell plating can be
A.

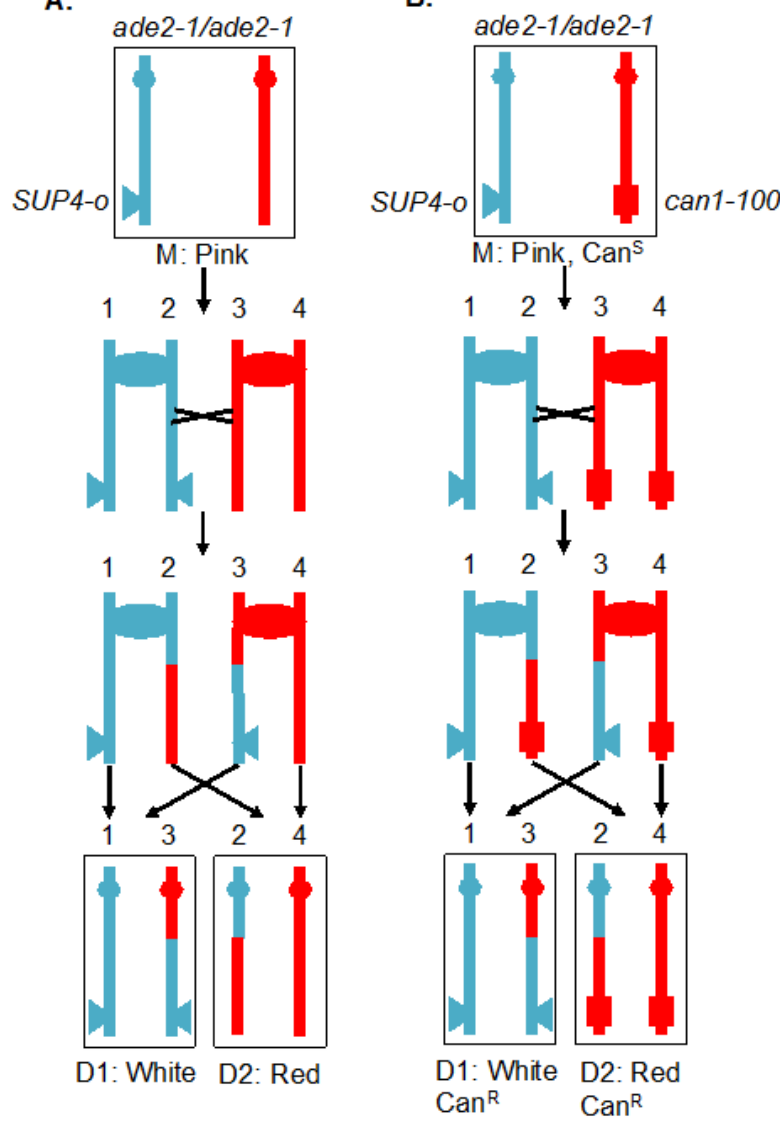

c.

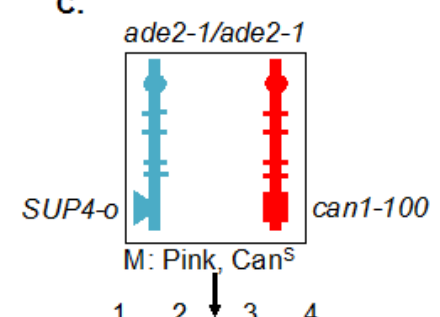

D.

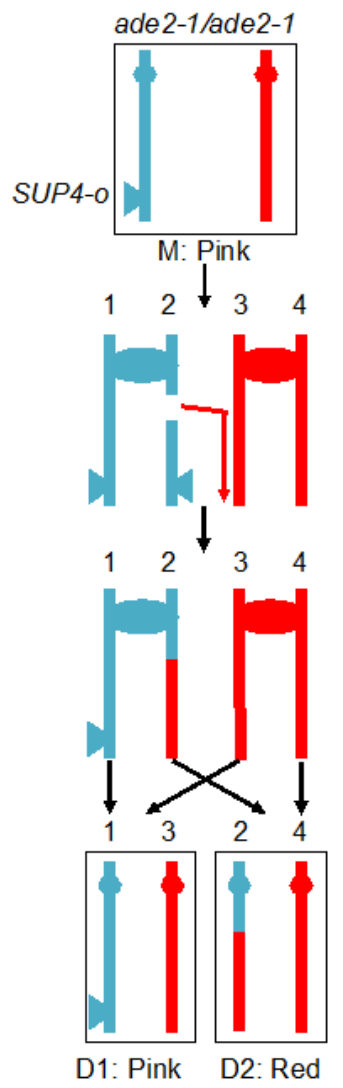

FIGURE 4: Recombination between homologous chromosomes during mitosis. Sectoring assay for monitoring mitotic crossover in the interval between the centromere (oval) and the SUP4-o insertion (triangle). Blue and red indicate the two homologs in the diploid cell. In this version of the assay, the markers are located on the left arm of chromosome $V$. (A) System for screening mitotic crossover by red/white sectoring assay. (B) Selection system of reciprocal crossovers. (C) Hybrid diploid strains with selection system for recovering reciprocal daughter cells after a crossover event, and with sequence polymorphisms (marked by blue and red ticks) for mapping positions of crossovers. (D) Detection of a non-reciprocal recombination event by a sectoring assay. In the diagram, a DSB on the chromosome containing the SUP4-o gene is repaired by a break-induced replication (BIR) event using the other homolog. The centromere-distal fragment containing the SUP4-o gene is lost, resulting in a pink/red sectored colony. 
detected by identifying a pink/white/red sectored colony [66].

The genetic systems described above have been used to map recombination events induced by gamma or UV radiation-induced recombination events $[62,65]$ and in mutants that have elevated levels of genetic instability. In the latter class are mutants that lack topoisomerase [67] or that have reduced expression of replicative DNA polymerases $[68,69]$. In principle, these methods can be used to examine many genome-destabilizing conditions.

\section{Methods to detect gross chromosomal rearrangements}

Gross chromosomal rearrangements (GCRs), including translocations, deletions, amplifications, and chromosome fusions, are believed to arise due to misrepair of DNA damage [70]. This DNA damage appears to result from cell metabolism, such as errors during DNA replication or reactive oxygen species but can also result from genetic defects that alter cell metabolism and exogenous sources of DNA damage. The rate of formation of GCRs is also influenced by features in the eukaryotic genome such as DSB-inducing sites and the presence of different types of repeated sequences; these genomic features also influence the structures of the GCRs that are formed (reviewed in [70]). Multiple pathways suppress the formation of GCRs, including pathways that are relatively specific for suppressing GCRs that result from different genomic features such as duplicated sequences. Stable GCRs are known to underlie a variety of genetic diseases [71], and the presence of GCRs and ongoing formation of GCRs are a characteristic feature of many types of cancers [72-74]. Moreover, mutations in a number of the genes encoding proteins that act in suppressing the formation of GCRs have been implicated as causal defects in inherited cancer susceptibility syndromes $[70,75]$.

A series of assays was developed for measuring the rates at which GCRs form in haploid S. cerevisiae strains to study the pathways that mediate the formation or suppression of GCRs [76-78]. These assays have two key features: 1) the assays are "undirected" as they do not require the formation of a specific GCR; and 2) the assays depend on loss of counter-selectable genetic markers present in non-essential terminal regions of chromosome arms, such as the left arm of chromosome $\mathrm{V}$ from PCM1 to the telomere. The structures of three widely used GCR assay chromosomes are shown in Figure 5. A key feature of these assay chromosomes is a cassette containing two genes, URA3 and CAN1, which confer sensitivity to 5-FOA and canavanine, respectively. In the unique sequence $G C R$ (UGCR or yel068c::CAN1/URA3) assay, the cassette is inserted into a site centromeric to the DSF1-HXT13 segmental duplication, whereas in the duplicated sequence GCR (dGCR yel072w::CAN1/URA3) assay, the cassette is inserted into a site telomeric to the DSF1-HXT13 segmental duplication; the SGCR assay is the UGCR assay in which a region of short homology was inserted into the breakpoint region. The DSF1-HXT13 segmental duplication is homologous to divergent regions on chromosomes IV, X and XIV. GCRs arise at low rates during the growth of cells containing these assay chromosomes that result in the loss of the left arm of chromosome $V$ containing the URA3 CAN1 cassette and healing of the apparently broken chromosome $\mathrm{V}$ by a diversity of DNA rearrangements that restore a functional telomere on the left end of chromosome V. Cells containing these GCRs can be selected by plating cultures on media containing 5-FOA and canavanine because GCR containing cells lack the cassette and are resistant to these two toxic compounds.

GCR rates are determined by fluctuation tests [79] using the method of the median [43], which avoids the impact of "jackpot" events due to the formation of a GCR early in the growth of a culture. Because of the low GCR rates of wild-type strains, large culture volumes $(10-50 \mathrm{~mL})$ must be plated onto selective media to obtain enough GCR-containing colonies from all of the cultures analyzed in an experiment to allow calculation of wild-type GCR rates. However, the dynamic ranges of the assays are very large, allowing analysis of mutants that have greatly increased GCR rates. For example, simultaneous loss of the MEC1 and TEL1 DNA damage checkpoint genes causes a $>10,000-$ fold increase in the GCR rate, and loss of ESC2, which is involved in promoting sumoylation by the Smc5-6 complex, causes a $>30,000$-fold increase in the GCR rate [78]. The ability to analyze GCR rates has allowed the identification of many genes that function in the suppression or formation of GCRs and facilitated pathway analysis of these genes.

Because the GCR assays are undirected and can select for many different kinds of GCRs, structural characterization of the GCRs selected has provided important insights into the structure of GCRs and the mechanisms by which they are formed [70]. Multiple complementary strategies have been developed to characterize the structures of the rearranged chromosomes, including pulsed-field gel electrophoresis, PCR mapping, array comparative genomic hybridization, and multiplexed ligation-mediated primer amplification (reviewed in [70]). The adoption of whole genome sequencing (WGS), however, is rapidly supplanting these other methods, as WGS is fast and economical since many GCR-containing isolates can be multiplexed and sequenced simultaneously [79].The types of GCRs identified include terminal deletions healed by de novo telomere addition, interstitial deletions, monocentric translocations, dicentric translocations, dicentric inverted duplications (isoduplications), and dicentric chromosome end-fusions (Figure 5B; reviewed in [70]). Note that dicentric GCRs are unstable and undergo subsequent rounds of rearrangement in which the unstable dicentric GCRs are resolved into stable monocentric GCRs [70, 80]; the resulting GCRs can be quite complex.

A key feature of GCR assays is that they are strongly influenced by the chromosomal features present in the breakpoint region, which lies between the most telomeric essential gene and the most centromeric counterselectable marker gene (Figure 5A). Numerous versions of the basic GCR assay have been developed by multiple labs to probe the effect of specific chromosomal features on the formation of GCRs by placing these features in the 
breakpoint region, including duplicated sequences, inverted repeats, trinucleotide repeat-containing sequences, G-quartet containing sequences, and $\mathrm{HO}$ sites (reviewed in [70]). The best-characterized GCRs assays are the classic assay, which are the UGCR assay, the dGCR assay, and the short-sequence homology (sGCR) assay (Figure 5A) [76-78]. In general, most mutations causing increased GCR rates in the classic, UGCR, and SGCR assays also cause increased GCR rates in the dGCR assays, but the converse is not true. Hence, the dGCR assay is more useful for screening for new GCR-inducing mutations than the other GCR assays. However, the structures of the GCRs selected in the dGCR assay are dominated by translocations formed by non-allelic recombination when the assay strains are recombinationproficient [78], and thus determining the structures of these GCRs is primarily only useful in providing insights into the control of non-allelic recombination and recombination between divergent DNA sequences (Figure 5B). In contrast, a much greater diversity of types of GCRs is selected in the classic and UGCR assays, and, in particular, in the sGCR assay (Figure 5B) [77, 80-82]; this makes this group of assays more useful in determining the effects of different mutations on the formation of a much greater diversity of types of GCRs.

\section{Methods to detect genome instability induced by repeti- tive sequences}

Methods to detect the repeat expansions and the repeatmediated genome instability using a yeast artificial intron Expansions of simple tandem DNA repeats are responsible for the ever-growing number of hereditary genetic disorders in humans, such as fragile $\mathrm{X}$ syndrome, Friedreich's ataxia, Huntington's disease, myotonic dystrophy and many others $[83,84]$. A startling feature of these muta-

A.
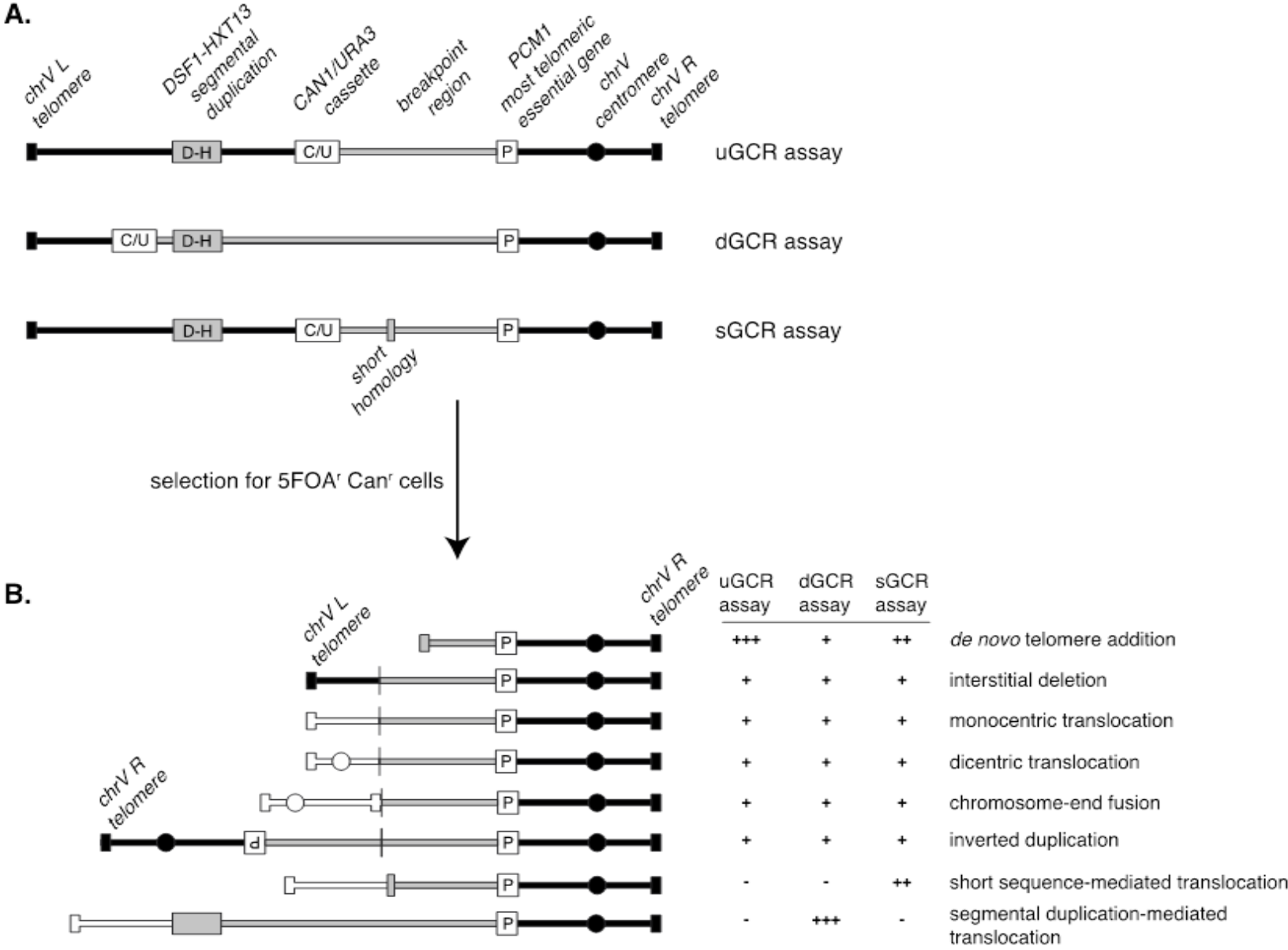

FIGURE 5. Assays to study gross chromosomal rearrangements. (A) Diagram of the uGCR, dGCR, and sGCR assays depicting the position of the CAN1/URA3 counter-selectable cassette (white box labeled "C/U") relative to the DSF1-HXT13 segmental duplication (grey box labeled " $\mathrm{D}-\mathrm{H}$ "), and the most telomeric essential gene PCM1 (white box labeled " $\mathrm{P}$ "). The grey region of the chromosome corresponds to the breakpoint region, which lies between PCM1 and the CAN1/URA3 cassette and is the region where one of the breaks associated with the GCR must occur. The grey box in the sGCR assay corresponds to a region of short homology containing a repetitive tRNA and $\sim 100 \mathrm{bp}$ of a repetitive delta sequence. (B) Selection of GCR assay-containing strains for resistance to 5-fluoroorotic acid (5-FOA) and canavanine (Can) selects for strains in which one of a number of GCRs has formed; these types of GCRs are selected at different rates in the different GCR assays. White chromosomes indicate translocations to other chromosomes (or alternatively different regions of the assay chromosomes). 
tional events is that the longer the repeat, the more unstable it is, which results in a progressively higher rate of its subsequent expansions or contractions [85]. Hence, these mutations are called dynamic DNA mutations [86], as opposed to classical static mutational changes in DNA. Studies conducted in many labs over the last two decades revealed that the instability of these repeats varies strongly depending on their sequence, length, location in the genome, ability to form alternative secondary structures, and the genetic background of the carrier cell/organism. It has also become clear that repeat expansions and other forms of repeat-mediated instability occur during practically all major DNA transactions, including replication, repair, recombination, and transcription [87-91].

The lab of S. Mirkin developed a genetically tractable system to study repeat expansions and other types of repeat-mediated genome instability in budding yeast, S. cerevisiae [92, 93]. The key component of this system is a reporter carrying an expandable repeat within an intron of the artificially split URA3 gene (Figure 6A). The reporter is linked to the TRP1 gene to allow the whole cassette to be integrated into any trp1 strain. Most studies were conducted for the Friedreich's ataxia (GAA) repeat. In this case, large-scale expansions that increase the intron's length beyond $\sim 1 \mathrm{~kb}$ inactivate the URA3 gene by blocking its splicing, this allows us to detect expansion events on a selective media containing 5-FOA [92-94]. A remarkable characteristic of this system is that the longer the repeat, the more frequently it expands further [92, 95], mimicking the genetic anticipation phenomenon known for repeat expansion diseases in human pedigrees. Another important characteristic is that repeat-mediated URA3 inactivation is a dynamic process as opposed to all-or-none mutational inactivation: the longer the expansion, the stronger the gene inactivation [92 ]. Consequently, clones with small-scale expansions are less resistant to 5-FOA than clones with large-scale expansions, resulting in smaller colonies on 5-FOA media. This makes it possible to adjust the concentration of 5-FOA to recover expansions of different scales. Besides repeat expansions, 5-FOA resistance can also result from point mutations or deletions in the body of the reporter, as well as from more complex genome rearrangements [92, 95-98]. The rates of the latter events are at least an order of magnitude higher in the presence of the $(G A A)_{n}$ repeat than in its absence.

Given this complexity, a fluctuation test is first performed to determine 5-FOA-resistance $\left(5-\mathrm{FOA}^{\mathrm{r}}\right)$ rates for the $(G A A)_{n}$ repeat in different genetic backgrounds. To distinguish between various repeat-mediated mutational events, PCR is then performed with primers flanking the repeat region, as shown in Figure 6B. Expansions are characterized by a larger PCR product. An unchanged or contracted repeat points to repeat-mediated mutagenesis, which is subsequently verified by Sanger sequencing of the reporter [95]. A lack of the PCR product is indicative of large deletions or complex genome rearrangements (CGRs), which are subsequently verified via whole genome $\mathrm{Na}$ nopore sequencing [96]. The rates of all three events: expansions, mutations and genome rearrangements, are then determined using the FluCalc program developed in the Mirkin lab, which is freely available at http://flucalc.ase.tufts.edu/ [99]. Note that to avoid statistical errors, one should carry out PCR and other analyses described above for all 5-FOA ${ }^{r}$ colonies that resulted from the fluctuation test. Practically, however, this is difficult to achieve in a fluctuation test, as the number of colonies on different 5-FOA plates can vary dramatically [44]. Thus, various approaches are employed in sampling the 5- $\mathrm{FOA}^{\mathrm{r}}$ colonies that are described in Radchenko et al. [99].

As discussed above, forward selection for the URA3 reporter inactivation is used to detect repeat expansions in our system. Note, however, that it can be easily modified to study repeat contractions via reverse selection for uracil prototrophy. An example is a modified reporter with the $(G A A)_{128}$ repeat within a longer artificial intron in the URA3 gene [100]. Splicing of the latter reporter is impaired, which makes strains carrying it fully auxotrophic for uracil. If the repeat sequence in this reporter contracts significantly, splicing is re-established, so repeat contractions are detected on media lacking uracil.

This system can also be used to study genetic instability of other DNA microsatellites, such as expandable (ATTCT) repeats [101] or yeast interstitial telomeric sequences (TGTGTGGG) $\mathrm{n}[98,102]$. These microsatellites inactivate the reporter when the length of the intron is significantly under its splicing threshold, showing that the mechanisms responsible for gene inactivation in our system differ depending on the nature of the repeat. Other mutational events, such as repeat-induced mutagenesis and CGRs were also observed for the latter repeats. Notably, studying yeast interstitial telomeric sequences using this system led to unraveling the mechanism of a very important class of chromosomal rearrangements: terminal inversions that occur during double-stranded DNA break repair via a single-strand annealing pathway [97].

Overall, the assay described here is a powerful and highly flexible and selectable system, which can be easily adopted to analyze various aspects of repeat-mediated genome instability. For example, an endogenous URA3 promoter can be replaced with the inducible GAL1 promoter to study the role of transcription [93]. The repeat itself can be inverted to study how genome instability depends on the repeat's orientation within the replication and/or transcription unit [100]. Alternatively, the whole cassette can be integrated in two orientations relative to the replication origin to study the effects of transcriptionreplication collisions [100]. Finally, the cassette can be integrated into a nonessential arm of chromosome $\mathrm{V}$ to investigate chromosomal fragility [103].

\section{Utilizing a yeast artificial chromosome to study fragility of} DNA sequences

Repetitive DNA sequences are common in eukaryotic genomes and are considered hotspots for breakage and genomic rearrangement in addition to the expansion described above. One reason that tracts of repetitive DNAs are thought to be prone to breakage is because they can form alternative structures, such hairpin loops, which can 
A

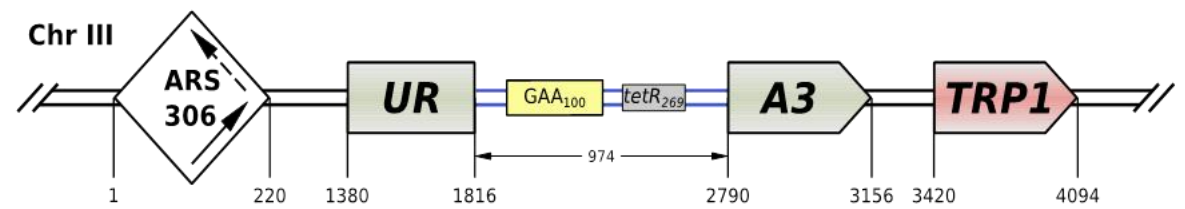

B

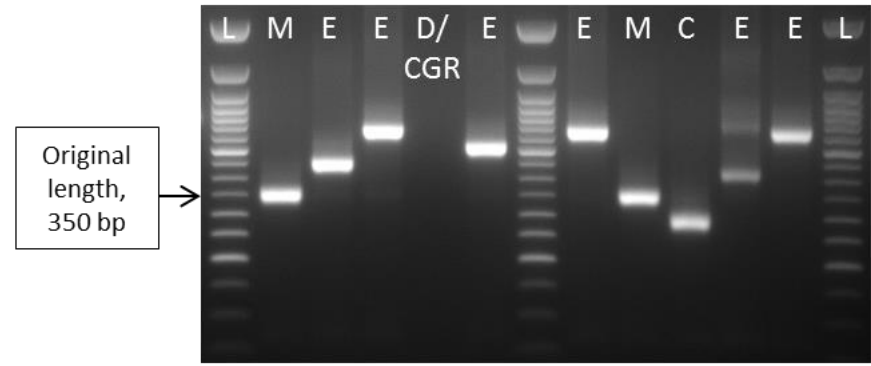

C

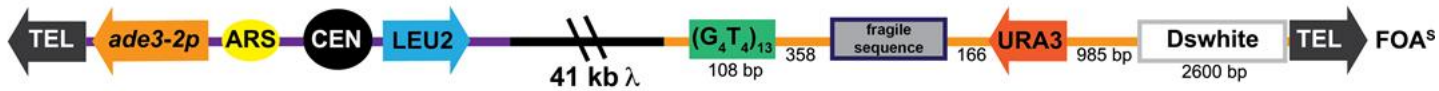

Growth that allows for breakage of fragile sequence
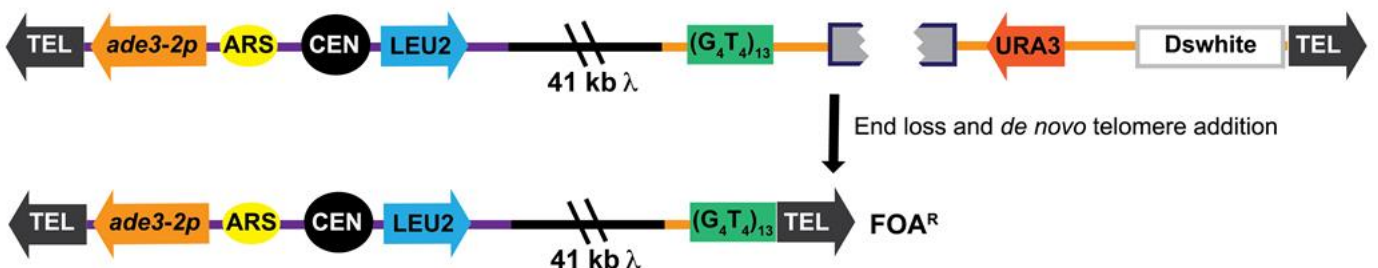

D

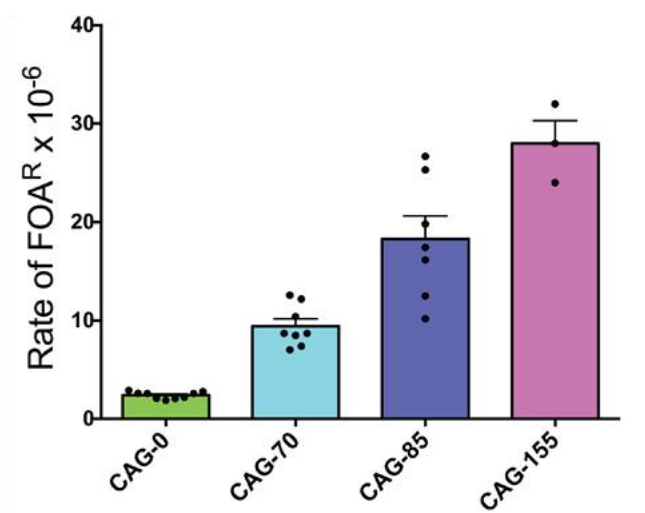

FIGURE 6: Scheme of the genetically tractable systems to study repeat expansions and repeat-mediated genome instability in yeast. (A) Scheme of the genetically tractable system to study repeat expansions and repeat-mediated genome instability in yeast. The selectable cassette contains flanking sequences from chromosome III, the URA3 gene from the pYES2 plasmid (Invitrogen) (green), an intron sequence derived from the ACT1 gene from chromosome $\mathrm{VI}$ (inside the URA3 gene) (blue), part of the tetR coding region from the pACY184 plasmid (NEB) (gray), and the TRP1 gene from the pYES3/CT plasmid (Invitrogen) (red). (B) Gel electrophoresis of the PCR analysis of 5-FOA ${ }^{r}$ colonies derived from a fluctuation test of a strain with 100 GAA repeats. $L$ - ladder, $M$ - mutation, $E$ - expansion, D/CGR - deletion or CGR (complex genomic rearrangements), $C$ - contraction with simultaneous repeat-mediated mutagenesis. The black arrow indicates a $\sim 350 \mathrm{bp}$ PCR fragment of the original (GAA) 100 repeat. (C) Assay to measure chromosome fragility. In this system, a fragile sequence has been integrated onto a yeast artificial chromosome (YAC) between a telomere seed sequence $\left(\mathrm{G}_{4} \mathrm{~T}_{4}\right)_{13}$ and the URA3 gene. Breaks that occur within the fragile sequence are subject to resection and telomere addition at the $\mathrm{G}_{4} \mathrm{~T}_{4}$ sequence, which results in loss of the URA3 gene and renders cells 5-FOA ${ }^{R}$. The YAC additionally contains a $L E U 2$ marker gene, which allows for maintenance of the YAC, a centromere (CEN4), an origin of replication (ARS1), yeast telomeres (TEL), a partially functional ade3-2 $p$ allele, and the Drosophila white gene. The orange line indicates pYIP5 plasmid backbone, the black line indicates lambda DNA, and the purple line corresponds to pUC18 plasmid backbone. (D) Wild-type fragility data of $S$. cerevisiae strain BY4705 where the fragile sequence integrated between the $\mathrm{G}_{4} \mathrm{~T}_{4}$ and URA3 marker is a CAG tract of the indicated length, in number of repeats. Assays done with this (CAG)n-URA3 YAC show that 5-FOA resistance increases with increasing number of CAG repeats. Data sourced from [107, 117, 365-367]. 
interfere with DNA replication and repair. Long tracts of CAG trinucleotide repeats are one well known type of repeat to form DNA secondary structures and are prone to expansion, contraction, and breakage. Expanded CAG repeats are responsible for over 14 genetic diseases, including Huntington's disease, myotonic dystrophy, spinocerebellar ataxias, and fragile $X$ syndrome [104].

Fragile regions break and undergo aberrant repair at a higher frequency than the average DNA sequence, making it important to understand the mechanisms that promote and protect against breakage within these DNA regions. Teasing apart the cellular mechanisms that drive chromosome fragility has been a challenge, as breakage events that occur within the genome can result in loss of essential genetic material. To understand the role of DNA sequence on chromosomal breakage, an assay was developed to measure fragility of a desired sequence in vivo in $S$. cerevisiae. The assay utilizes a non-essential yeast artificial chromosome (YAC) which was originally designed by [105].

The YAC fragility assay is an effective way to measure breakage rates of potential fragile sequences as the YAC contains little homology to any of the natural yeast chromosomes, has no essential genes, and can withstand loss of genetic material. In the structure of the initial YAC, the (CAG)n-URA3 YAC, a CAG repeat tract has been integrated between a telomere seed sequence $\left(\mathrm{G}_{4} \mathrm{~T}_{4}\right)_{13}$ and a URA3 marker gene (Figure 6C) [106]. Breakage that occurs within the CAG repeat can result in loss of the right arm of the YAC, rendering cells ura3- and resistant to 5-FOA. For YACs containing a CAG repeat, there is a length dependent increase in breakage rate as measured by 5-FOA resistance [106] (Figure 6D). The same assay has also been used for a variety of other fragile repeat sequences. For example, other derivatives of this YAC have been made that contain the CAG repeat in the opposite orientation (CTG)n [107], AT repeats that are a part of the human FRA16D common fragile site and stall replication [108], the expanded ATTCT repeat present in SCA10 patients [101], a short cruciformforming inverted repeat [109], and an H-DNA-forming repeat from the human $c-M Y C$ gene that can form triplex DNA and is found at a translocation hotspot in Burkitt lymphoma [110].

In addition to the telomere seed sequence and the URA3 reporter gene, there is also a LEU2 marker gene on the left arm of the YAC, which allows for maintenance of the YAC, a yeast origin of replication (ARS1), and a centromere (CEN4), ensuring the YAC replicates and segregates during cell division. An ade3- $p$ allele can be used to estimate the copy number of the YAC in ade2 ade3 backgrounds; an occasional non-disjunction event can create a cell with two YACs, though the existence of these few cells do not significantly affect the assay and can generally be discounted. Since there is only one yeast origin on the YAC, the direction of replication through the fragile sequence is known. For the original (CAG)n-URA3 YAC, the CAG repeat tract was integrated such that the CAG repeat is on the lagging strand template, which has been shown to be the orientation less prone to repeat tract contractions [111]. An additional design consideration is the distance between the URA3 gene and the telomere, such that the placement of the URA3 gene is far enough away from the telomere not to be subjected to telomere position effect. For this reason, a buffer sequence is included between URA3 and the telomere (the Drosophila white gene, Figure 6C) [105]. Outside of telomere position effect, point mutations in the URA3 gene could generate 5-FOA resistant colonies that have not lost the right arm of the YAC. While this is a distinct possibility for certain gene deletions, PCR of the URA3 gene from FOA resistant colonies will elucidate whether point mutation frequency is increased in a particular mutant (see [112] for an example). Alternatively, addition of a second marker gene to the end of the YAC can be utilized to eliminate events that are not due to end loss from the quantification. YACs have been constructed that contain both a URA3 and HIS3 marker [108] or both URA3 and ADE2 markers [113]. Addition of the ADE2 marker allows a visual analysis of end loss events, which will generate red $F A^{R}$ colonies on FOA-Leu plates. Other derivatives of the YAC have been made that alter the level of transcription through the CAG tract by flanking it with either transcription terminators or addition of a galactose-inducible ( $p$ Gal) promoter $[112,113]$.

A benefit of this system is the relative ease of the fragility assay. To begin, cells are plated for single colonies on yeast complete media lacking leucine and uracil (YC-LeuUra), which selects for colonies that have an intact YAC. For unstable repeats, colony PCR is used to amplify across the repetitive tract to ensure that starting colonies have the desired initial tract length. Typically, ten individual colonies (or portions of colonies) are each individually resuspended in YC-Leu liquid media. Cultures are grown for six to seven divisions which allows for breakage events to occur, and a portion of the culture is plated on media lacking leucine and containing 5-FOA. To obtain a total cell count, a portion of each culture is pooled, appropriately diluted and plated onto YC-Leu. Once colonies are counted, the rate of 5 -FOA resistance is determined by using a fluctuation analysis such as the method of the median or the maximum likelihood method to avoid over-sampling of events that occur early in the culture [114]. Occurrence of YAC end loss can be stochastic, especially for mutants that have high rates of fragility, so it is best to repeat assays at least three times to obtain a standard deviation or standard error. If desired, the structure of the healed YACs can be analyzed by Southern blot analysis or sequencing [106]. A detailed methodology of how to perform the fragility assay was recently published [115].

One important feature of the YAC fragility assay is that a method of recovering the broken chromosome is built into the design. Proximal to the fragile sequences is a 108 bp back-up $\left(G_{4} T_{4}\right)_{13}$ telomeric seed sequence from Oxytrica which has been shown to an efficient substrate for $S$. cerevisiae telomerase, but does not recombine with the natural yeast telomere sequence [116]. The $\mathrm{G}_{4} \mathrm{~T}_{4}$ seed provides an efficient pathway for healing of breakage events. This is important because by providing an efficient method of healing, the primary variable influencing the rate of $F O A^{R}$ is the rate of breakage. This aspect of the assay differs from 
another commonly used assay for end loss of chromosome $\checkmark$ [77], where healing can occur by a variety of mechanisms including telomere addition onto short naturally occurring GT sequences or recombination with other chromosomes, thus a change in rate could be due to an effect on either breakage or healing. Since relatively few genes affect the efficiency of telomere addition this ensures that in most cases an increase in YAC end loss is caused by increased breakage rather than increased healing. This has the added advantage that the effect of DNA repair genes on chromosome fragility can be measured. For example, we have shown that many genes required for double strand break repair pathways, such as homologous recombination and non-homologous end-joining, are important in facilitating repair at an expanded repeat and preventing chromosome end loss [117]. Nonetheless it should be noted that the YAC fragility assay only measures the events that result in end loss after resection of DNA sequence to the $\left(G_{4} T_{4}\right)_{13}$ telomere seed and telomere addition, not those that heal within the repeat without end loss, or those that fail to repair resulting in cell death. Marker loss assays underestimate the "true" rate of breakage but are useful for comparing between conditions.

In conclusion, the YAC fragility assay is a useful tool to quantitatively compare breakage rates of different sequences or of the same sequence in different conditions. As a powerful genetic assay, it can be used to identifying genes and pathways that are essential in preventing chromosome breakage. These studies have been instrumental in understanding how DNA repair and replication navigate difficult to repair and replicate sequences, which is important in our understanding of factors that influence chromosome fragility.

\section{Method to detect chromosomal rearrangements associ- ated with gene amplification}

It was only relatively recently that the large impact that structural genomic variation has on human genetic diversity and disease consequences has been fully appreciated $[118,119]$. In addition, the realization that the rate of spontaneous (de novo) copy number variation (CNV) is quite high in humans [120] strongly suggested that our genomes are far more structurally plastic than previously thought. This was a significant conceptual shift, considering that, until about 15 years ago the prevalent view was that the primary nucleotide sequence of the DNA molecule alone was the main source of genetic diversity. Since then, epigenetic inheritance and structural genomic variation have come into focus as fields of investigation that are critical to our understanding of how the traits of living organisms are determined and passed along through generations. Both fields offer great opportunities for discovery, with significant gaps in knowledge yet to be filled.

A reflection of the late emphasis on CNV mechanisms is that there are relative few experimental model systems that have been developed to study this problem [70, 121], particularly in the context of diploid genomes. One of the ways to interrogate fundamental aspects of CNV mechanisms is through the detection chromosomal rearrange- ments associated with gene amplification. The amplification assay described here is based on a copy number reporter cassette that contains both the SFA1 and CUP1 genes that confer gene dosage-dependent tolerance to formaldehyde (FA) and copper ( $\mathrm{Cu})$, respectively. These strains have full deletions of the native SFA1 gene, and a full deletion of the cluster of CUP1 genes. Therefore, the only copies of SFA1 and CUP1 in the genome are those present in the reporter itself, which can be integrated at different sites of interest in the $S$. cerevisiae genome. The current version of the system is the result of improvements to previously described approaches $[122,123]$. The earlier versions of this reporter contained the wild type SFA1 allele, which was useful for identifying clones containing extra copies of the reporter. This version was not very effective for the quantitative gene amplification detection since it allowed a high number of small confounding background colonies to grow on selective media [123]. The improved strains carry the gene amplification reporter

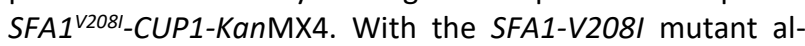
lele, the selection for additional copies of the reporter is much tighter, supporting the effective quantitative measurement of gene amplification rates. Nearly all of the colonies that grow on the selective media contain two or more copies of the reporter ( $<2 \%$ false positives). Most selected clones carry just two copies of the region where the reporter is inserted, rather than higher order amplifications commonly detected by earlier versions [122].

The diploid experimental strain shown in Figure 7A has one insertion of the reporter cassette on the right arm of chromosome 4 (Chr4). Parent cells carrying a single reporter copy are unable to grow on media containing high levels of FA and $\mathrm{Cu}$. However, mutant cells carrying a chromosomal rearrangement resulting in two or more copies of the reporter display resistance to the combined inhibitors, and therefore are able grow and form colonies on a Petri dish. These CNV-carrying clones are labeled FCR, for Formaldehyde and Copper Resistant (Figure 7B). In addition, the diploid parent strain contains an insertion of the TRP1 marker on the other homolog of $\mathrm{Chr} 4$ at a position allelic to the CNV reporter (Figure 7A). By selecting FCR clones on media containing FA and $\mathrm{Cu}$ but lacking tryptophan, it is possible to eliminate false positive cells that may duplicate the reporter through a mitotic crossover leading to LOH. This system is capable of identifying spontaneous chromosomal rearrangements resulting in a simple doubling of any region of interest in a diploid yeast cell's genome.

The use of this assay typically starts with a quantitative analysis of the gene amplification rate by counting the FCR colonies that grow on FA+Cu Trp drop-out plates. The basal (spontaneous) CNV rates range from $\sim 10^{-7}$ to $\sim 10^{-6}$ amplifications/cell division, depending on the genomic context of the reporter insertion. Changes to the basal CNV rates caused by environmental exposures or by genetic defects can be determined by exposing the parent cells to mutagens prior to selective plating or by mutating candidate genes of interest.

Once the amplification rates have been calculated, the next step in the analysis is the qualitative characterization 
of the chromosomal rearrangements leading to amplification of the reporter. This is done to obtain insight into the repair pathways involved in the formation of CNVs. The SFA1-CUP1 experimental system has two attractive features that distinguish it from other approaches used to study chromosomal rearrangements in budding yeast. First, the initiating DNA lesion that triggers the chromosomal rearrangement does not necessarily need to occur near the reporter cassette. In fact, the initiating lesion is often a double strand break in a different chromosome. Second, using diploid cells allows for sampling of a diverse spectrum of genome rearrangements, including large deletions that are not typically viable in haploids. These two key benefits are illustrated by the karyotype of clone FCR112. Pulse field gel electrophoresis (PFGE) showed a new chromosome of $\sim 1.3 \mathrm{Mb}$ in this clone (Figure $7 \mathrm{C}$ ), and arraybased comparative genomic hybridization (array-CGH) identified an amplification on the right arm of Chr4 associated with a deletion on the right arm of Chr7 (Figure 7D). The combined size of the preserved segment of Chr7 and the amplified segment of Chr4 is $1.3 \mathrm{Mb}$, suggesting that the new chromosome seen by PFGE is a Chr7/Chr4 nonreciprocal translocation (Figure 7E). Further tests (PCR, DNA sequencing) were then conducted to validate this

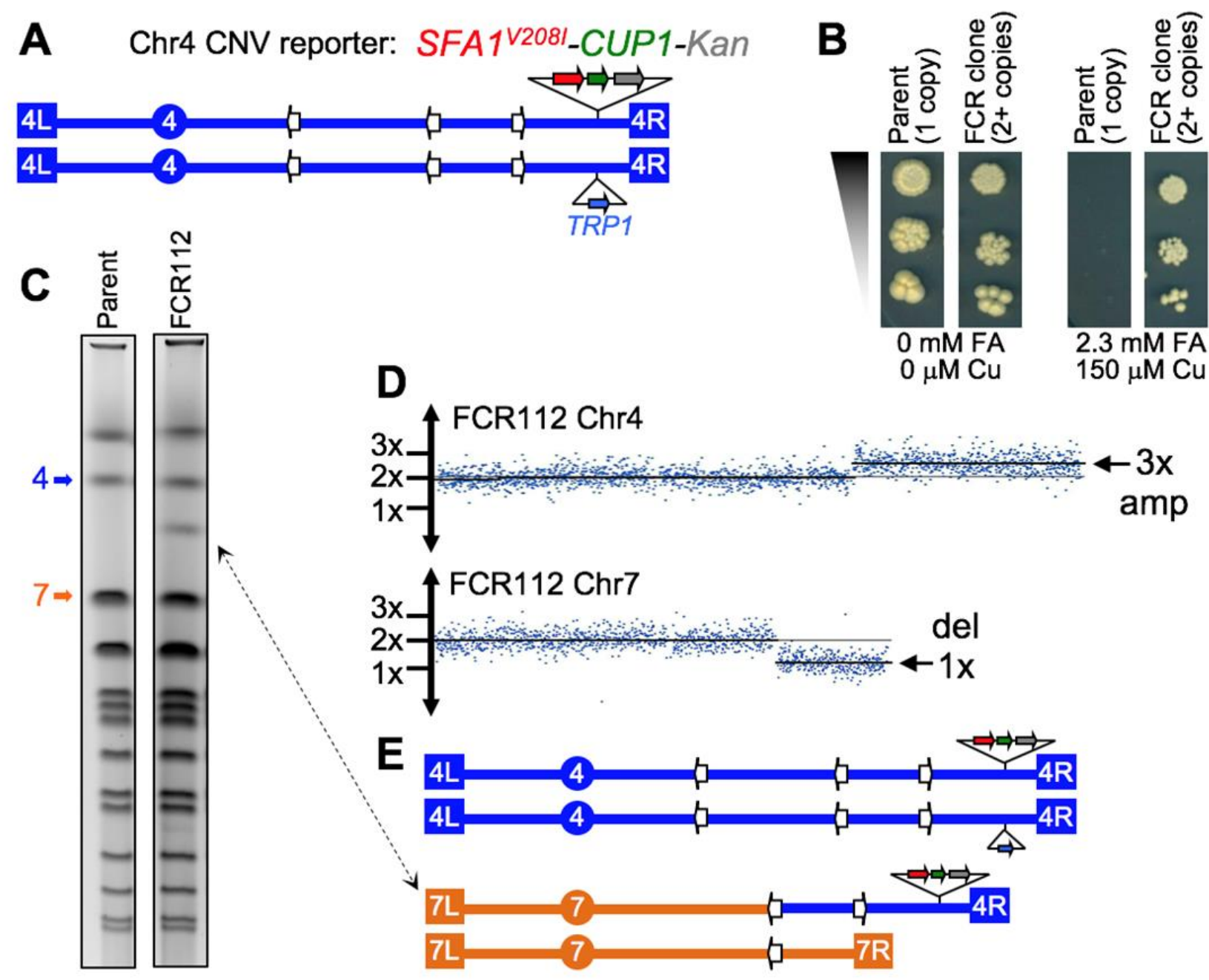

FIGURE 7: The diploid CNV assay. (A) Schematic map of Chr4 in a diploid test strain, showing telomeres (terminal boxes), centromere (circle), and Ty retrotransposon repeats (white arrows). One homolog has an insertion of the CNV reporter near the right end, the other has TRP1 at the allelic position. (B) Reporter dosage-dependent phenotype. Cultures were serially diluted and spotted on plates. The parent strain is unable to form colonies on selective media (Copper [Cu] and Formaldehyde [FA]), while an FCR clone displays full viability. (C) Pulse field karyotypes showing a $1.3 \mathrm{Mb}$ chromosomal rearrangement in CNV clone FCR112 (double-end arrow). The bands corresponding to normal Chr4 and Chr7 are indicated. (D) array-CGH data for FCR112. The plots show a $3 x$ copy number amplification region on the right arm of Chr4, and a $1 x$ deletion on the right arm of Chr7. The copy number breakpoints in both chromosomes correspond to the positions of Ty dispersed repeats. (E) Karyotype prediction for FCR112, showing a Chr7/Chr4 non-reciprocal translocation with a breakpoint junction at Ty sequences, suggesting a non-allelic homologous recombination mechanism of formation. Data credit to Ane Zeidler; full results to be described elsewhere (Zeidler, Argueso, et al., in preparation). 
prediction, and to determine the molecular CNV mechanism through fine breakpoint mapping. In this case, the mechanism was non-allelic homologous recombination (NAHR) between dispersed Ty1 retro-transposable repeats. The translocation detected in clone FCR112 was formed by a cellular pathway essentially identical to that recently reported to be a major driver of CNVs in humans - NAHR between human short dispersed repeats [124-126].

The SFA1-CUP1 system has been used to select and analyze more than 300 different FCR clones, derived both spontaneously, and induced by mutagens or derived from DNA repair defective mutant backgrounds. A broad range of $\mathrm{CNV}$ rates were observed, associated with a diverse spectrum of CNVs including segmental duplications, translocations, and aneuploidy. The molecular mechanisms inferred from DNA sequences present at breakpoints were NAHR in most cases, but also some created by non- or micro-homology pathways (NHEJ and Micro-homology Mediated Break-Induced Replication [MMBIR])[127]. The full results derived from this work will be described elsewhere (Zeidler, Stanton, Sharif, Argueso et al., in preparation).

Most of the cautionary notes that should be considered when using the SFA1-CUP1 reporter pertain to measures that must be taken to ensure that the selection is tight and that no false clones (without reporter amplification) are detected. The $\mathrm{FA}+\mathrm{Cu}$ selective plates should be incubated for five days at $30^{\circ} \mathrm{C}$ inside a large closed plastic box with a couple of moist paper towels inside. This is done to prevent the media from drying and cracking during the incubation. The first FCR colonies are visible after three days, the last ones appear on day 5. A high plating density can also lead to background growth. The number of cells per $\mathrm{FA}+\mathrm{Cu}$ plate should not exceed $10^{6}$ cells per normal size plate $(90$ $\mathrm{mm}$ diameter). If the frequency of amplification in the strain of interest is low, users need to plate multiple plates at $\sim 10^{6}$ cells/plate density. Plating at higher density $\left(>10^{7}\right.$ cells/plate) may in some cases lead to growth of false positive colonies that do not carry amplification of the reporter but manage to grow on selective media due to a "filtering" effect on top of a layer of dead cells. Finally, the formaldehyde in the plates tends to lose potency over time, therefore, fresh FA+Cu plates should be prepared the day before each plating experiment.

As described above, the combination of concentrations of FA and $\mathrm{Cu}$ is a critical parameter for the successful use of this assay. A given pair of concentrations may work very well for a specific strain (such as the example in Figure 7B) but would likely not be effective for a different one. This means the combination of concentrations needs to be reoptimized whenever using strains with the reporter inserted at a different genomic position. Likewise, if the reporter is transferred to a different genetic background it will be necessary to test a range of concentrations to find which are best suited to the new strain. Finally, specific users will presumably want to introduce mutations into their respective wild type strains (e.g. pol32 $\Delta$, rad51 $\Delta$, etc), and that will also require them to re-calibrate the ideal $\mathrm{FA}+\mathrm{Cu}$ concentration combinations. The re-optimization of the selection concentrations should be done to find the minimal concentration combination that fully inhibits the growth of a strain with one copy of the reporter. Once a few resistant clones are selected and confirmed to carry two copies of the reporter, they can then be used as positive controls to fine-tune the ideal concentrations for the assay. It is also sometimes necessary to adjust the FA concentrations when a new bottle of formaldehyde is received, as it may be slightly more or less potent than the previous stock solution.

In addition to pre-selection considerations, it is also important to keep in mind that it is common to observe different levels of $\mathrm{FA}+\mathrm{Cu}$ resistance between the clones that are selected in this assay. Clones with more than 2 copies of the reporter are always more resistant, but these multi-copy events are not very frequent (5-10\%). Most resistant clones only have two copies of the reporter. Most of the difference in resistance between clones is due to the nature of the chromosomal rearrangement present in each of them. Some rearrangements make the cells grow slowly (regardless of FA or $\mathrm{Cu}$ ) while others do not appear to interfere with growth.

In summary, the SFA1-CUP1 amplification assay offers attractive and distinctive features for the study of CNV mechanisms. However, it is somewhat laborious and more susceptible to leaky selection than other widely adopted approaches. Specifically, the haploid GCR assay is simpler and more robust from the experimental standpoint and is thus better suited for high throughput studies [128]. The SFA1-CUP1 assay is a highly valuable approach to take to answer a narrower set of questions, particularly those involving the role of ploidy. Used in combination, these two assays provide a powerful tool kit for answering a wider variety of questions about the mechanism of formation of chromosomal rearrangements.

\section{MOLECULAR BIOLOGY ASSAYS TO DETECT DNA BREAKS AND REPAIR}

Living cells constantly experience chromosomal lesions that derail the duplication of genome. Some of these lesions, such as DSBs, are particularly detrimental in that they, through chromosomal fragmentation, block both chromosomal replication and segregation and also cause loss of genetic information, - all resulting in cell death. Chromosomal DSBs threaten genome integrity and provoke rearrangements that are the hallmark of cancer cells.

Breaks can be repaired by two fundamentally different pathways: NHEJ or HR. NHEJ is intrinsically mutagenic, since most events that ligate ends back together result in deletions of varying size or in small insertions. NHEJ itself can be subdivided into two pathways, termed "classic" and "alternative" (or microhomology-mediated end-joining) that differ both in their genetic requirements and especially in the small number of bases that need to be paired at the junctions.

In contrast, HR relies on repairing the DSB by copying sequences from an intact template. The most precise of the HR processes is gene conversion by synthesisdependent strand annealing (SDSA), in which the break is 


\section{BOX 2: MOLECULAR BIOLOGY ASSAYS TO DETECT DNA BREAKS AND REPAIR}

Detection and quantification of DNA breaks by pulsed field gel electrophoresis | Assays for physical detection of DNA breaks. The methods can detect low abundance of breaks and be quantitative.

Introduction of site-specific DNA breaks and analysis of repair | A combination of genetic and physical assays can monitor break formation and repair in real time.

Quantification of strand-specific single-stranded DNA | Assays for measurement of single stranded DNA formation in real time. These assays measure DNA unwinding or resection at a double-strand break.

Detection of toxic recombination intermediates | Recombination intermediates arising from excess single-stranded DNA can become toxic if not correctly resolved. These assays monitor formation of these toxic intermediates.

Detection of hypermutable single-strand DNA in living cells | Single-stranded DNA that occurs during the repair process of a DNA lesion can be a source of mutation, arising from error-prone replication across a damaged template. The sources of single-stranded DNA and the mutagenic processes can be detected by genetic reporters and physical methods.

repaired by a short "patch" of DNA copied from a template (gene conversion), that can be associated or not associated with crossover. A distinct, but related process, involving the formation of a double Holliday junction intermediate $(\mathrm{dHJ})$, also produces a patch of newly copied DNA but often is accompanied by reciprocal exchange between the DNA segments containing the donor and recipient. SDSA and $\mathrm{dHJ}$ mechanisms require that both ends of the DSB share sufficient homology with the donor to effectively repair. If only one end shares enough homology, a BIR process is launched, copying a template chromosome all the way to its telomere. BIR thus can result in non-reciprocal translocations. A fourth HR process is single-strand annealing (SSA) in which a deletion is formed by $5^{\prime}$ to $3^{\prime}$ resection of DSB ends and annealing of homologous sequences flanking the DSB. Much of our understanding of DSB formation as well as of both NHEJ and HR has come from studies in bacteria and yeast.

In this section, the molecular biology assays in $E$. coli and yeast designed to detect DSBs and their repair are described (Box 2).

\section{Detection and quantification of DSBs by pulsed field gel electrophoresis}

When DSBs are initiated by a site-specific endonuclease, it is relatively easy to detect their formation by using Southern blot analysis and a radioactively labeled probe specific for the DSB site (reviewed in [129-131]). DSBs initiated at random places in genomic DNA are more difficult to detect. They are frequently reported by methods like neutral sucrose gradient centrifugation [132], neutral elution [133], comet assay [134], or DNA diffusion assay [135]. Unfortunately, these methods are either suboptimal for the quantification of the real density of DSBs or are purely qualitative. In contrast, PFGE offers a straightforward way to quantify DSBs with at least five orders of magnitude dynamic range (if DNA is radiolabeled and phosphorimaging detection is used) [136, 137]. The range of resolution and linearity of quantification makes the technique especially useful for DNA damage studies of prokaryotes harboring circular genomes. Here the detection of DSBs by PFGE is described.

PFGE was developed to resolve chromosome-sized linear DNA molecules and has been used extensively since its inception in genome characterization [138-143], and epidemiological studies [144-146]. The technique is highly versatile and can be used to separate linear DNA ranging from $30 \mathrm{kbp}$ to $12 \mathrm{Mbp}[147,148]$, making it an excellent tool for general genome characterization in bacteria and low eukaryotes, and the standard approach for molecular genotyping, when used in combination with rare-cutting restrictases [149, 150]. Furthermore, the technique is uniquely suited for visualization and quantification of subchromosomal fragments in organisms harboring circular chromosomes, like most prokaryotes, because not only circular DNA in general moves slower in agarose than linear DNA of the same size [151-154], but also circles longer than $30 \mathrm{kbp}$ do not even enter pulsed-field gels [155, 156], keeping the intact chromosomal DNA in the wells [157]. Previously, PFGE has been used to visualize randomly distributed DSBs induced by gamma-irradiation in the yeast $S$. cerevisiae [158]. Here we describe the use of PFGE to characterize formation of DSBs in bacteria. In particular, PFGE is used to characterize spontaneous chromosomal fragmentation due to various defects in the chromosomal metabolism [159-165] or fragmentation induced upon exposure of bacterial cells to various clastogens [166-170].

Continuous electric field resolves DNA molecules based on size as long as they are small and are able to sieve through agarose gel matrix. As the size of DNA increases, it begins to reptate (moving in a snake-like fashion), causing decrease in sieving and making DNA move with a sizeindependent velocity. The size-velocity correlation is ultimately lost in regular agarose gels when the size of DNA reaches $50 \mathrm{~kb}$ [133]. Extremely low concentration of agarose separates DNA up to $170 \mathrm{kbp}$ and even $\sim 450 \mathrm{kbp}$ in length, but the fragility of gels and long-time of electrophoresis makes these gels impractical $[171,172]$; besides, they still cannot separate chromosome-sized molecules. PFGE overcomes the limitation of continuous electric field gel electrophoresis by periodically changing the directions 
of the electric field. Such a maneuver forces DNA molecules to change orientations, as they move through the agarose, resulting in efficient, size-dependent separations of linear DNA pieces as long as $12 \mathrm{Mbp}$ [147]. The movement of DNA through the PFGs depends upon a variety of factors including field strength, pulse time, gel concentration, and time of electrophoresis. By varying these conditions, PFGE can be customized to resolve specific size ranges of DNA molecules. As circular DNA, including large plasmids, stays in wells, while the linear fragments enter the gel and resolve based on their sizes, the separation of linear and circular DNA during PFGE becomes a binary system (Figure $\mathbf{8 A}$ ). The separation is especially effective for the smaller molecules (20-500 kbp), the sizes of which can also be used to calculate the density of DSBs in the chromosomes [170].

The measurement of chromosomal fragmentation is performed in three steps. In the first step, chromosomal DNA of the growing cultures of $E$. coli is labeled with ${ }^{32} \mathrm{P}$-orthophosphoric acid, and bacterial cells are embedded in agarose plugs and lysed to release the cell constituents. In the second step, the plugs are loaded onto agarose gels and subjected to PFGE using the conditions specific for separation of linear DNA in a particular (broad) size range (for example, 20-600 kbp, or 300-3,000 kbp, or 1-10 Mbp).
In the final step, the gels are dried under vacuum, subjected to phosphor-imaging and quantified using phosphorimaging software.

Using PFGE, spontaneous chromosomal fragmentation was detected in $\operatorname{rec} B C D$ mutant, in which DSBs persist [161, 166]. Combining $\triangle s e q A$, polA12(Ts), and ligA251(Ts) mutations with $\operatorname{rec} B C D$ defects increased the chromosomal fragmentation (Figure 8B), revealing the cellular roles of SeqA, DNA polymerase I and DNA ligase in the avoidance of spontaneous DSBs in E. coli [167].

Treatment of growing cultures of $E$. coli with clastogens also causes chromosomal fragmentation that can be detected and quantified by PFGE (Figure 8B, C). As expected, increasing the amount of damage, either by increasing the dose or time of exposure, led to higher number of DSBs, resulting in ever smaller sub-chromosomal fragments (Figure $\mathbf{8 C}$ ). Interestingly, clastogens that affect only one strand of DNA, such as UV, also cause fragmentation of chromosomes in growing cells [166] (Figure 8B).

PFGE is an excellent tool to quantify chromosomal fragmentation resulting from DNA damage. However, the technique has its own (minor) limitations. First, branched DNA structures, including replication and repair intermediates, do not enter the gel [173]. If a significant fraction of these is suspected, treatments of DNA in agarose plugs
A

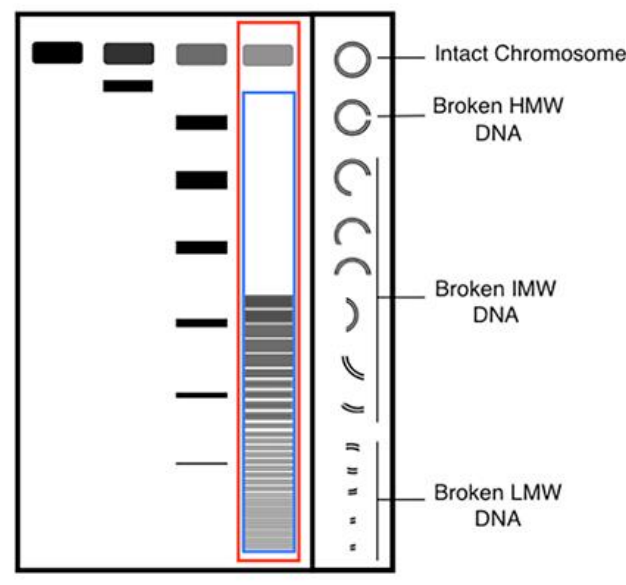

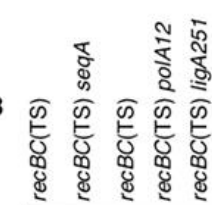

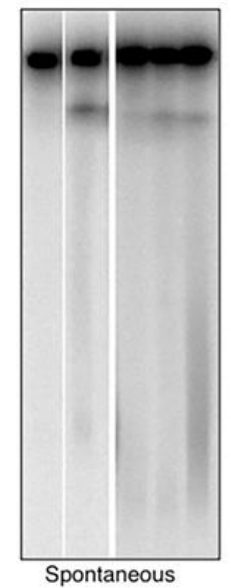

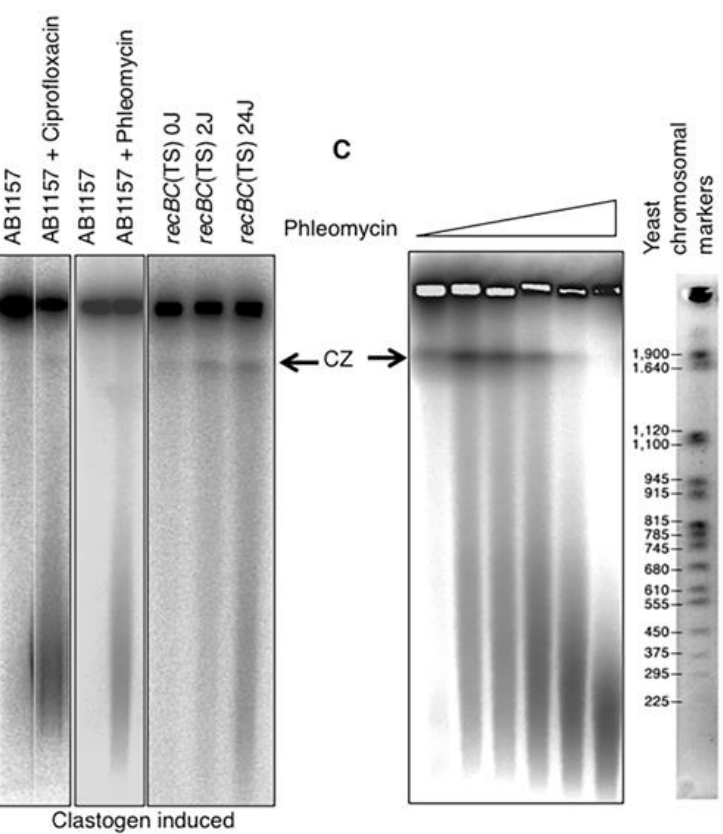

FIGURE 8: Fragmentation of bacterial chromosomes under various conditions. (A) Schematic representation of size-dependent migration of linear DNA through PFGs. Note that the intact circular chromosome stays in the wells. Chromosomal fragmentation is quantified as the percentage of the total signal (well+lane, red rectangle) that enters the lane (blue rectangle), that is: [Signal in lanes $x 100 /$ total signal (well+lane)]. HMW, high molecular weight ( $\geq 2 \mathrm{mbp}$ ); IMW, Intermediate molecular weight (0.2 - $2.0 \mathrm{mbp})$; LMW, low molecular weight ( $\leq 0.2 \mathrm{mbp}$ ). (B) Spontaneous and clastogen-induced chromosomal fragmentation in E. coli. Typically, chromosomal fragmentation is detected in the recBC mutant, deficient both in repair of DSBs and in degradation of linear DNA. However, when fragmentation is massive, it can be detected in wild type cells (such as ciprofloxacin- or phleomycin-induced fragmentation in $A B 1157$ in these examples). Spontaneous fragmentation is measured under conditions when the $\operatorname{rec} B C$ (Ts) mutants carry additional defects in SeqA, DNA polymerase I or DNA ligase [165, 167]. Clastogen-induced fragmentation is observed upon exposure to phleomycin and ciprofloxacin. Ultraviolet light,

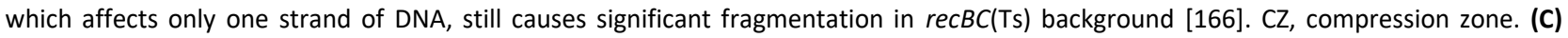
Pulsed field gel electrophoresis can be used to calculate the density of breaks in the chromosomal DNA if the average size of the resulting chromosomal fragments is measured. 
with enzymes, such as T7 debranching endonuclease, may facilitate their migration into the gel. Second, presence of nicks and gaps may cause entrapment of DNA in the wells and may require longer electrophoresis at reduced field strength to facilitate their migration in the gels [167]. Third high molecular weight linear DNA with nicks has been shown to break during PFGE, thereby inflating the density of DSBs [167]. In some cases, when sharp banding pattern is lost due to degradation, inclusion of thiourea in electrophoretic buffer may help to preserve the integrity of DNA [174]. An important point when lysing bacteria during the preparation of the chromosomal plugs is to minimize artefactual fragmentation during lysis. We have recently shown that inclusion of a non-specific RNase treatment during preparation of plugs causes extremely high spontaneous fragmentation by activating the surface endonuclease of E. coli, Endo I [175]. Therefore, RNase treatment should not be used during preparation of plugs, at least with bacterial cells.

Currently PFGE, in combination with labeling chromosomal DNA with ${ }^{32} \mathrm{P}$, is a gold standard for determination of chromosomal fragmentation in $E$. coli, offering excellent range of separation and exceptional dynamic range of quantification. Minor changes in plug preparation make the technique equally suited for use with Gram-positive bacteria, including pathogenic Mycobacteria. Since chromosomes can be also radiolabeled with ${ }^{3} \mathrm{H}$ thymidine, the technique can be used without phosphorimaging, by simply slicing the gels and subjecting gel slices to scintillation counting [157].

\section{Analyzing the repair of site-specific chromosome breaks in yeast}

Induction of site-specific DSBs and analysis of their repair in budding yeast

Much of our understanding of both NHEJ and HR has come from studies in the budding yeast, $S$. cerevisiae, where DSBs can be induced in virtually all the cells of the population in a highly synchronous fashion, by placing the sitespecific $\mathrm{HO}$ endonuclease under the control of a galactoseinducible promoter. In most such experiments, GAL::HO is integrated at the ade3 locus [176]; $\mathrm{HO}$ induction is rapid once $2 \%$ galactose is added to cells grown in medium containing raffinose or lactate as a carbon source. The most widely used assay is the switching of budding yeast matingtype genes, from MATa to MAT $\alpha$ (shown in Figure 9A) or from MAT $\alpha$ to MATa, using the opposite donor sequence, HMRa (not shown) [177]. The efficiency of HO-induced cleavage can be monitored on a Southern blot by using a MAT-distal probe (Figure 9B).

In cells in which both the HML $\alpha$ and HMRa donor loci are deleted, cells will die unless repair occurs through NHEJ. Because $\mathrm{HO}$ endonuclease is rapidly degraded, by briefly inducing $\mathrm{HO}$, one can examine the perfect re-ligation of the 4-nt, 3' overhanging ends by the re-formation of the Styl MATa band, as well as by measuring viability. When $\mathrm{HO}$ expression is continuous, then viability drops to about
$0.2 \%$ and all the survivors have an insertion or deletion, created by NHEJ, altering the cleavage site [178].

In strains that carry the $H M L \alpha$ and HMRa donors, it is easy to follow repair by homologous recombination real time, by a combination of genetic assays and physical monitoring of DNA (Southern blots and PCR). As shown in Figure 9B, HO cleavage produces a smaller Styl fragment homologous to the MAT-distal probe. After a delay of an hour, the gene conversion product, MAT $\alpha$, becomes visible, because the $\alpha$ sequences lack a Styl site present in the aspecific sequences and hence the product is a larger, easily identified band; other restriction sites can also be exploited [179]. By similar strategies, and inserting HO cleavage sites in other genomic locations, one could follow the kinetics of SSA [180] or BIR [181]. A galactose-inducible I-Scel endonuclease, assayed in the same ways, demonstrated that the outcomes were not dependent on a particular nuclease [182].

The consequences of deleting different repair and replication-associated genes can then be assessed. It is also possible to identify intermediates of repair, such as the $5^{\prime}$ to $3^{\prime}$ resection of DSB ends [183]. The advent of chromatin immunoprecipitation made it possible to follow the recruitment of the Rad51 recombination protein and other proteins to the site of the DSB [184]. These results inaugurated the age of "in vivo biochemistry" ( reviewed in [185]).

Over the past 3 decades, various studies have defined the roles of many DSB repair and replication proteins in each of the processes enumerated above. These studies have been recently augmented by using inducible Cas 9 site-specific nucleases to cleave DNA. As genetic modifications have become easier, several other questions have been explored in greater detail.

Using a BIR system in which homology is confined to 108 bp it has been asked: How tolerant is the Rad51 filament to carry out strand exchange with divergent substrates? It was found that even substrates with every $6^{\text {th }}$ base mismatched are able to be used, albeit at only about $5 \%$ of the rate of fully matched sequences [186].

By placing donor sequences, sharing $1 \mathrm{~kb}$ homology on either side of a DSB, it was enquired how the efficiency of DSB repair depends on the proximity, as measured by contact probability using chromosome conformation capture data. It was shown that there is a very strong correlation between the likelihood of collision and the efficiency of repair. Other performed experiments suggested that only about one in four collisions is likely to lead to a successful repair event $[187,188]$.

By changing the lengths of homology shared by the HOcleaved target and a donor and by exploiting a "Recombination Enhancer" (RE in Figure 9A) that binds near the DSB and thus drags a nearby donor near the break, it was shown that the ability of a 35-bp homologous sequence to initiate repair was greatly improved by tethering a distant intrachromosomal donor near the DSB [189].

By deleting or overexpressing Sgs $1^{\mathrm{BLM}}$ and Mph1 $1^{\mathrm{FANCM}}$ helicases it became possible to demonstrate their synergis- 
tic and distinct roles in promoting or discouraging BIR and "second end capture" during gene conversion [189, 190].

By creating a counter-selectable reporter gene in a donor locus it was discovered that gene conversion repair of a DSB is 1000 time more mutagenic than simple replication of the same sequences [191, 192]. It was demonstrated

A
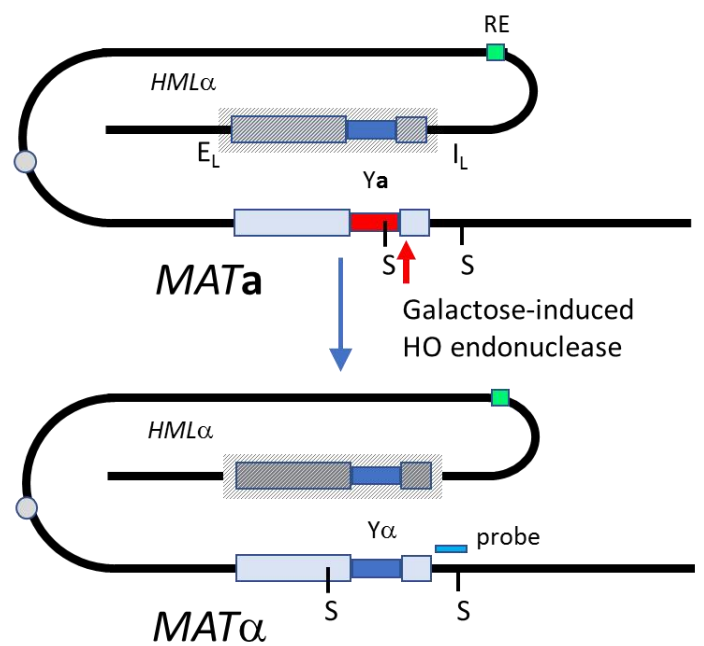

B

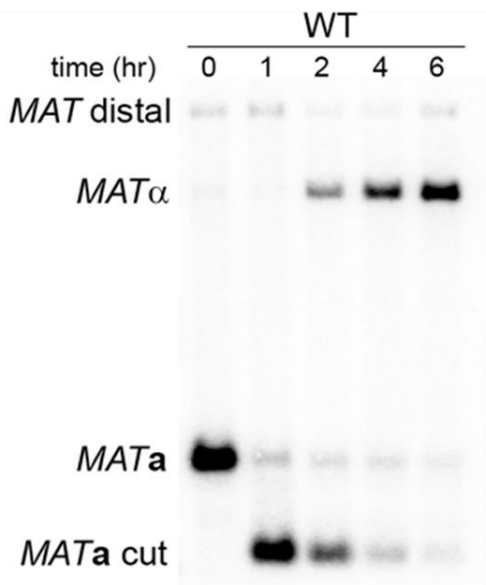

FIGURE 9: Mating-type gene switching in budding yeast. (A) Switching of MATa to MAT $\alpha$ by homologous recombination between the $\mathrm{HO}$ endonuclease-cleaved MATa locus and the $H M L \alpha$ donor locus. Part of chromosome III is shown. HML $\alpha$ and MATa are each about $100 \mathrm{~kb}$ from the centromere (circle). HML $\alpha$ and the other donor, HMRa (not shown, at the opposite end of the same chromosome), are bounded by silencer sites ( $E$ and I) that create a highly ordered nucleosome structure (hatched lines) that makes the donors transcriptionally silent and prevents $\mathrm{HO}$ cleavage at the same $\alpha$ or a sequences that are present at MAT. A Recombination Enhancer (RE) facilitates the use of $H M L \alpha$ through Fkh1 protein binding both to RE and to sites near the DSB. Styl sites are indicated by S. (B) Kinetics of MAT switching. The cleavage of MATa is complete after inducing $\mathrm{HO}$ endonuclease for $60 \mathrm{~min}$. Cleavage and subsequent steps are assayed on a Southern blot, using a MATdistal sequence as probe (green). HO-induced cleavage of MATa is followed by the appearance of MAT $\alpha$, which has different Styl restriction sites and is thus easily distinguished from MATa. Here, the $H M L \alpha$ donor also harbors a single-base pair mutation that prevents subsequent $\mathrm{HO}$ cleavage. that the principal causes of these mutations were replication-slippage and dissociation/reassociation of DNA polymerase $\delta$ during the copying of the donor sequences. Similar template jumps are found in BIR [193].

With the advent of CRISPR/Cas9 it is possible to compare and contrast the consequences of generating (usually) blunt ends with $\mathrm{HO}$ cleavages with 4-bp 3' overhanging ends. Such studies are now ongoing.

DNA DSB induction and repair in fission yeast

Synchronous induction of a single genomic DSB offers one of the most effective ways to follow DNA recombination in cells. In budding yeast, induction of $\mathrm{HO}$ and I-Scel endonucleases was used for decades to follow DSB repair by NHEJ and HR [194]. These studies uncovered molecular mechanisms of DSB repair pathways and the function of proteins involved. Similar approaches were subsequently developed in other organisms including human however often with less efficient and less synchronous DSB induction.

Here we describe DSB-induced recombination assays in fission yeast, a model organism that offers some advantages when compared to budding yeast. Initial DSB inducible recombination assays with $\mathrm{HO}$ endonuclease induction under a thiamine-regulated $n m t$ promoter, uracil-regulated urg1 promoter [195], or glucose-repressed inv1 promoter were inefficient in terms of synchrony of DSB induction when compared to budding yeast assays. An additional complication of these assays is that induction of the DSB requires a switch of growth media and thus alteration of cellular metabolism that could impact DSB repair analysis. Recently, the Sanders lab developed an assay where the homing endonuclease I-Ppol from the slime mold Physarum polycephalum is placed under the control of an anhydrotetracycline (ahTET)-inducible promoter [196]. Addition of anhydrotetracycline to the growth medium inactivates the Tet repressor leading to high promoter activity (Figure 10A).

The drawback of I-Ppol enzyme usage is that its recognition sequence lies within rDNA repeats (Figure 10B). While cleavage within rDNA can be used to follow DSB repair within repetitive sequences [197], in most studies it is undesirable feature. To acquire cells resistant to I-Ppol enzyme cleavage within rDNA, cells are plated on anhydrotetracycline containing plates. A small number of cells $(\sim 1 / 1000)$ survive rDNA cleavage via a typical single nucleotide change within the I-Ppol cleavage site [198]. Sequencing of this region is then essential to confirm that the rDNA carries the mutation within the I-Ppol cleavage sites (Figure 10B). To avoid rDNA cleavage, one can also use I-Scel enzyme instead of I-Ppol. Such an assay was recently developed by the Runge lab with codon-optimized I-Scel endonuclease carrying two nuclear localization signals (NLS) at the N-terminus. In this new system I-Scel is also under the control of an anhydrotetracycline (ahTET)-inducible promoter and cleavage by I-Scel is nearly as efficient as with IPpol enzyme [199].

An example of the synchronous and efficient induction of a DSB by I-Ppol and analysis of DSB ends resection are shown in Figures 10 C-D. In this assay, the TetO7::I-Ppol 
cassette is inserted at the leu1 locus and a single DSB is generated at the lys1 locus as described [196]. DSB induction and resection are followed using Southern blots and probes specific for the cleavage site or $3.2 \mathrm{~kb}$ away from the DSB ends. We note that induction of DSBs is faster in EMMG media when compared to rich media and best at low cell density $\left(2-4 \times 10^{6} / \mathrm{ml}\right)$. Cells are collected before DSB induction and every 30 minutes after induction. DNA is isolated by standard phenol-chloroform extraction, digested with EcoRI and separated on $0.8 \%$ agarose gel, then transferred to a positively charged membrane and subjected to Southern blot analysis with probes corresponding to sequences at the DSBs. The kinetics of resection directly correlates with the kinetics of disappearance of Southern blot bands corresponding to the probes used.

We also note that the strain described above cannot be used efficiently to study NHEJ associated with errors. Cells plated on tetracycline containing plates induce I-Ppol enzyme permanently and normally only cells that repair the DSB by an NHEJ event that alters the I-Ppol cleavage site should be able to survive. Here, however, Tet07::I-Ppol was inserted at the leu1 locus with a pDUAL plasmid that generates two direct leu1 gene fragments [200]. Recombination between two leu1 fragments leads to a loss of the IPpol cassette and therefore growth on tetracycline plates. The frequency of this spontaneous event is within the range of error associated with NHEJ and therefore the system is not best suited to study error-prone NHEJ (Ira's lab unpublished results).

Another drawback of this system is that a fraction of cells induce I-Ppol and DSBs without the addition of tetracycline, which makes establishing a repairable system difficult. A similar problem was observed with repairable recombination assays using $\mathrm{HO}$ under the control of the urg1promoter (e.g. [201]). To avoid such issues, a repairable system where the repair of a DSB by SSA leads to the elimination of an essential gene, gtr2 and consequently cell death was designed (Figure 10C). Thus, cells in which a DSB is induced prior to tetracycline addition are eliminated from the population. The system presented in Figure 10D is ideal to compare the efficiency and kinetics of DSB repair by SSA in fission yeast. A single DSB is induced at an I-Ppol cleavage site inserted within a partial sequence of phage lambda integrated at the $\arg 1$ locus on chromosome III. It is repaired by SSA using an identical lambda sequence (755 bp) located $21 \mathrm{~kb}$ upstream. To eliminate Rad51mediated strand invasion, the analysis is performed in rad51 cells.

An alternative way to limit background expression of IPpol could be accomplished by incorporation of a DSR sequence (Determinant of Selective Removal) at the 3' UTR of the I-Ppol gene that directs background transcripts through the MTREC complex to the nuclear exosome for degradation [197]. This recently described system of I-Ppol induction is similar to the one presented in Figure 10A, with the endonuclease under the control of the anhydrotetracycline (ahTET)-inducible promoter. However, this alternative scenario uses a modified $\mathrm{CYC} 1$ promoter coupled with seven Tet operator sequences (tetO7) repressed by constitutively low expression of TetR (TetR-tup11). The same TetO7-CYC1 promoter also controls expression of ncRNA-8xDSR. Addition of ahTET to the media releases TetR from the promoters of I-Ppol-4xDSR and 8xDSR and high amounts of ncRNA-8xDSR compete for MTREC pathway components, thus stabilizing the I-Ppol-4xDSR nuclease. Rapid DSB formation within rDNA or within a separately inserted I-Ppol recognition site is observed in this system [197].

As presented here, a number of new DSB-inducible recombination assays were developed recently in fission yeast. These assays will facilitate studies of DSB-induced recombination in fission yeast.

Quantification of strand-specific single-stranded DNA in $D N A$ repair by real time $P C R$

Single-stranded DNA (sSDNA) is an obligatory intermediate in DNA replication and transcription events and naturally forms at telomeres through unwinding of duplex DNA by DNA helicases or by nucleolytic degradation of one strand [202]. ssDNA is also generated in all types of DNA repair processes including nucleotide excision repair (NER), base excision repair (BER) and DSB repair (DSBR). Moreover, SSDNA gaps arise as a part of DNA damage tolerance when DNA lesions impede progression of replication forks. To detect and measure ssDNA intermediates and their strandspecificity within cells would be essential to monitor progression of all these events and to understand the underlying molecular mechanisms. Traditional methods to detect intracellular ssDNA typically involve Southern hybridization or immunoblotting which are time-consuming and require a substantial amount of materials and reagents. Here we describe a convenient, quantitative and highly sensitive way to detect strand-specific ssDNA in the genomic DNA in $S$. cerevisiae cells. This method could be readily adapted to other organisms.

In principle, restriction endonucleases can only digest duplex DNA molecules while leaving ssDNA intact. By exploiting this differential enzyme digestion pattern, this strategy quantifies the percentage of SSDNA at a specific locus in the genomic DNA by real-time PCR. To discriminate strand-specificity of the ssDNA generated, i.e. the Watson or the Crick strand, a 24-mer oligonucleotide complementary to the restriction enzyme recognition site on either the Watson or Crick strand is included in the reaction. Then based on the enzyme digestion and PCR patterns we can deduce the strand of origin. For instance, if the SsDNA corresponds to the Watson strand, inclusion of complementary oligonucleotides will render it cleaved by restriction enzyme and SsDNA from the Watson strand will no longer be amplified by PCR using primers flanking the restriction site (Figure 11A). To avoid excess levels of annealing oligonucleotides being used as primers to generate PCR products in the real-time PCR reaction, four mismatched nucleotides are placed at the $3^{\prime}$ end of the oligos, effectively blocking their contribution as PCR primers.

Several labs have used this or similar strategies [203205] to measure the formation of SSDNA at a site-specific HO-induced DSB, a process called DNA end resection, dur- 
A

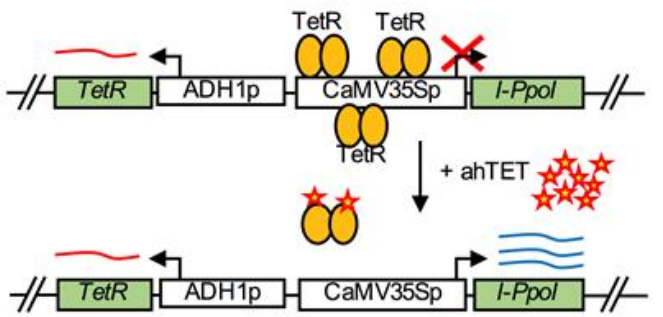

B

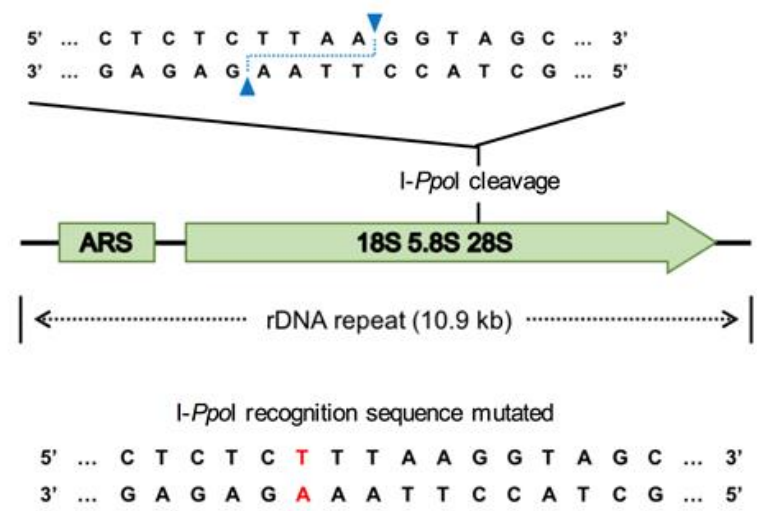

C Wild-type cells
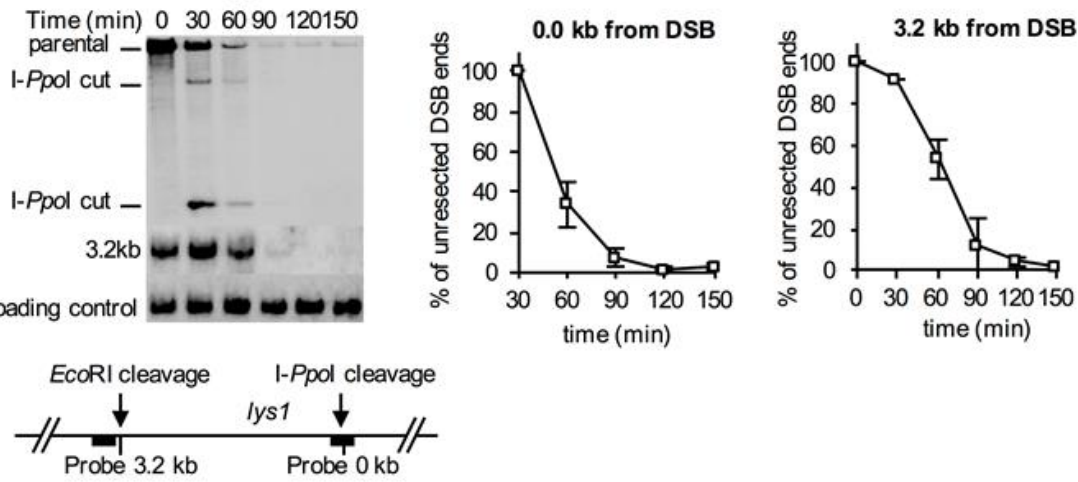

Chromosome I

D

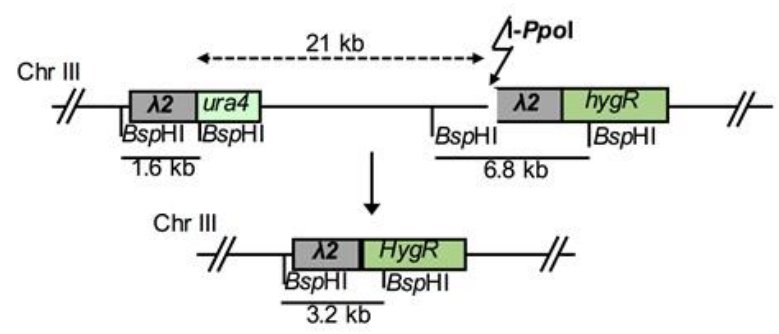

FIGURE 10: DSB induction and repair in fission yeast. (A) Inducible I-Ppol system. I-Ppol is under the control of an anhydrotetracycline (ahTET)-inducible promoter. The ADH1 promoter drives constitutive expression of a tetracycline repressor, TetR. TetR binds to and represses the promoter of the plant viral cauliflower mosaic virus $35 \mathrm{~S}$ (CaMV35S) carrying three Tet operators (binding site for TetR). Addition of tetracycline inducer (yellow stars) inactivates TetR and thus activates I-Ppol expression. (B) Diagram showing the I-Ppol recognition sequence, cleavage site within rDNA and typical sequence change in cells resistant to I-Ppol cleavage (ARS, autonomously replicating sequence). (C) Analysis of DSB induction by I-Ppol and resection by Southern blot, and quantification of kinetics of resection at DSB ends and at $3.2 \mathrm{~kb}$ from the DSB. Diagram shows I-Ppol cleavage site at the lys1 locus. (D) Diagram of the SSA assay between two partial lambda sequences. Southern blot analysis of SSA. Lambda sequence was used as a probe.

ing which both ends from a DSB undergo $5^{\prime}$ to $3^{\prime}$ degradation by a group of nucleases, generating ssDNA with $3^{\prime}-\mathrm{OH}$ overhangs [206]. DNA end resection is a key step in the initiation and regulation of DNA recombination and the DNA damage response in all eukaryotic cells [202]. Compared to Southern blot hybridization, the PCR based assay is fast and requires fewer cells to reliably detect ssDNA at given locations. Furthermore, from a single digestion reaction, it is possible to monitor the ssDNA formation at multiple locations in the genome and deduce the rate of resection based on the progressive formation of SSDNA at several distal locations. One routinely used restriction enzyme is the BsaJ 1 which recognizes $C^{\wedge} C N N G$ G sequence as a frequent cutter. Previously, the 
Diffley lab used BstU1(CG_^CG) for the equivalent PCR based assay [204]. Since BsaJ1 cuts at $60^{\circ} \mathrm{C}$, the annealing and enzyme digestion can be performed as a single step to assess the strand specificity of ssDNA.

The sensitivity of this PCR based ssDNA detection assay depends on the efficacy of the enzyme digestion because residual DNA that remains undigested in the reaction can increase background signals and thus limit the sensitivity of the assay. It was found that BsaJ1 is very efficient in restriction digestion and well suited for the assay. Using the genomic DNA samples isolated from G1 arrested wild-type cells, only $0.1-0.4 \%$ background levels are detected of PCR signal at any given location (Figure 11B). Notably, the sum of ssDNA from Watson and Crick strands as determined by annealing of corresponding oligonucleotides is consistently close to that without adding annealing oligonucleotides, with the ratio of ssDNA from Watson and Crick strands corresponding to almost one. The result suggests that the 0.1-0.4\% background levels of PCR are not derived from undigested DNA but from actual ssDNA which is likely generated spontaneously either in vivo or during genomic DNA isolation. The result also suggests that the complementary annealing of oligonucleotides and subsequent enzyme digestion are very efficient.

In addition to analyzing resection at DNA breaks, this technique was also used to determine the levels and the strand specificity of ssDNA in cells suffering from replication stress to study the roles of DNA damage tolerance (DDT) mechanisms in post-replicative gap repair in budding yeast cells. Multiple types of lesions on DNA templates can stall the ongoing DNA synthesis activity of high fidelity DNA polymerases, and the lesions must be tolerated/bypassed to ensure that replication is completed in time [207]. Evidence emerges that DNA synthesis can restart downstream of a lesion on the leading strand template by re-priming and a ssDNA gap is left behind. Similarly, on the lagging strand template, a ssDNA gap could be formed due to an interruption of Okazaki fragment synthesis when the lagging strand polymerase encounters a blocking lesion [207]. The resulting ssDNA gaps are then filled in by Rad18dependent DDT mechanisms in a post-replicative manner. The DDT pathways can be further divided into two branches, error-prone translesion synthesis (TLS), and Rad5, Ubc13-dependent template switching [208, 209]. Importantly, ssDNA is the common substrate of both DDT pathways. To understand DDT mechanisms, ssDNA formation in $S$ phase from cells exposed to UV lesions was examined using a PCR based assay and this allowed to analyze the role of Rev3 or Ubc13 in the gap repair. Briefly, NER-deficient rad14 $\Delta$ cells were arrested in G1 phase, treated with $10 \mathrm{~J} / \mathrm{m}^{2} \mathrm{UV}$ and released cells into $\mathrm{S}$ phase. Then the formation of SsDNA was measured at multiple locations near the early firing ARS305 replication origin (Figure 11C). By determining strand specificity of SSDNA, it was also examined whether ssDNA accumulates from the leading or lagging strand (Figure 11C). As expected, the sum of SsDNA at leading and lagging strands is nearly identical to the total ssDNA detected, again reinforcing that the complementary annealing of excess oligonucleotides and enzyme digestion are very efficient in this assay. The results suggest that Ubc13 suppresses the formation of ssDNA in both leading and lagging strands in S phase (Figure 11C).

Despite many advantages of the PCR based ssDNA detection assay described herein, the method could underestimate the amount of intracellular SSDNA at a given locus if ssDNA formation relies on the temporary unwinding of duplex DNA such as that formed during undisturbed DNA replication or transcription. Unwound DNA will simply reanneal during genomic DNA isolation and is still cleaved by restriction endonuclease. It was estimated that the lower limit of detection in this PCR assay is $\sim 0.1 \%$ due to the spontaneous formation of ssDNA in the genomic DNA isolated from G1-arrested cells. Levels of SsDNA below this threshold will not be detected by the current technique. The use of 20-24-mer oligonucleotides also limits determination of the strand specificity of ssDNA less than 20 nucleotides long.

In summary, we have presented here a fast, sensitive and cost-effective assay to measure the amount of ssDNA at defined locations in budding yeast cells. This technique can also determine the strand specificity (either Watson or Crick strand) of the ssDNA. Since ssDNA formation is a common feature in many DNA transactions, this assay should have wider applications and could also be applicable to analyzing the equivalent processes in other organisms including human cells.

Detection of toxic recombination intermediates in the yeast S. cerevisiae

SSDNA is a recombination intermediate that promotes homology search and strand invasion. However, unregulated accumulation of excessive SsDNA can also lead to the formation of structures containing both the broken and intact DNA molecules, called toxic intermediates, which if not resolved are detrimental for the cell. Accumulation of toxic recombination intermediates leading to cell lethality have been observed in various yeast mutant backgrounds, including $\operatorname{sgs} 1 \Delta$, top $3 \Delta$, and $\operatorname{srs} 2 \Delta[39,210-213]$, which suggested that these genes play an important role in preventing accumulation of toxic intermediates that tether recombining DNA molecules together. Recently, experiments performed in srs $2 \Delta$ yeast strains, where a DSB induced by a site-specific HO-endonuclease was repaired by BIR allowed both visualization and subsequent investigation of toxic recombination intermediates [214]. Similar to other homologous recombination pathways, BIR is initiated by $5^{\prime}-3^{\prime}$ resection of a DSB end which then invades into the homologous template and begins synthesis that can copy $>100 \mathrm{~kb}$ of the template until the end of the chromosome [216, 217]. BIR proceeds by a migrating bubble where asynchrony between leading and lagging strand synthesis leads to accumulation of long regions of SSDNA [218]. These regions are stabilized by RPA, a sSDNA binding protein, along with Rad51, a strand exchange protein [219]. These protein-bound ssDNA located behind of BIR bubble can undergo unscheduled pairing with the template, which forms 
A.

I. $\quad \stackrel{5}{\prime}, \stackrel{\longrightarrow}{=}=$

No PCR product

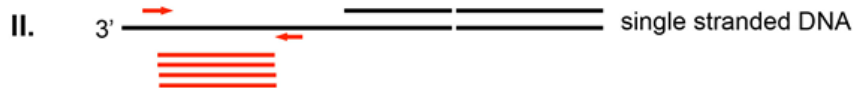

$\mathrm{PCR}$ product

Annealing oligonucleotide

III. $3^{\prime} \longrightarrow$ - $\longrightarrow$ - $=$ single stranded DNA

No PCR product

B.

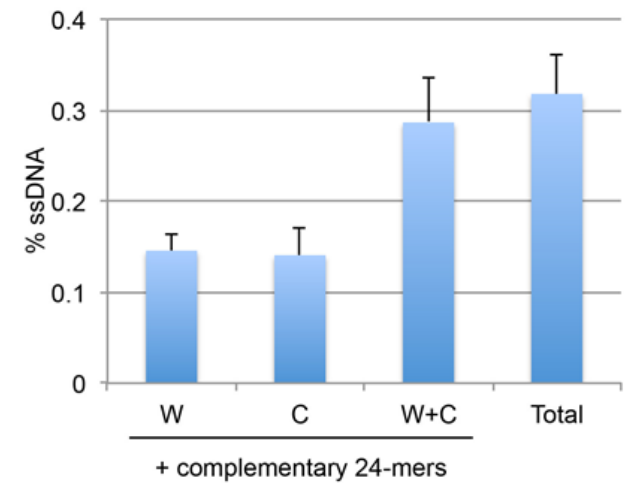

C.

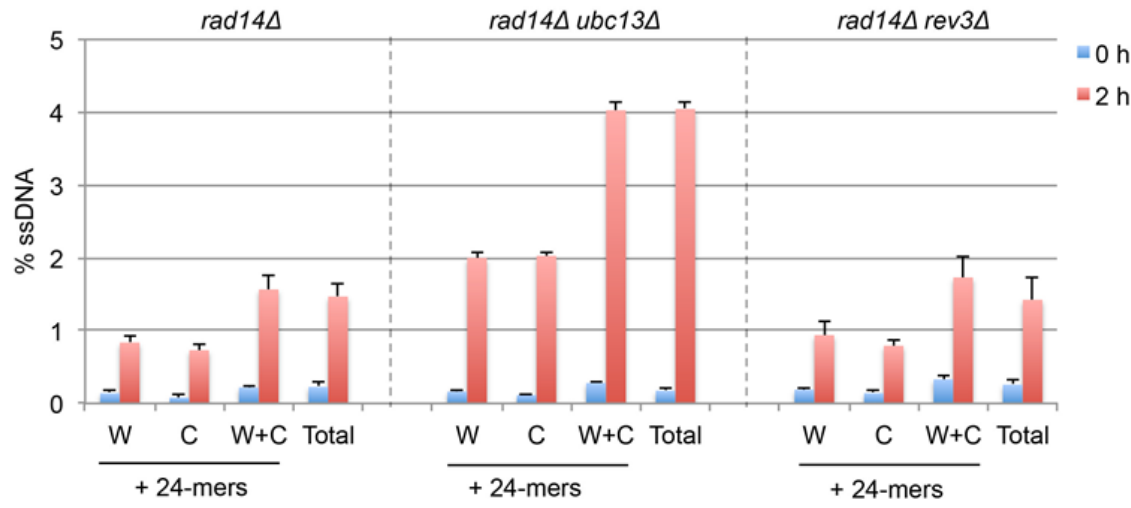

FIGURE 11: Schematic principles and determination of strand-specific ssDNA detection by restriction enzyme digestion and real-time PCR. (A) Detection of strand-specific ssDNA by real time PCR. i. Duplex DNA molecule is digested by BsaJ1 restriction enzyme. No PCR product will be generated using primers ( $a$ and $b$, red arrows) flanking the restriction enzyme recognition site; ii. ssDNA is resistant to enzyme cleavage and permits the formation of PCR products (red lines) flanking the restriction site using a set of primers (red arrows); iii. Addition of complementary oligonucleotides with 4 nucleotide mismatches at the 3 ' end (blue broken line) renders ssDNA cleaved by BsaJ1 and suppresses the formation of PCR products. (B) The level of total and strand-specific ssDNA at 2.1-kb right distal to the origin ARS305 in the genomic DNA isolated from G1-arrested RAD14+ cells. 24-mer complementary oligonucleotides to Watson or Crick strands were added to determine strand-specificity of ssDNA. Percent ssDNA (\% ssDNA) is calculated using the formula: $\% s s D N A=200 \times 2^{\Delta c t} /$

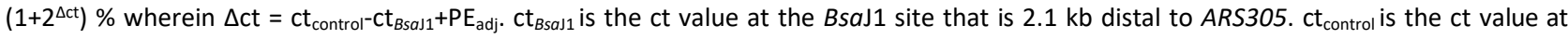
the locus 0.9-kb right distal to $A R S 305$, a control locus that does not contain a BsaJ1 restriction site; $\mathrm{PE}_{\mathrm{adj}}$ is a constant value adjusting the PCR efficiency difference between the primer sets for $0.9-\mathrm{kb}$ and $2.1-\mathrm{kb}$, which equals $\mathrm{ct}_{2.1 \mathrm{k}}-\mathrm{ct}_{0.9 \mathrm{k}}$ using undigested genomic DNA as the PCR template. The results are the average of three independent experiments + s.d.; (C) The level of total and strand-specific ssDNA at 2.1-kb

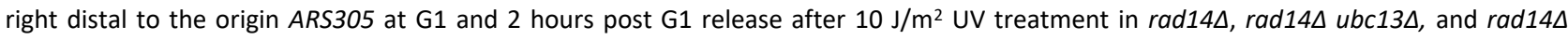
rev3 $\Delta$ cells. 24-mer complementary oligonucleotides to Watson (W) or Crick (C) strands were added to determine strand specificity of ssDNA. Percent ssDNA (\%ssDNA) is calculated as described in B above. The results are the average of three independent experiments \pm s.d. 
joint molecules that if not resolved become toxic recombination intermediates, joint molecules. It was demonstrated that accumulation of toxic joint molecules is counteracted by Srs 2 creating the opportunity to employ BIR experimental systems in $s r s 2 \Delta$ mutants to investigate genetics, kinetics of accumulation and disassembly, and the structure of toxic recombination intermediates.

The key features of the BIR experimental system (Figure 12A) used in these experiments [216] are:

1) A yeast $S$. cerevisiae strain that is disomic for chromosome III containing one full and one truncated copy of chromosome III. The truncated copy was created via replacement of the telomere proximal to the MATa region by LEU2 followed by a telomere sequence.

2) A galactose inducible DSB that is initiated at the $M A$ Ta locus of the truncated copy of Chr III, while the fulllength chromosome is refractory to cutting because it contains an uncleavable MAT $\alpha$-inc allele. Following the DSB, the broken truncated copy of Chr III invades into the fulllength copy and initiates BIR that proceeds for approximately $100 \mathrm{~kb}$. BIR is the predominant mechanism of repair in this system because almost all homology between the recipient and the donor is eliminated centromere distal to MAT [216].

Formation of toxic recombination intermediates during $\mathrm{BIR}$ in this system is assessed in srs $2 \Delta$ strains and can be followed by several different analyses, including genetic (viability) test, by measuring kinetics of DSB repair by contour-clamped homogenous electric field (CHEF) gel electrophoresis, two-dimension gel electrophoresis and electron microscopy. The first and second methods are the most straightforward and will be described here in more details.

To test cell viability following BIR induction, SRS2 and srs $2 \Delta$ cells are pre-grown in YEP-lactate media (not containing glucose, which is important for the efficient uptake and DSB induction by galactose) and then serially diluted and plated at an approximate concentration of 50 to 100 cells per plate on YEP media supplemented with $2 \%$ galactose (where DSBs are initiated and repaired) as well as on YEPD media (as a No-DSB control.) Following 3 to 5 days of incubation at $30^{\circ} \mathrm{C}$, the yeast colonies are counted and the viability is calculated by normalizing the number of colonyforming units (CFUs) on YEP-Gal by the CFU on YEPD. A minimum of three plating experiments are performed to calculate the average viability and standard deviation. Following initiation of BIR, the viability in $s r s 2 \Delta$ was approximately $30 \%$, which was significantly lower as compared to wild type cells ( 80\%) (Figure 12B) [214]. Such massive death detected in $\operatorname{srs} 2 \Delta$ mutants was unusual for this experimental system where the presence of two copies of Chr III, one of which remains unbroken, allows the cells to survive even in situations when the second, broken chromosome is left unrepaired and lost [216]. This loss of viability in $s r s 2 \Delta$ is indicative of the persistence of joint molecules that trap the donor and recipient molecules together.

Toxic intermediates are formed during BIR and can be detected by examining the kinetics of BIR which can be followed by separating BIR repair products from other chromosomes using CHEF gel electrophoresis. Using this method, the BIR repair product $(300 \mathrm{~kb})$ can be easily distinguished from the original (truncated) Chr III that, before $\mathrm{HO}$-induced DSB, is approximately 200-kb-long (Figure 12C) $[216,218]$. BIR repair time-course is performed by collecting $50 \mathrm{~mL}$ samples for CHEF before and after DSB induction. BIR typically takes $4 \mathrm{~h}$ for initiation and $5-10 \mathrm{hrs}$ for completion. Therefore, time points are collected every $2 \mathrm{~h}$ following BIR induction for a total of 8-10 hrs. Cells collected at each time point are spun down and embedded in $1 \%$ low melting agarose plugs after treatment with zymolyase. The resulting DNA plugs are then used for CHEF gel electrophoresis using the Bio-Rad-CHEF-DRII or other similar CHEF machines. When this experiment was performed in srs $2 \triangle$ and SRS2 strains, it was observed that the amount of BIR product (visualized by hybridization of the recipient chromosome with radioactively labeled $A D E 1$ probe) measured $8 \mathrm{~h}$ after DSB induction was nearly five-fold less abundant as compared to wild-type (Figure 12C, D) [214]. In addition, while the amount of template (donor) Chr. III molecule (visualized by hybridization with radioactively labeled $A D E 3$ probe) in wild-type cells remained constant throughout the course of BIR, in $s r s 2 \Delta$ it drastically decreased (Figure 12C, E). At $8 \mathrm{~h}$, the amount of the donor entering the gel was only $48 \%$ of the initial amount before DSB induction in $s r s 2 \Delta$ as compared to $96 \%$ in $S R S 2$. This decrease of donor molecules in the agarose gel in $\operatorname{srs} 2 \Delta$ is indicative of the accumulation recombination intermediates as branched DNA structures. These toxic branched structures can be further visualized by using twodimensional (2D) gel electrophoresis of Bglll or Kpnl restriction enzyme digested genomic DNA obtained from SRS2 and srs $2 \Delta$ cells undergoing BIR. Following 2D gel electrophoresis, DNA is transferred to a nylon membrane and is hybridized with radioactively labeled probes specific to various positions centromere-distal to the DSB located on Chr III (Figure 12F). Previously, this method was used to demonstrate that BIR is carried out by a migrating bubble, and SSDNA accumulates due to asynchronous synthesis of leading and lagging strands [218]. In srs $2 \Delta$, the bubble intermediate is barely detectable (Figure 12F) while another BIR intermediate becomes more prominent. This intermediate called "rubble" (Figure 12F) consists of heterogeneous DNA molecules resulting from toxic recombination that are more branched and heavier than those forming the bubble intermediate (see schematics in Figure 12G) [214]. The structure of individual molecules containing toxic intermediates can be further examined by electron microscopy, which allowed to determine that these toxic intermediates represent 3- and 4-way junctions formed by the unscheduled invasion of ssDNA accumulated during BIR and are positioned behind the BIR bubble [214].

When investigating the formation of toxic intermediates by $\mathrm{CHEF}$, these branched intermediates do not migrate inside the gel and remain in the well. They can be visualized in the well by hybridization with $A D E 1$-specific probe. However, since branched DNA from the well is diffi- 

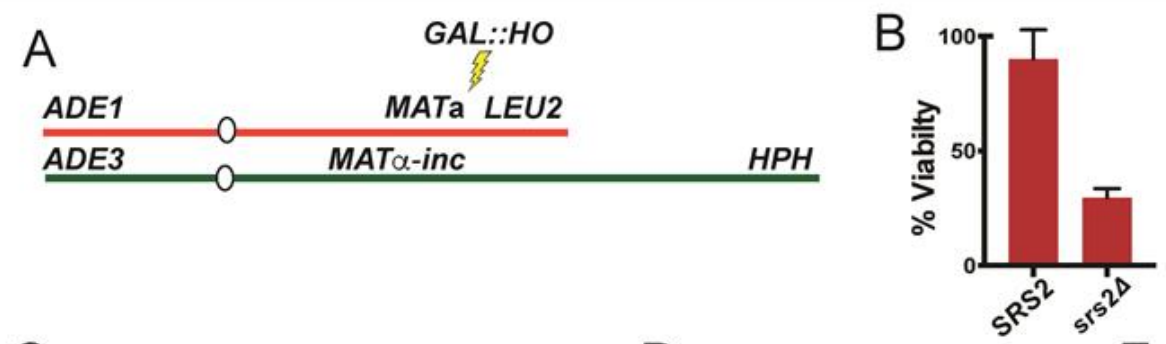

$\mathrm{C}_{0}$

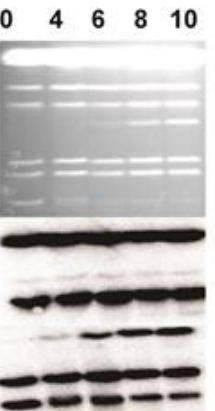

SRS2

F

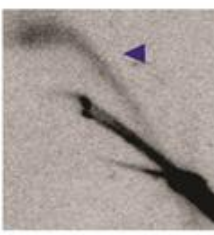

SRS2

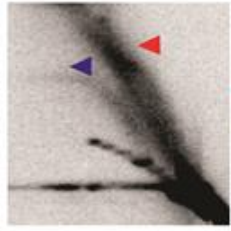

srs $2 \Delta$
D

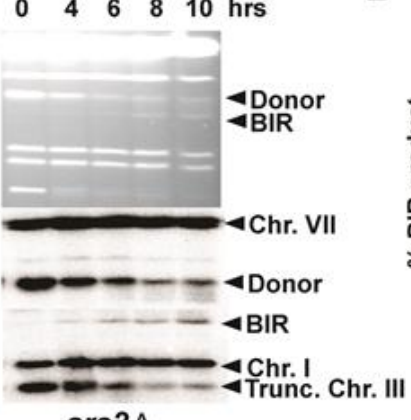

srs $2 \Delta$
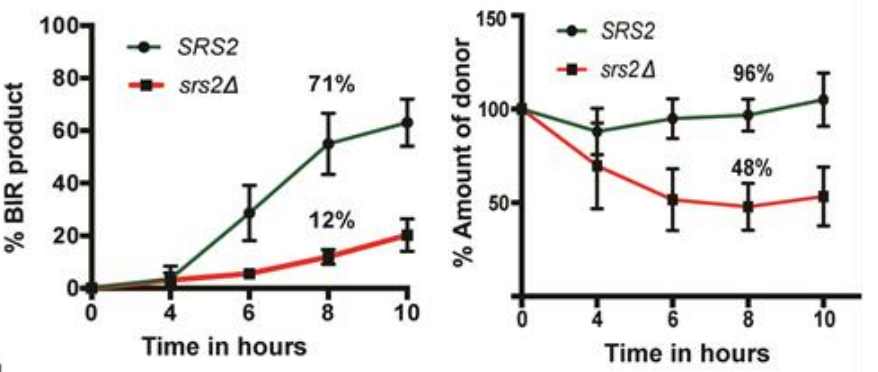

4 Rubble 4 Bubble

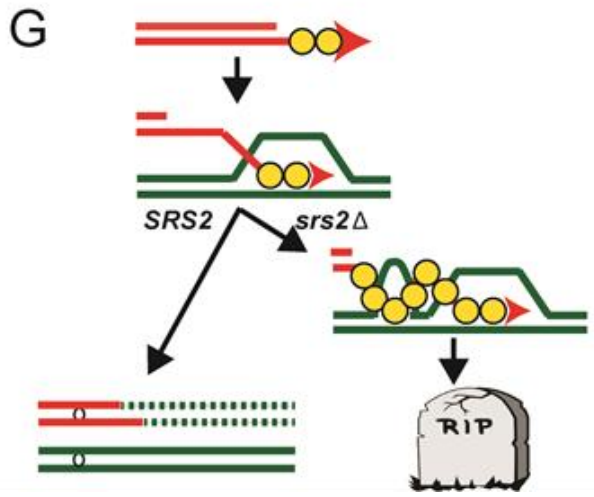

FIGURE 12: Detection of toxic recombination intermediates during BIR in yeast. (from data in Elango et al [214] published under open access under a Creative Commons Attribution 4.0 International License) (A) Experimental system to study BIR. BIR is initiated by DSB introduced by galactose-induced HO endonuclease at MATa locus in yeast disomic for Chr. III (B) Cell viability following DSB induction (\%). (C) BIR kinetics analyzed by CHEF gel using cells taken at indicated time points following DSB induction. Upper panel: CHEF gels stained with Ethidium Bromide. Subsequent panels below show Southern blot analysis using ADE1-specific, and ADE3-specific probes, hybridizing to the recipient and the donor chromosomes, respectively. (D) Quantification of BIR product. (E) Quantification of donor chromosome entering the gel. (F) 2D gel analysis of BIR intermediates in SRS2 and srs $2 \Delta$ at $7 \mathrm{~h}$ following DSB induction. Genomic DNA was digested with Bglll to detect intermediates at $24 \mathrm{~kb}$ position. Intermediates were detected using a probe specific to the $24 \mathrm{~kb}$ position of Chr III. Blue arrowheads denote bubble arc intermediates and red arrowhead denotes 'rubble' structure. (G) The schematics shows broken recipient chromosome (red) invading unbroken homologous donor (green). Repair DNA synthesis is initiated and progresses by a migrating bubble. In SRS2: successful completion of BIR with conservative inheritance of newly synthesized DNA. In srs $2 \Delta$ : formation of toxic joint molecules via unscheduled invasion of ssDNA located behind the BIR bubble into homologous chromosome leading to cell death.

cult for the transfer to the membrane, its quantification is complicated. In addition, accumulation of other branched intermediates takes place even in S-phase, and also even in $S R S 2$ cells, which further complicates its quantification. The transfer of branched DNA intermediates can be facilitated by additional UV Irradiation of the wells before transfer to the membrane. It should also be noted that the reduction of the amount of donor DNA entering the gel due to formation of branched intermediates might be difficult to detect later in the time-course (after 6-8 $\mathrm{h}$ following $\mathrm{HO}$ induction) because cells that successfully complete DSB repair (by BIR or gene conversion) continue to divide and eventually outcompete cells that stay arrested in their cell cycle due to accumulation of toxic repair intermediates. Also, toxic intermediates are likely formed in $\operatorname{srs} 2 \Delta$ (and possibly in some other mutants) during other recombination events [214]. However, it might be more difficult to detect them due to smaller chromosomal regions affected by these intermediates. 2D analysis of toxic recombination intermediates allows their visualization in a large cell popu- 
lation but does not allow analysis of the intricate details of their structure. Analysis of toxic intermediates by electron microscopy might provide a better resolution. However, identifying intermediates affecting only one of 17 chromosomes is difficult and tedious. A more detailed analysis of toxic recombination intermediates will require enrichment for the region of $\mathrm{Chr}$ III involved in BIR, which can be achieved by: (i) inserting a LacO array into the relevant region of chromosome III, (ii) performing a restriction digest with rare-cutting endonucleases, and (iii) conducting pulldown using immobilized LacR protein that recognizes and binds to the LacO sequence with high affinity [220].

Overall, the methods of analysis presented here allow for the detection of toxic recombination intermediates formed during BIR in srs $2 \Delta$ mutants. In the future, the same methods could be applied to the detection of such intermediates formed in other mutants, as well as in the context of other pathways involving recombination.

\section{Detection of hypermutable single-strand DNA formed in living yeast cells}

Resistance to DNA damage is in part assured by the double-stranded (ds) structure of DNA, which protects atoms of nitrogenous bases participating in hydrogen bonding. Moreover, the genomes of living cells can repair many thousands of simultaneously occurring DNA lesions without a trace, in many cases, due to the dsDNA structure, which provides an intact template for excision repair pathways. On the contrary, lesions in transient singlestranded (ss) intermediates of various DNA transactions (Figure 13) can easily be a source of mutations through error prone replication of the damaged strand. It was found in yeast that many kilobases of ssDNA formed by resection at DSBs or at uncapped telomeres, as well as by $B I R$, can be efficiently restored to dsDNA, even if the ssDNA contained dozens of lesions induced by ultraviolet light (UV), methyl methanesulfonate (MMS), chemical cytosine deamination by sulfites or endogenously induced abasic (AP) sites [221-225]. The recovery was assured by highly efficient TLS. Because TLS is error prone, many lesions in the transient ssDNA region were converted into closely spaced mutations (mutation clusters). Mutation density in a cluster exceed 1,000 - 10,000-fold overall mutation density in a genome, where lesions rarely occurred or were efficiently removed by error free NER or BER systems. A related source of lesions in a single DNA strand resulted in similar mutation types within that strand (strandcoordination). For example, after UV exposure, mostly pyrimidine bases were mutated in a single strand [221, 225]. Strand-coordinated clustering of mutations found in yeast prompted search for a similar phenomenon in genomes of human tumors, where such a clustering pattern was found in abundance in many cancer types [223, 226-230]. The most prominent cause of strand-coordinated clusters was identified as SSDNA specific APOBEC cytidine deaminases removing an amino group from cytosine which converts it to an uracil base. APOBECs are a part of the innate immunity system attacking retroviral and retrotransposon ssDNA intermediates. When these enzymes gain access to chro- mosomes they leave a trace of mutations, as long as the transient ssDNA persisted long enough to incur damage $[223,228,231]$. The exquisite specificity of APOBECs to deaminate cytosines result in exclusively mutated cytosines (or guanines) in the top strand reported in sequence data. Such C- or G-coordinated clusters could extend to many kilobases and contain dozens of mutations, all in $\mathrm{C}$ (or in G) nucleotides [223, 227, 229, 232]. The incidence of APOBEC-induced clusters indicated that long stretches of hypermutable ssDNA occur in human cancers and possibly in other types of cells and organisms. APOBEC enzymes were then used in yeast by several groups to explore the formation and genome-wide distribution of this unusual hypermutation substrate; long, persistent ssDNA.

By now, several different APOBEC enzymes expressed in yeast were used for this purpose: human $A P O B E C 3 A$, APOBEC3B, APOBEC3G and the lamprey APOBEC-like PmCDA1 deaminase [222, 227, 233-237]. In these experiments, APOBEC ORFs are usually expressed from a strong promoter, such as Gal1-10 or Tet. In wild type yeast, uracils formed by APOBEC cytidine deamination rapidly turn into AP sites by yeast uracil DNA glycosylase (Ung1). AP sites in a ssDNA template are copied by error prone TLS polymerases placing either an A or G across an AP site, thereby resulting in $C \rightarrow T$ or $C \rightarrow G$ mutations, respectively [238, 239]. AP sites can also break spontaneously or enzymatically, preventing the detection of long ssDNA based on mutagenesis. Thus, in order to improve detection of ssDNA stretches by APOBEC-induced cytosine deamination, UNG1 ORF can be deleted. During restoration to dsDNA, uracils in the template do not impede DNA polymerases and do not cause DNA breakage. Instead, all uracils are accurately copied with insertions of adenines resulting in $C \rightarrow T$ mutations. Also, since UNG1 defect blocks BER, mutations are generated even when there was a chance of BER to correct the damage using a second intact strand as a template (Figure 13D, E). Mutation detection is performed in isolates with selectable mutation reporters and/or by sequencing the entire yeast genome. This approach provided a useful tool for detecting different types of hypermutable ssDNA substrates described below.

\section{Uncapped telomeres (Figure 13A)}

Long ssDNA is formed at uncapped telomeres because they are recognized similar to the ends of DSBs and processed by $5^{\prime} \rightarrow 3^{\prime}$ end resection proteins [240]. In these studies, the telomere uncapping and the generation of long ssDNA overhangs were triggered by shifting temperature-sensitive yeast with a $c d c 13-1$ defect in the telomere capping protein to a non-permissive temperature of $37^{\circ} \mathrm{C}[221,222$ $225,227]$. Uncapping of telomeres triggered $5^{\prime} \rightarrow 3^{\prime}$ end resection and G2 cell cycle arrest. Cells can be kept in nonpermissive temperature for 6 hours in rich medium or for as long as $48 \mathrm{~h}$ in buffer, which allows sufficient time for $A P O B E C$ to deaminate multiple cytosines in the resulting stretch of ssDNA. Telomere capping is then restored when cells are moved back to a permissive temperature of $23^{\circ} \mathrm{C}$ followed by the restoration of dsDNA with adenines inserted opposite the uracils independent of a TLS system. Muta- 
tion reporters (different combinations of $L Y S 2, C A N 1$, URA3 and $A D E 2$ ORFs) placed in the vicinity of the left telomere of chromosome $\mathrm{V}$ showed over a 100 -fold increase in mutagenesis as compared to the same reporter placed in the middle of chromosome II, where end resection could not reach $[222,227]$. Sequencing of the reporter region followed by whole genome sequencing revealed strand biased C-coordinated (in right telomeres) or G-coordinated (in left telomeres) clustered mutagenesis consistent with long, up to $\sim 40 \mathrm{~kb}, 3^{\prime}$-overhangs generated by $5^{\prime} \rightarrow 3^{\prime}$ resection followed by multiple (up to 36 ) cytosine deaminations and resulting in $\mathrm{C} \rightarrow \mathrm{T}$ (or $\mathrm{G} \rightarrow \mathrm{A}$ ) mutations. The median density of mutations in a cluster was about 1.4 mutations/kb, indicating the size limitation for detection of a single stretch of persistent long ssDNA. Usually, clustered mutations were at several telomeres of a sequenced genome in addition to a cluster in the region of the selected reporter. So far, this approach has been applied only to cdc13-1 mutant yeast. In the future, it may become useful to detect problems with telomere capping in a variety of mutant strains carrying candidate genetic defects.

\section{Long bi-directional resection at DSBs (Figure 13B)}

Efficient recombinational repair of DSBs in yeast can occur with relatively short, as little as $150 \mathrm{bp}$, resected regions $[189,241]$. However, some breaks can undergo many kilobases of resection in the absence of a homologue and still be repairable with an oligonucleotide introduced into the cell by transformation [242]. Long bi-directional resection followed by repair using a sister chromatid was also suggested to explain the pattern of MMS-induced mutation clusters in yeast [223]. Hypermutable ssDNA formed by long bi-directional resection can be identified by switching the strand coordination pattern of mutation clusters. A single switch in strand coordination from $C$ to $G$ in a $5^{\prime} \rightarrow 3^{\prime}$ direction of the top strand (the strand reported in sequence data) is expected, because $5^{\prime} \rightarrow 3^{\prime}$ resection leaves single stranded DNA on the top strand to the left of the break and on the bottom strand to the right of the break. In the opposite case of single switch coordination where $G$ is followed by $C$, and/or in cases of multiple switches in strand coordination, bidirectional resection alone cannot explain these cluster patterns, and therefore they may be assigned to events involving more than a single break at the incidence of the cluster or to a cascade of breakage in the course of repair of a single initial break [243]. It is important to note that resection is not necessarily symmetric around a double-strand break [244]. If a DSB with long asymmetrical resection is repaired it could generate hypermutable ssDNA on only one side of a DSB which could result in completely C- or G- coordinated clusters similar to outcomes of BIR described below.

\section{Break-induced replication (Figure 13C)}

When only a single side of a break participates in repair by $B I R$, long hypermutable ssDNA is generated behind a BIR replication-like bubble upon initiation of DNA synthesis $[218,224]$. Importantly, BIR would generate a stretch of SSDNA in the same strand as long asymmetrical resection at the end of the break involved in BIR, thereby resulting in identical patterns of strand coordinated cluster. Nonswitching, completely C- or G-coordinated clusters were observed in yeast grown in the presence of APOBEC expression [233, 236]. A similar type of non-switching strand coordination conforming to the mutagenic specificity of MMS in SsDNA were reported in yeast growing in the presence of MMS or in association with BIR events that were triggered by a site-specific DSB repaired in the presence of MMS [224].

\section{Replication forks (Figure 13D)}

Long stretches of SSDNA are either rare or do not form in normal replication forks, however ssDNA specific mutagenesis can be detected by whole genome sequencing of yeast grown in the presence of APOBEC by preference of mutated cytosines over guanines in the lagging strand template [233]. This preference should be even more evident for mutations conforming to a known signature of APOBEC enzyme used in the experiment [245]. Hypothetical long-range uncoupling between copying leading and lagging strands in the presence of APOBEC expression would result in completely C- or G-coordinated clusters, depending on the strand delayed for copying as well as on fork direction. This pattern is the same as expected for one-end resection and/or BIR (Figure 13C). The orientation of clusters in relation to known replication origins, and the involvement of known replication defects would reveal uncoupling as an underlying mechanism for coordinated cluster formation.

\section{Transcription R-loops (Figure 13E)}

Because of the relatively small size of SsDNA formed in transcription associated R-loops, APOBEC mutagenesis is not expected to generate mutation clusters. Similar to replication forks, APOBEC mutagenesis in R-loops can be detected by a preference of mutations in cytosines over guanines in whole-genome sequenced APOBEC-mutagenized yeast, but in this case, preference should be for mutations in the non-transcribed versus the transcribed strand. APOBEC mutagenesis and the formation of mutation clusters biased towards non-transcribed strand was increased by inactivation of transcription initiation factor Sub1 [234]. APOBEC3B mutagenesis turned to be extremely efficient in non-transcribed strand of yeast tRNA genes [246]. These examples suggest that APOBEC mutagenesis may be a good additional tool for evaluating the scale of formation and persistence of R-loops in yeast.

While investigating mutagenesis associated with ssDNA, several potential limitations should be taken in account:

1) Overcoming high mutation load. It has long been known that haploid yeast strains with very high spontaneous or induced mutation rates, such as strains carrying a combination of defects in DNA polymerase proofreading and in mismatch repair, or in strains growing in the presence of the very strong mutagen 6-hydroxylaminopurine, hypermutated cells die out due to frequently occurring lethal mutations $[235,247]$. However, diploid strains show very high viability because the vast majority of lethal muta- 


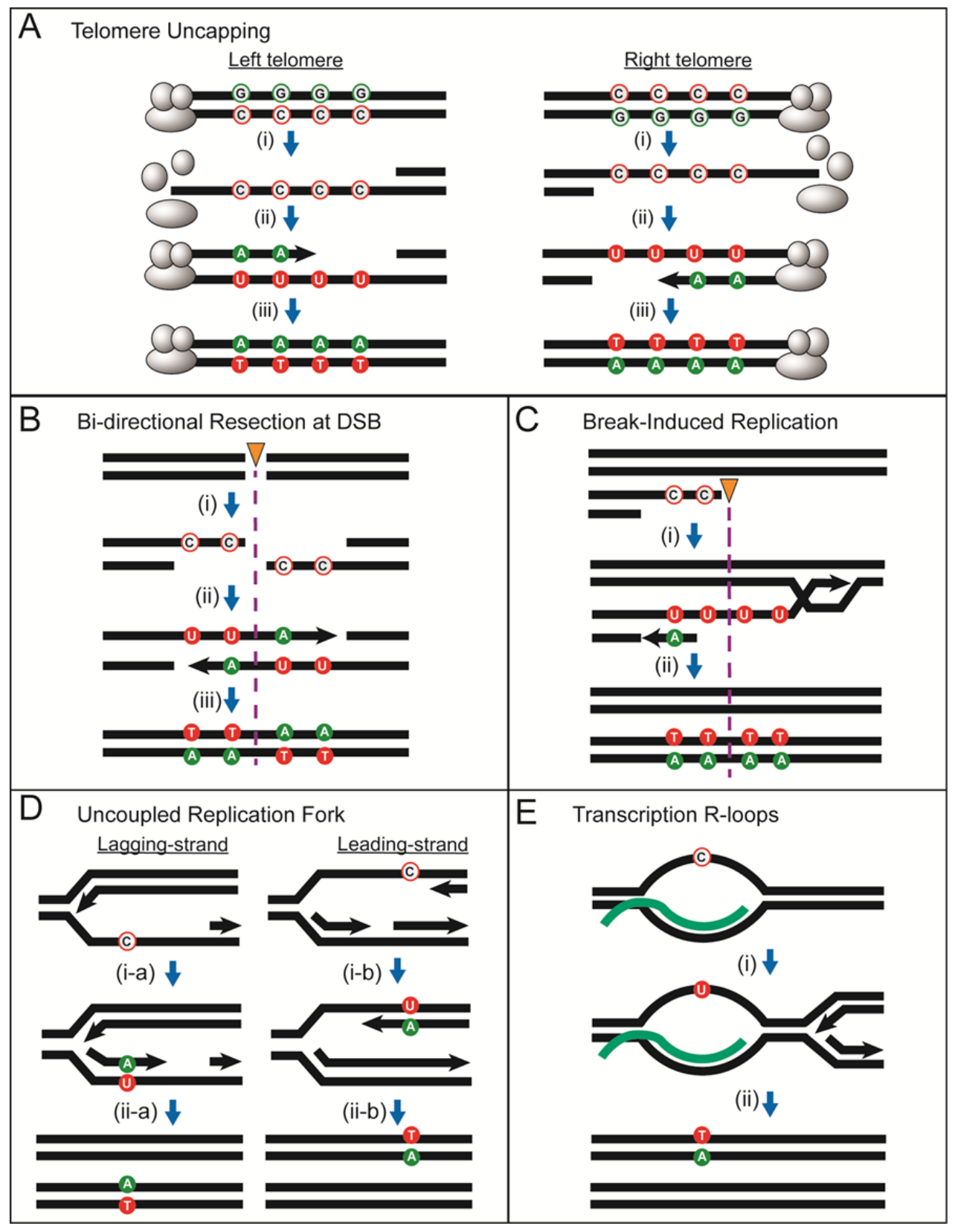

FIGURE 13: Cellular processes generating transient ssDNA vulnerable to hypermutation by APOBEC. The following symbols in the figure include: "unfilled green circles" = non-mutated guanines; "unfilled red circles" = non-mutated cytosines; "green-filled circles" = mutated guanines; "red-filled circles" = mutated cytosines; "black arrow heads" = 5 ' to 3 ' DNA synthesis; "orange triangle with purple dotted line" = position of double-strand break; " $\mathrm{C}$ " = cytosine; " $\mathrm{A}$ " = adenine; " $U$ " = uracil; " $T$ " = thymine. In all models, $C$ to $U$ changes are due to APOBEC cytosine deamination. (A) Long ssDNA generated at uncapped telomeres. (i) telomere uncapping triggers $5^{\prime}$ to $3^{\prime}$ end resection and $\mathrm{G} 2$ cell cycle arrest, generating long stretches of persistent ssDNA that provides a substrate for deamination of cytosines to uracils by APOBEC. (ii) the capping of telomeres is reestablished, initiating restoration of dsDNA with adenines inserted in the nascent DNA opposite the uracils. (iii) after the next round of replication, mutations are fixed, resulting in C:G to T:A transitions. Deamination of cytosines in the left telomeres result in G-coordinated clustered mutations on the top strand, while deamination of cytosines in the right telomeres result in Ccoordinated clustered mutations on the top strand. (B) ssDNA formed from bi-directional resection at a double-strand break (DSB). (i) 5' to $3^{\prime}$ end resection on both sides of a double strand break result in ssDNA in the top strand on the left-side of break and in ssDNA in the bottom strand on the right-side of the break. Cytosines in ssDNA on both sides of the break will be deaminated by APOBEC. (ii) and (iii) restoration of dsDNA using a sister chromatid or a homologous chromosome template followed by a round of replication will generate a single switch in strand coordination from C to $\mathrm{G}$ in a $5^{\prime} \rightarrow 3^{\prime}$ direction of the top strand. (C) ssDNA formed from the repair of a one-ended DNA break via BIR. (i) $5^{\prime}$ to $3^{\prime}$ end resection results in a $3^{\prime}$ overhang that invades a homologous template and initiates DNA synthesis that progresses via a migrating replication bubble that generates a long ssDNA tail behind the bubble. Asynchronous lagging strand synthesis initiates to restore DNA to double strand form resulting in C-coordinated clustered mutations. If BIR initiated repair from the opposite side of a break such that ssDNA was generated on the bottom strand, then this would result in a G-coordinated cluster. (D) Long ssDNA could form from uncoupling between replicating leading and lagging strands. (i-a) and (ii-a) stalling of lagging strand synthesis resulting in ssDNA formed on the bottom strand leading to a mutated guanine. ( $(\mathrm{i}-\mathrm{b})$ and (ii-b) stalling of leading strand synthesis resulting in ssDNA formed in the top strand leading to a mutated cytosine. If uncoupling leads to long stretches of ssDNA, coordinated cluster formation is possible. In both cases, the origin of replication (not shown) is on the left side. The polarity of mutated cytosines or guanines depends on the fork direction. (E) Small stretches of ssDNA in the non-transcribed strand at transcription Rloops. (i) and (ii) ssDNA in the non-transcribed strand results in genome-wide bias towards APOBEC-mutated cytosines in non-transcribed strands. If uncoupling leads to long stretches of ssDNA, coordinated cluster formation is possible. 
tions are recessive and therefore harmless in a heterozygote. Therefore, the use of diploid strains is advised in conditions when very high levels of APOBEC-induced genome-wide hypermutation is expected.

2) Non-uniform distribution of ssDNA amount in a population. If ssDNA formation is occurring in a small fraction of cells with excessive levels of replication, breakrepair or transcription problems (concerns even in systems with site-specific DSBs), then choosing candidates for whole-genome sequencing can be problematic. In these cases, the use of specially designed strains allowing selection of cells with clustered mutagenesis in a reporter can be helpful to reduce sequencing efforts [222, 223, 227]. Isolates with a mutated reporter can then be chosen for sequencing of either just the reporter area and/or the entire genome.

3) Low density of APOBEC-induced cytosine deamination. APOBECs deaminate less than 1 cytosine per 1000 nucleotides of ssDNA [227], therefore many ssDNA stretches can escape detection. Thus, the results can be interpreted only as a minimum estimate of the amount of long persistent ssDNA in the genome. On the other hand, this is close to the density of UV-, alkylation- (MMS) and chemical deamination- (bisulfite) induced mutagenic lesions in long SSDNA formed at DSBs and uncapped telomeres [221, 222, 225], which aids in predicting ssDNAassociated hypermutation capability in a variety of experimental settings.

4) Unknown continuity of ssDNA stretches. A low density of APOBEC mutagenesis would not allow the distinction between long stretches of continuous SsDNA versus relatively short ssDNA regions intercepted with dsDNA. This pitfall may be overcome in the future by developing hyperactive highly processive versions of APOBEC enzymes.

Overall, the patterns and organization of APOBECinduced clusters and single mutations in model studies may help us to understand APOBEC mutagenesis and SSDNA formation in cancers by analyzing the spectra, mutation signature and topography of mutation calls [223, $248,249]$. Knowledge about the scale and pattern of sSDNA formation is also important for understanding genome wide potential for hypermutation caused by a variety of endogenous and environmental factors other than APOBEC enzymes acting on ssDNA.

\section{CYTOLOGICAL ASSAYS TO MONITOR DNA REPAIR IN VIVO}

Cytological assays in living cells are based primarily of spectral variants of genetically encoded fluorescent proteins used to mark DNA repair proteins or chromosomal loci through in-frame fusion to DNA repair proteins or proteins that bind to DNA, respectively. Most microscope systems allow for simultaneous imaging of 3-4 different fluorophores, so that several DNA repair proteins and genetic loci can be imaging in the same cell. Some of these imaging techniques take advantage of photo-activation and photobleaching to turn on or off the fluorescence of specific fluorophores, which can be used to obtain information about the dynamics of single molecules in the cell.

The simplicity and amenability of microbial model organisms to genetic manipulation has made it possible to use the cytological approaches described in this section to provide mechanistic insight into the details of DNA repair pathways, including the order of events during repair, the duration of individual repair steps, the proteins and complexes involved in each step, and the correlation of these events with cell cycle stage and location of the lesion within the nuclear.

While often site-specific and inducible DNA lesions are used in population-based assays, the cytological assays can be designed to monitor spontaneous DNA damage caused by endogenous processes in the cell under various physiological conditions. This possibility can allow for a better understanding of natural processes of mutagenesis and genome dynamics.

In this section, cytological assays in $E$. coli and yeast designed to detect DNA damage and monitor repair in living cells are described (Box $\mathbf{3}$ ).

\section{In vivo probes to detect DNA repair intermediates} Engineered proteins for quantification and trapping of DNA $D S B s$ in living bacterial and mammalian cells

DNA DSBs are transient intermediate structures in genome-instability reactions, including activation of DNAdamage responses and repair. DSBs also occur in programmed developmental processes including meiotic recombination and antibody gene diversification in mammals. Though DSBs can be generated and engineered experimentally for study of repair, an important void in DNA biology has been accurate quantification and detection of the amounts, sources, and frequencies of spontaneous, endogenously generated DSBs in cells: i.e. how important are DSBs relative to other DNA-damage types in genome instability and routine DNA-repair reactions? Several methods used to study DSBs in cells and in genomes have been either non-specific, recognizing various DNA damage substrates or surrogate markers, like single-stranded DNA or DNA-damage-signaling events, rather than specifically DSB ends. There are only two demonstrated highly specific tools for detection of DSBs in bacterial cells, one also useful in mammalian cell culture: (i) genetic studies that compare recBCD-null-mutant with wild-type $E$. coli (or other bacterial) cells-phenotype differences indicate a role for DSBs at some stage of the process underlying the phenotype observed; and (ii) engineered fluorescent fusion proteins based on the DSB-end specific binding protein Gam of bacteriophage $\mathrm{Mu}$, which we developed [250]. To our knowledge there is no strictly DSB-end-specific reagent reported yet in yeast models. Because DNA structure is conserved, GamGFP and other Gam-fluorescent proteins (GamFPs) work to label and "trap" DSB ends, blocking repair and other proteins' action on them, when produced in E. coli, other bacteria, and mammalian cells. 


\section{BOX 3: CYTOLOGICAL ASSAYS TO MONITOR DNA REPAIR IN VIVO}

Detection of double-strand breaks in living cells | Use of methods to trap and monitor fluorescent proteins that bind to DSB ends. Can be applied to bacterial and mammalian cells.

Detection of Holliday junctions in living cells | Use of methods to trap and monitor fluorescent proteins that bind to Holliday junctions..

DNA repair protein dynamics through single-particle tracking | Tracks motion of DNA repair proteins to determine diffusion as well as kinetics and spatial distribution of DNA binding.

FRAP measurements of DNA repair centers | Methods of fluorescence redistribution after photobleaching to determine dynamic behavior of repair proteins.

Visualization of chromatin dynamics in cells | Methods to monitor chromosome dynamics following double-strand breaks. The method can follow chromosomal locus subnuclear mobility and relocalization. Mobility can be quantified using a mean-square displacement calculation.

Replication fork stalling | Use of a fluorescently-tagged locus can monitor stalling of replication forks at a specific replication fork barrier. Resolution of a blocked fork in time and space can be determined.

Double-strand break formation and resection methods | DSB end resection in vivo can be monitored by loss of a fluorescence signal located adjacent to a DNA break. Position and dynamics of the break can be studied simultaneously with break end resection.

\section{Conceptual description of the method}

We engineered gene fusions to encode the phage Mu Gam protein, a double-strand-end specific DNA-binding protein, fused to various fluorescent proteins to create GamGFP, GamMcherry, and others [250]. Mu Gam binds and protects DSB ends from degradation by nucleases and so is a natural DSB-end trap. GamGFP or GamMcherry are produced in $E$. coli from a chromosomally encoded regulatable cassette at a non-genic locus, the phage lambda attachment site. The doxycyline-inducible $\Delta a t t \lambda:: P_{N 25 t e t o g a m-g f p}$ cassette is transducible into other strains. In mammalian cells the GamFPs are engineered into plasmid vectors that are transfected into cells [250].

\section{Uses of GamFPs}

i) GamGFP binds and labels DSBs as fluorescent foci in single living bacterial cells

DSB Quantification. We showed that GamGFP detects DSBs generated site-specifically with I-Scel endonuclease cleavage of a chromosomal cutsite at each of several locations (e.g., Figure 14A-C) [250]. We used dose-response curves of ionizing radiation (IR) to estimate the efficiency of labeling of DSB ends based on physical quantification of DSBs at those doses in $E$. coli, and determined that GamGFP labels about $70 \%$ of DSBs expected to be present, and its dose-response was linear over a broad range [250] (Figure 14F).

One- and Two-ended DSBs. One-ended DSBs are generated by a single-strand endonucleolytic cleavage (ssDNA nick) in DNA then replication, which leads to a single DSB end when the fork collapses at the nick (Figure 14D, DSE). These were quantified similarly to two-ended DSBs [250]. Our data suggest that the two ends of two-ended DSBs form a single focus.
Cytogenetic mapping. Cytogenetic mapping of DSB locations in the $E$. coli chromosome showed that a site bound by a (red) TetR-mCherry repressor protein could be distinguished from GamGFP bound to an I-Scelendonuclease generated DSB 55kb or more away [250]. At $10 \mathrm{~kb}$ apart the foci overlapped. Thus, gross-level mapping of DSB ends with respect to a labeled site in the genome is possible using GamGFP in single living cells.

Quantification and origins of spontaneous DSBs reveal fewer than predicted. Using time-lapse microfluidic imaging, we quantified formation of spontaneous GamGFP DSB foci in $E$. coli at various growth rates [250]. We discovered that spontaneous GamGFP DSB foci form in a generationdependent manner, implicating DNA replication as a component of spontaneous DNA breakage. Because GamGFP traps DSB ends preventing repair (e.g., Figure 14G), once formed, the foci remained visible for at least $18 \mathrm{~h}$, and the cells with foci stopped dividing. The rate of spontaneous DSB formation detected with GamGFP was $0.015 \pm 0.006$ DSB foci per cell division [250], a rate in agreement within a factor of two with our previous quantification of spontaneous DSBs measured somewhat more indirectly as the RecBCD-dependent component of spontaneous SOSresponse-positive cells, measured using flow cytometry and a chromosomal SOS-activated promoter fused to $g f p$ also at a non-genic site: att $\lambda: \mathrm{P}_{\text {sulA }} g f p$ [251]. An mCherry version of this SOS-responsive DNA-damage detection cassette now also exists and is highly sensitive [252]. Importantly, with both GamGFP or SOS flow-cytometric assays, DSBs arise about 50-times less frequently than the once per $E$. coli cell division predicted from indirect estimates of DNA breakage by many previous authors. The standardization of both methods with known physically detected DSBs after IR or by I-Scel induction in E. coli [250, 
251], and the agreement between the different methods support these rates as accurate.

ii) Blocking DSB repair in E. coli

GamGFP blocks repair of DSBs causing phenotypes in E. coli like those of RecBCD-null mutant cells. These include sensitivity to UV light (Figure 14G) and large plaque formation by phage lambda recombination-defective and RecBCDinhibitor-defective red gam mutants [250]. GamGFP is thus useful for blocking the action of other proteins on DSB ends, including stopping repair mechanisms.

\section{iii) General Utility}

Several other studies of bacterial cells have used GamGFP successfully to block DNA repair [253] or detect DSBs as fluorescent foci [254-259] including our use of Gam to help quantify the efficiency of recognition of four-way DNA (Holliday) junctions in repair by RuvCDefGFP (RDG) [260], discussed in this paper.

iv) Mammalian Cells

Several groups have now used GamGFP and derivatives for detection of DSBs or inhibition of repair of DSB ends in mammalian cells [250, 261-263]. We first showed that GamFP fusion proteins label laser- and IR-induced DSBs as fluorescent foci, and block the early stages of DSB repair by inhibiting exonucleolytic resection that creates singlestranded DNA, detected by RAD51 binding and immunofluorescence [250]. Phage Mu Gam is an orthologue of mammalian (and bacterial) $\mathrm{Ku}$, and we found that the labeling of DSBs was more efficient in Ku-defective than wild-type mouse cells, implying that Ku may compete with GamFPs for DSB end binding [250]. We also used GamGFP in human and mouse cells to demonstrate that foci of gamma-H2AX histone staining really do label DSBs specifically [250]. However, another commonly used DSB/DNAdamage marker, 53BP1, did not label all GamGFP-labeled foci and appeared to label some sites other than GamGFPlabeled DSBs, calling into question the DSB-specificity of its labeling [250].

\section{Cautionary notes}

Quantification of DSBs in E. coli and other bacteria by GamFP foci:

Focus quantification challenge: when analyzing images taken from deconvolution microscopy, false positives may occur. Projected images of raw Z-stacked images may artificially generate images of cells with denser polar pixels, which may be mistakenly identified as foci. These artifacts can by filtered by applying a shape filter in the focus analysis software.

GamGFP foci are brighter than GamMcherry foci, in our hands. This suggests that the FP multimerization possible with GFP but not mCherry aids formation of detectable foci.

Cell sizes and numbers of chromosomes can vary between different genotypes. During an SOS DNA-damage response when cells "filament" because of inhibition of cell division, forming long cells with multiple chromosomes, cell length can be $>5$ times longer than normal [264]. Thus, normalizing numbers of foci/cell to DNA content is critical to accurate determination of the number of DSBs per chromosome or DNA amount in cells [250].

GamGFP is a "freeze-frame protein" trap. Because GamFPs bind DSB ends and inhibit their repair, cells with DNA damage are counterselected from growing populations because they cease to proliferate upon binding of GamFPs [250]. Care should be taken with extended growth with induction of GamFPs.

Protein levels of GamFPs must be comparable between different genotypes compared for focus levels to reflect DSB-formation rates. Because focus accumulation is cumulative-foci formed do not disappear, we showed [250] - similar lengths of induction time with similar inducer concentrations should be used.

Background. Both for E. coli and mammalian cells, the cell or nucleus becomes green when GamGFP is produced. Higher contrast between foci and other space than seen in live cells can be obtained in fixed cells, in which free GamGFP is removed. However live-cell imaging in E. coli is quantitative and straight forward [250].

Engineered proteins for quantification, trapping and genomic mapping of Holliday junctions in living bacterial cells Holliday junctions (HJs) are transient DNA reactionintermediate structures with four double-stranded arms. HJs are central intermediates in DNA recombinational repair, and also occur when replication is stalled and a fork "reverses." HJs are intermediates in, and can lead to, genome instability. Despite the centrality of HJs to DNA repair, replication, and genome-instability mechanisms, the ability to visualize, quantify, and map HJs in genomes of living cells has been limited, as has our understanding of the proteins that create, prevent, and remove them.

\section{Conceptual description of the method}

We engineered proteins that trap, map, and allow quantification of HJs in living E. coli cells and biochemically [260]. The new $\mathrm{HJ}$ visualization, quantification and mapping approaches are based on RuvCDefGFP (RDG), a GFP-tagged catalytically inactive version of the $E$. coli RuvC endonuclease. RuvC is the most four-way DNA-junction-specific protein known [265-267], and RDG can bind to but not cleave HJs [260].

We showed that RDG binds HJs and inhibits the action of other HJ-processing proteins on them both biochemically and in living cells [260]. That is, RDG "traps" HJs. RDG also forms quantifiable fluorescent foci that are correlated with HJs (representative images of foci in E. coli in Figure $\mathbf{1 4 H}-\mathrm{I}$ ), and is estimated to detect about half of HJs present in E. coli [260].

Moreover, the locations of HJs can be mapped in the E. coli genome using RDG chromatin immunoprecipitation followed by sequencing (ChIP-seq) [260]. We used RDG ChIP-seq to generate high-resolution maps of $\mathrm{HJ}$ footprints during repair of site-specific DSBs (Figure 14J) [260]. Using this powerful tool, we demonstrated the genome-scale directionality of homology-directed repair (HDR) of DSBs: 


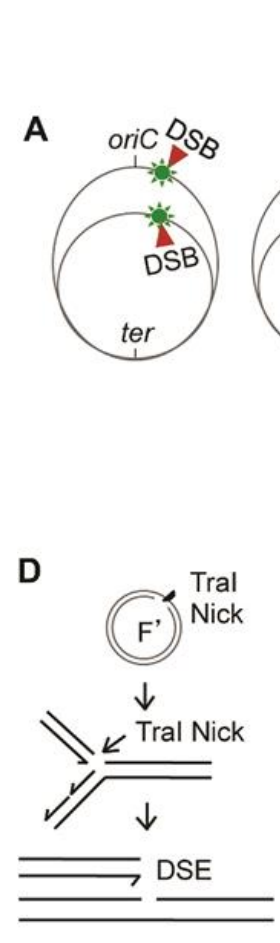

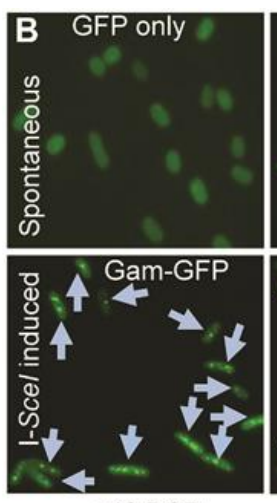

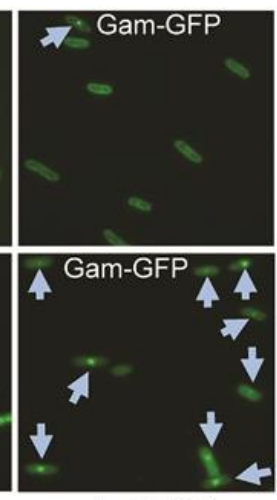

terC DSB

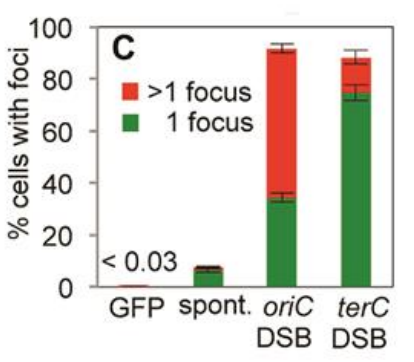

G
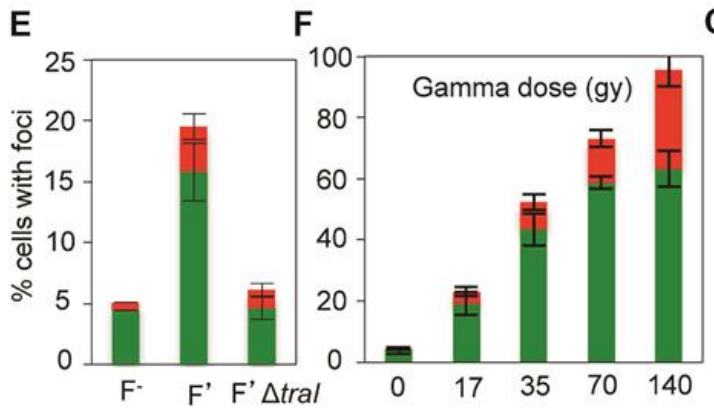

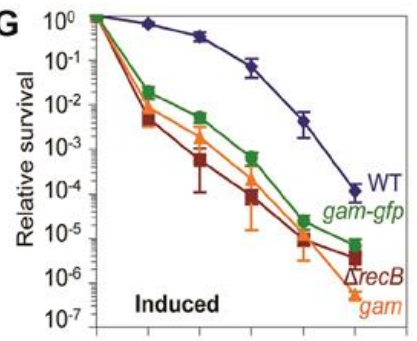

$\begin{array}{llllll}0 & 20 & 40 & 60 & 80 & 100\end{array}$
H

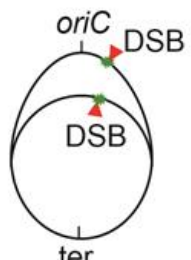

ter

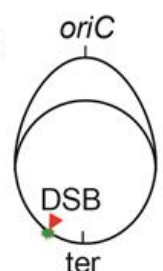

ter

I
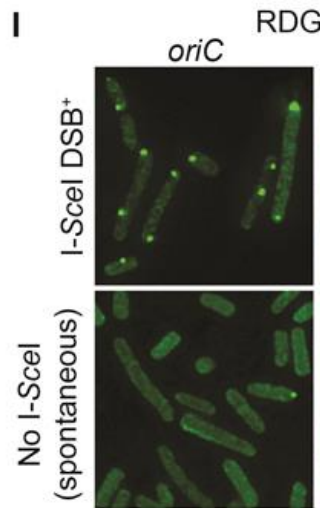
ter

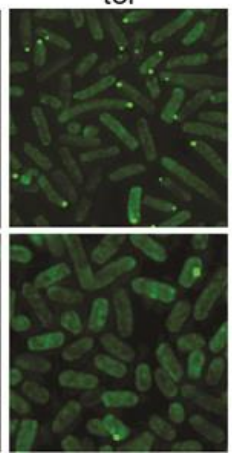

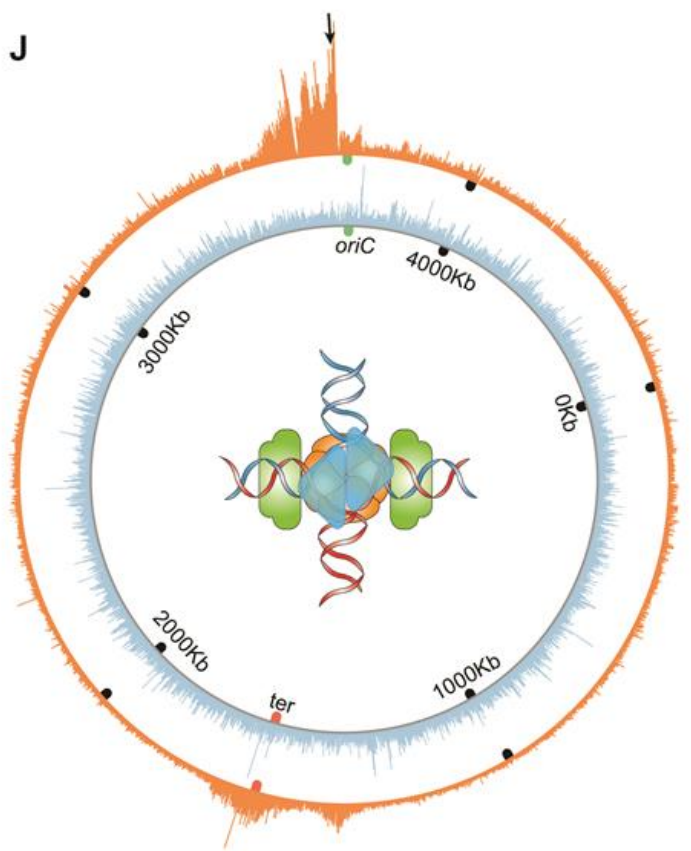

FIGURE 14: GamGFP and RDG quantify DSBs and HJs, map HJ footprints by ChIP-seq in living cells. (A-G) GamGFP foci quantify double-strand breaks (DSBs) in living cells and trap DSB ends causing DNA-repair deficiency. Data from [250]. (A) Strategy for E. coli chromosome cleavage with chromosomally encoded I-Scel ds endonuclease at engineered cut sites (red arrows). (B) Representative images of GamGFP foci without I-Scel cleavage (spontaneous, top row) and after I-Scel cleavage at sites either proximal to the replication origin (left column), or distal to the replication origin (right column). In replicating E. coli cells, there are more copies of the origin-proximal DNA than the origin-distal sequences, and more DSBs generated, and more GamGFP foci, with origin-proximal than -distal cleavage. (C) Numbers of GamGFP foci correspond with expected numbers of I-Scel-generated DSBs. Quantification of GamGFP focus data from multiple experiments diagrammed in parts A and B of this figure. (D) GamGFP recognizes one-ended DSBs generated by replication-fork collapse at an enzymatically-generated single-strand nick, made by the Tral single-strand endonuclease at its recognition sequence in a F' single-copy conjugative plasmid. (E) Quantification of one-ended DSBs generated by replication-fork collapse at the Tral-generated singlestrand nick in the $\mathrm{F}^{\prime}$ single-copy conjugative plasmid. F-, no plasmid. $\Delta$ tral, no Tral nuclease. (F) Linear dose response of GamGFP foci with gamma irradiation. With these data and the known number of DSBs per Gray of gamma in E. coli, we estimated the efficiency of GamGFP labeling of DSBs to be about 70\% (30\% of DSBs are not seen as foci). (G) GamGFP production blocks DSB repair, causing a sensitivity to ultraviolet (UV) light similar to that of DNA-repair-defective $\triangle$ recB null-mutant cells. (H-J) RDG foci represent HJs in living cells and RDG ChIP-seq maps HJ footprints. Data from [260]. (H) Strategy for E. coli chromosome cleavage with chromosomally encoded I-Sce I endonuclease at engineered cut sites (red arrows, DSB) (I) Representative images of RDG foci after I-Scel cleavage (top row) or spontaneous foci (bottom row), with cleavage near the replication origin (oriC) or terminus (ter). (J) HJ ChIP-seq at repairing DSBs shows directionality of DSB repair. The orange wheel shows the circular chromosome of $E$. coli bacteria. The spikes indicate where a molecular intermediate in DNA repair - four-way DNA junctions - accumulate near a reparable DSB in the genome (black downward arrow), and also at and after the replication terminus in the chromosome replication path. Blue, RDG ChIP-seq map of an uncleaved E. coli chromosome showing sites of spontaneous HJs. Center: diagram of HJ bound by RuvC (blue) assisted by E. coli RuvB (green) which stabilizes RuvC on HJs. 
that HJs accumulate at a site-specific DNA break undergoing repair and downstream from it in the chromosome replication path. These data provided experimental evidence for models of one-ended HR-DSB repair in the $E$. coli genome [268], in which the DSB-end attached to the replication origin primes processive break-induced replication that drags a HJ to the replication terminus $[260,269]$.

\section{Uses of HJ-trap proteins}

RDG binds and labels HJs in single living cells. There are many key outstanding questions in genome instability and replication that can be addressed using $\mathrm{HJ}$ trap proteins. Various assays measure HDR of, e.g., artificially induced DSBs, and have illuminated several HDR mechanisms. Yet, little is known about which mechanisms predominate spontaneously in somatic or vegetative cells. The HJ trap protein can detect and label HJs as fluorescent foci, thus enabling exploration of the following questions: What are the primary uses for and instigators of HDR in vegetative/somatic cells? How important is (what is the frequency of) HDR repair? RDG focus quantification combined with genetic analysis allowed us to address these questions [260]. We found that spontaneous HDR-HJs are replicationdependent, mostly resulting from non-DSB damage (ssDNA gaps) in vegetative $E$. coli. We also discovered the rates of formation of HDR-HJs, and that that their main sources are single-stranded DNA gaps, not DSBs. Recently, highthroughput microscopy techniques have been developed to allow genetic and chemical screening using RDG foci. In addition, the dynamics of $\mathrm{HJ}$ formation in living cells can be measured by tracking RDG foci in living cells by time-lapse imaging [260].

RDG for dissecting the stage(s) of HDR at which elusive HDR proteins act. Some proteins are required for HR repair but their primary role(s) are ambiguous. Trapping and quantifying HJs during HR repair allowed us to assess whether HDR proteins act before or after HJ formation. Timed expression of RDG and focus quantification can be used to dissect the stages at which recombination proteins act. Using this approach, we discovered a new "junctionguardian" role of $E$. coli RecQ DNA helicase: promoting HDR-HJs and preventing reversed-fork HJs. The-forkreversal-prevention role was also supported by bioinformatic data in human cancers for two human RecQ orthologs: BLM and RECQL4 [260].

ChIP-Seq approach combined with genetics. ChIP-Seq is a technique for genome-wide mapping of DNAinteracting proteins. We generated a high-resolution genome-wide map of the HR repair landscape upon I-Sce I induction of DSBs (Figure 14J). These first glimpses of the genomic footprints of HJs during DSB repair demonstrate a directionality of DSB repair along the chromosome not observed previously, with more HJs ori-distally than oriproximally of the DSB site. We have applied the ChIP-Seq technique with classical genetic analyses to understand DSB repair [260].

ChIP-seq peaks are prone to artifacts, so proper controls and careful experimental designs are essential for reliable interpretation of the results. Among these controls, the genetic controls, which we used in generating sitespecific DSB-induced HJ maps, give the most definitive answers.

\section{RDG: a candidate universal $\mathrm{HJ}$ trap}

RDG may potentially act as a universal $\mathrm{HJ}$ trap for organisms other than bacteria due to the conserved DNA structure of HJs. This has been supported by a recent study showing that RuvCDef binds t-loop generated HJs in human telomeres [270].

\section{Cautionary notes}

i) Quantification of HJs in E. coli as RDG foci:

Focus quantification challenge. When analyzing images taken from deconvolution microscopy, false positives can occur. Projected images of the raw Z-stacked images may artificially generate images of cells with denser polar pixels which may be mistakenly identified as foci. However usually these artifacts can by filtered by applying a shape filter in the focus analysis software.

Cell sizes and numbers of chromosomes can vary between different genotypes. For example, in some "filamented" cells- $E$. coli that have undergone an SOS DNAdamage response, with cell division block and thus have multiple chromosomes per cell-cell length can be $>5$ times longer than normal [264]. Thus, normalizing numbers of foci/cell to DNA content is critical to accurate determination of the number of HJs per chromosome or DNA amount in cells [260].

Expression levels of "trap" proteins must be comparable when comparing different $\mathrm{HJ}$ levels between genotypes. The easiest way to ensure this is measuring mean/median florescence intensity from the GFP in flow cytometry. In addition, prolonged trap-protein production is not recommended because trapping HJs can be toxic to cells, preventing proliferation [260].

Limited throughput. The numbers of cells that can be generated per image are still limited. Although a highthroughput-screening approach has been developed, only $10^{5}$ cells can be imagined per hour. Additionally, the large numbers of raw and deconvoluted images require significant data storage.

\section{ii) ChIP-Seq:}

Sources of antibody are critical to ChIP-Seq [271]. Although the commercial RuvC antibody we used (Santa Cruz Biotech) has been validated extensively, using antibodies from other sources to ChIP RDG requires further evaluation.

Low data quality such as low sequencing coverage will limit the sensitivity of ChIP signal [272]; so it is advisable that samples should have sufficient and similar sequencing depth.

ChIP-Seq displays profiles of bulk cell populations and does not provide single-cell resolution as foci do. Small peaks may be ambiguous due to high background signal; thus, use of multiple peak-calling algorithms is recommended for noise reduction. More importantly, genetic controls are the gold standard for interpreting peaks. To further enhance signal/noise ratio, it may be helpful to 
enrich target populations by cell sorting or other physical methods and to increase total volumes of cells.

\section{Methods to determine DNA repair protein dynamics in the cell \\ Single-particle tracking of nucleotide excision repair pro- teins inside living bacteria}

Single-particle tracking (SPT) combined with Photoactivated Localization Microscopy (sptPALM) provides an opportunity to perform complex molecular biology experiments inside living cells. By tracking the motion of DNA repair proteins in vivo, information can be extracted not only about their diffusion, but also about the kinetics and spatial distribution of DNA binding [273-275]. From a methodological point of view, a Total Internal Reflection Microscope equipped with a sensitive detector (usually an EMCCD camera [276]) is commonly used, allowing detection of individual fluorophores. The signal from individual emitters can be analysed and the position of a given fluorophore established with high accuracy (up to a single $\mathrm{nm}$ ) by Gaussian fitting. To determine the mobility of each fluorophore, the positions of individual molecules are linked into trajectories over multiple frames using a tracking algorithm [277].

Since most proteins in bacteria are present at a copy number, which is too high to resolve individual fluorophores, photoactivable fluorescent proteins (PAFPs i.e. PAmCherry) can be used, allowing the level of active fluorophores to be controlled (e.g. by varying the intensity of a $405 \mathrm{~nm}$ photoactivation laser) such that $\sim 1$ fluorophore is active per cell. This allows for the consecutive observation of all labelled proteins [273-275]. As an alternative to PAFPs, protein tags (i.e. HaloTag), which bind organic fluorophores provided externally can also be used. Once a functional fusion of the protein of interest to a fluorescent label has been constructed, the experiment can begin.

One example of the power of sptPALM was a study of the NER pathway in E. coli[278]. Fusions of UvrA and UvrB the proteins that initiate NER, to PAmCherry were introduced into the chromosome and their behaviour was studied in cells, before and after DNA lesions were induced by exposure to UV light. A movie was recorded with $15 \mathrm{~ms}$ frame rate and the positions of the fluorophores were localised in each frame and linked into trajectories. For each trajectory, an apparent diffusion coefficient was calculated based on the distance between subsequent localisations [277] (Figure 15A). Molecules bound to DNA showed a minimal change in position, whereas freely diffusing molecules showed large displacements between consecutive frames. The different populations of UvrA and UvrB molecules were quantified by fitting the distribution of apparent diffusion coefficients from tens of thousands of molecules (Figure 15B, C). UvrA was found to bind DNA stably for $\sim 3 \mathrm{~s}$ ( $40 \%$ of molecules) or interact with DNA transiently (low $\mathrm{ms}$ range) (Figure 15B). It was proposed that the transient protein-DNA binding is a part of the initial DNA search process, whereas longer binding is a damage verification step. On the other hand, UvrB showed very different behaviour, with the majority of UvrB molecules freely diffusing throughout the cytoplasm (Figure 15C). Cell exposure to UV light caused the recruitment of most UvrA and UvrB molecules to DNA ( $75 \%$ and $60 \%$ of molecules, respectively) to repair UV-induced lesions. These sptPALM experiments showed that, in contrast to some historical in vitro experiments, UvrA and UvrB rarely form a complex in solution; instead, UvrA is a DNA damage sensor, recruiting UvrB to DNA only after damage detection. Furthermore, by using catalytic mutants of UvrA, it was possible to decipher the roles of the two ATP binding sites present in each UvrA molecule, showing that cooperative action in both sites is necessary to recruit UvrB to DNA damage sites [278].

\section{Cautionary notes}

Previously, one of the key factors preventing the wider adoption of SPT and sptPALM in microbiology has been limited access to the sophisticated equipment and customwritten data analysis tools required for imaging single molecules. However, as these techniques increase in popularity, commercial single-molecule microscopes are becoming more affordable and data analysis tools for SPT are becoming more available [279], opening access to this technique for non-specialist users.

Last but not least, the critical step in all SPT experiments is the construction of a fusion between the protein of interest and the fluorescent tag. Occasionally, this results in an inactive protein. Therefore, the functionality of each fusion protein must be carefully verified. If the fusion is non-functional, the fluorescent tag can be placed at the other end of the protein or the length and nature of the linker can be adjusted to suppress the interference of the tag.

\section{Conclusion}

SPT is a powerful method, allowing biochemical experiments to be performed in the native environment of living cells. When combined with perturbations such as protein mutations, deletions, or overexpression it can be used to gain deep mechanistic understanding of molecular pathways, and it has been applied not just to the field of DNA repair $[274,275,280]$, but also to study the stringent response [281], transcription [273, 282] and translation [283]. SPT is becoming more and more popular, not only in the field of microbiology but also in the eukaryotic field [284]. Furthermore, the availability of user-friendly microscopes and analysis tools [285] will pave the way for STP to become a standard tool in any laboratory.

\section{Inter-foci fluorescence redistribution after photobleaching} (iFRAP) to measure dynamics of DNA repair centers DNA recombination and repair involve the assembly of large protein complexes at the site of DNA damage. The high local concentration of DNA repair enzymes at sites of DNA damage can be examined in living cells by GFP-tagging of the involved proteins, which results in the appearance of cytologically discernable foci [286]. These repair foci are highly dynamic in time and space with proteins associating and dissociating from the site of DNA damage on a time scale of seconds to minutes. The dynamic behavior of DNA 
A

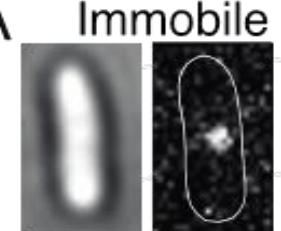

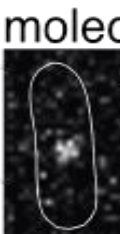
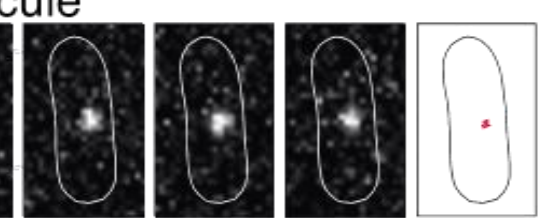

Mobile molecule
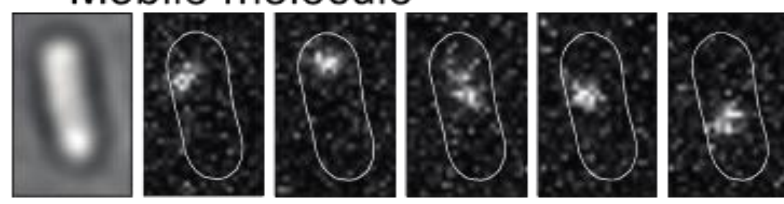

B

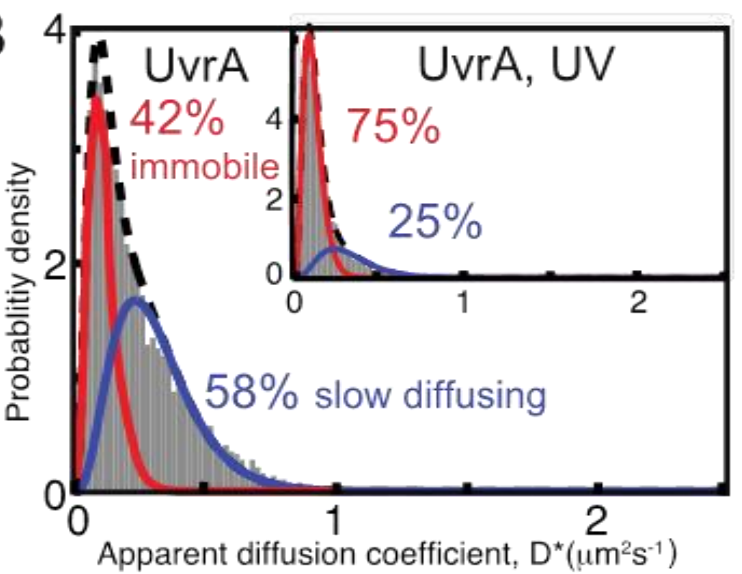

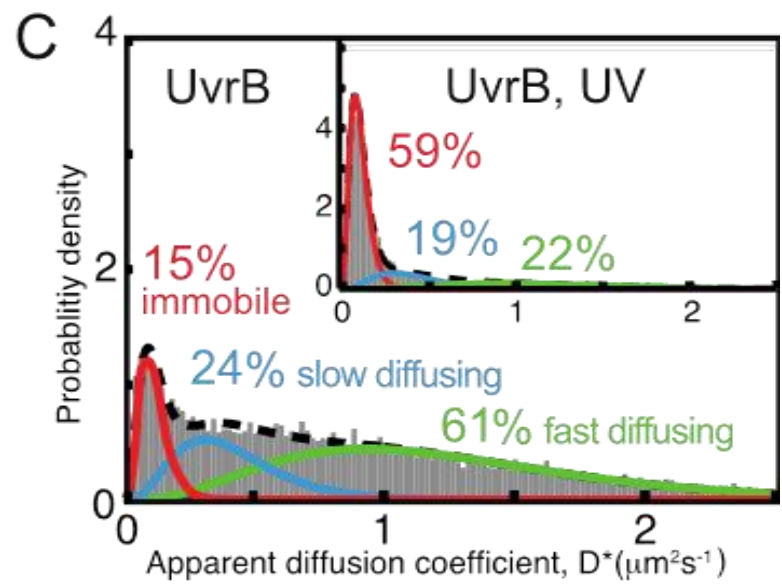

FIGURE 15: In vivo characterization of UvrA and UvrB proteins. (A) The example image of a single immobile UvrA-PAmCherry molecule localized and tracked at 15 ms exposures within five consecutive frames (top) and the example image of five consecutive frames showing fast diffusing UvrB-PAmCherry molecule (bottom). On the right, example cell is shown with multiple trajectories recorder for many individual UvrA molecules. (B) Distribution of apparent diffusion coefficients ( ${ }^{*}$ ) of tracked UvrA molecules, fitted with a two species model: first immobile, DNA-bound population ( $42 \%$ ) and second mobile population of slowly diffusing molecules ( $58 \%$ ). (Inset) The distribution of D* values of tracked UvrA molecules after exposure to $50 \mathrm{~J} \mathrm{~m}^{-2}$ ultraviolet light (UV). (C) Distribution of D* values of tracked UvrB molecules, fitted with a three species model established that $\sim 15 \%$ of UvrB molecules were immobile, $24 \%$ diffusing slowly and $\sim 61 \%$ fast diffusing. (Inset) The distribution of D* values of tracked UvrB molecules after exposure to ultraviolet light (UV).

recombination foci in vivo contrasts the very stable recombination complexes formed in vitro [287], suggesting that chaperones, remodelers and segregases may play a role in modulating the organization of the DNA recombination and repair machinery in vivo. The dynamic redistribution of proteins in living cells can be monitored by various photobleaching techniques such as fluorescence loss in photobleaching (FLIP) and fluorescence redistribution after photobleaching (FRAP), where fluorescently tagged proteins are locally photobleached by a brief exposure to intense laser light and the kinetics of redistribution of fluorescence due to diffusion of fluorophores from neighboring areas recorded. In the case of foci, this allows for the calculation of association/dissociation rates and mobile/immobile fractions to be quantified. In organisms with small size e.g. yeast and bacteria, these photobleaching techniques are challenged by the high rate of diffusion of most proteins, which results in complete redistribution within a time frame that is typically shorter than the time required for efficient photobleaching. For example, the mean squared displacement of GFP in the mammalian nucleus has been measured to $50 \mu \mathrm{m}^{2} / \mathrm{s}$ [288], which would allow it to diffuse across the $2 \mu \mathrm{m}$ of the yeast nucleus in approximately $0.01 \mathrm{~s}$. As a consequence, the photobleaching of a focus, which typically takes $0.5 \mathrm{~s}$, would lead to the simultaneous bleaching of the entire pool of soluble fluorescent protein in the nucleus. To address this challenge, we have established the inter-foci fluorescence redistribution after photobleaching (iFRAP) technique, where redistribution is monitored between two DNA repair foci within the same cell. In this technique, one focus is bleached, while the other focus serves as a pool for unbleached fluorescent molecules during the subsequent redistribution.

\section{Description of the iFRAP method}

In $S$. cerevisiae, the iFRAP method is best applied to recombinational repair foci after induction of approximately two-three DNA DSBsper cell. It is an advantage to use diploid cells, because their nuclei are slightly larger $(\varnothing=2.3$ $\mu \mathrm{m})$ than those of haploid cells $(\varnothing=1.8 \mu \mathrm{m})$ [289], making 
it easier to identify cells with two foci that are separated by more than $1 \mu \mathrm{m}$, which is the minimum distance required to photobleach one focus without collateral bleaching of the adjacent focus with most microscope configurations. Under standard time-lapse imaging conditions [290], twothree DSBs per diploid cell can be induced by treatment with $200 \mu \mathrm{g} / \mathrm{ml}$ Zeocin for $2 \mathrm{~h}$ or $30 \mathrm{~Gy}$ of ionizing radiation. After this treatment, $10-20 \%$ of the cells will have two foci of for example the Rad52 recombination mediator or the single-stranded DNA binding protein RPA (Replication protein A) [291], while most of the remaining cells have one focus. The low number of cells with two recombination foci is due to the clustering of multiple DSBs at a single recombination focus [292]. The iFRAP calculations are based on a time series of Z-stack images. We usually acquire one Zstack before photobleaching and nine stacks after photobleaching. Some fluorophores bleach more readily than others depending on the laser wavelength. We have good experience with bleaching of enhanced GFP [293] and yellow fluorescent protein (YFP, clone 10C) [294] using a $50 \mathrm{~mW} 488 \mathrm{~nm}$ laser.

After image acquisition, three fluorescence measurements are performed for each time point: the total nuclear fluorescence, the fluorescence of the bleached focus, and the fluorescence of the unbleached focus. Next, all fluorescence values are background-subtracted. Since image acquisition also causes a low level of photobleaching, the total nuclear fluorescence will continue to decrease in an exponential manner with each image acquisition. This bleach factor is determined by curve-fitting the total nuclear fluorescence for each time point after the laser event to an exponential decay function. The bleach factor allows for all fluorescence values after laser bleaching to be adjusted to compensate for the photobleaching caused by image acquisition. The maximum normalized fluorescence intensity $F_{\max }$ that the bleached focus can reach at the end of the experiment assuming $100 \%$ redistribution equals the total nuclear fluorescence after laser bleaching divided by the total nuclear fluorescence before laser bleaching. The recovery half-time $T_{1 / 2}$ and plateau fluorescence $F_{\text {end }}$ that the bleached focus approaches during redistribution is determined by curve-fitting using one-phase association non-linear regression. By comparison to the fluorescence of the bleached focus immediately after laser bleaching, $\mathrm{F}_{\text {start, }}$, the mobile protein fraction can be calculated as $\left(\mathrm{F}_{\text {end }}\right.$ $\left.F_{\text {start }}\right) /\left(F_{\max }-F_{\text {start }}\right)$ (Figure 16A).

In the presented examples (Figure 16B and C), the ssDNA binding protein Rfa1 rapidly turns over at the site of DNA damage and the Rfa1 molecules in foci are almost $100 \%$ mobile (Figure 16B). The turnover of Rfa1 is slower than the Rad52 recombination protein, but faster than the Rad51 recombinase [287], which could reflect the need to dissociate RPA from single-stranded DNA in order to nucleate a Rad51 filament. In comparison, the kinetochore subunit Mtw1 only slowly redistributes between the bleached cluster of kinetochores and the unbleached kinetochores (Figure 16C), and the majority of Mtw1 is immobile. This behaviour of Mtw1 is consistent with Mtw1 being a stable subunit of the MIND kinetochore subcomplex, which joins kinetochore subunits contacting DNA to those contacting microtubules [295].

\section{Cautionary notes}

The degree of laser photobleaching should be selected to allow for the most accurate quantitation. We have good experience with bleaching the target focus to around $10 \%$ of its original intensity, but for low abundant proteins such as Rad54, limiting the bleaching to $20 \%$ of its original intensity improved the accuracy of the subsequent quantitation [287].

The fluorophore should be selected to avoid reversible photobleaching, which has been observed for some variants of GFP, especially at low pH [296]. This is easily controlled for by measuring the total nuclear fluorescence during the redistribution phase. No reversibility was observed for the enhanced GFP [293] and YFP [294] used in this study.

Post-acquisition fluorescence measurements are greatly facilitated if cells with foci of roughly equal size are selected for iFRAP. This is due to the greater accuracy associated with measuring the fluorescence intensity of relatively bright foci.

The advantage but also a potential limitation of the iFRAP method is that the rate of diffusion from one focus to another is a composite of the on-off rate at the unbleached focus and the on-off rate at the bleached focus, whereas the diffusion in solution for most proteins will be several orders of magnitude faster and therefore negligible. The iFRAP method does not allow us to independently determine the on-off rate at the unbleached focus and the on-off rate at the bleached focus. Thus, the on-off rates for the bleached protein may be different for the two foci of a cell. In the context of DNA repair, such differences could reflect different on-off rates at different stages of repair. Moreover, most genotoxic agents, including Zeocin, induce DNA damage at random in the genome and the biophysical properties of repair foci could be different at different genomic loci.

\section{Conclusion}

The iFRAP method offers a tool to study the dynamics of DNA repair foci in vivo in microorganisms and to uncover the role of molecular chaperones, segregases and remodelers in facilitating the assembly and disassembly of DNA repair machinery at sites of DNA damage. This will potentially help explain some of the differences observed between the in vitro biochemical properties and in vivo cytological behavior observed for DNA repair proteins [287].

\section{Methods to determine chromatin dynamics in the cell} Visualizing chromosomal dynamics following DNA DSB induction in yeast $S$. cerevisiae

DNA DSBs are the most deleterious type of DNA damage when they are not repaired by an error-free mechanism. Thus, understanding their behavior in living cells is of major importance. Using S. cerevisiae as model organism, where most of the DNA repair proteins are similar to those in humans, allows us to use genetic tools to characterize the 
A

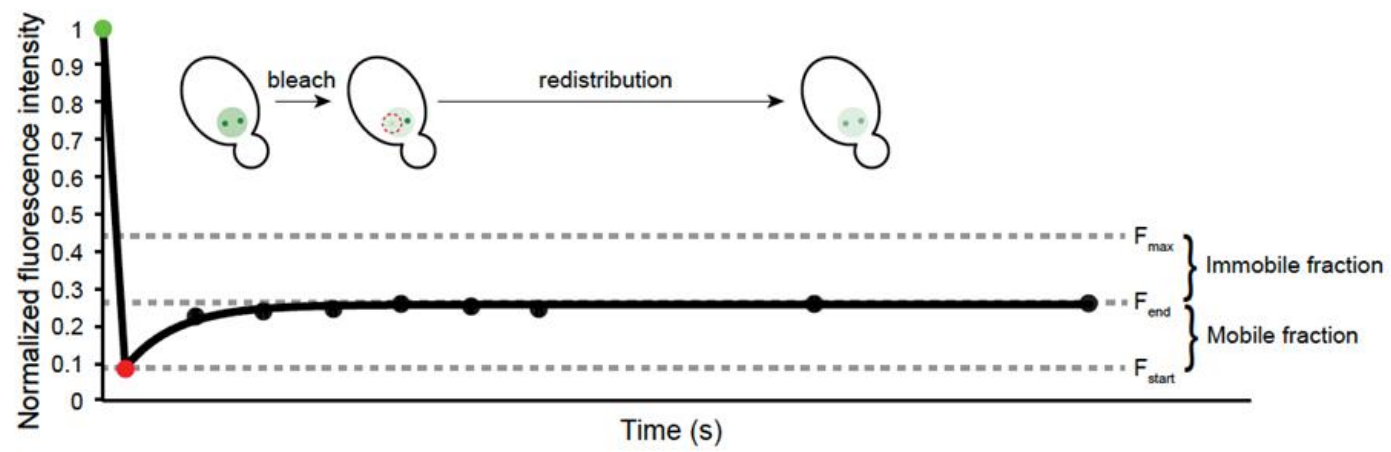

B
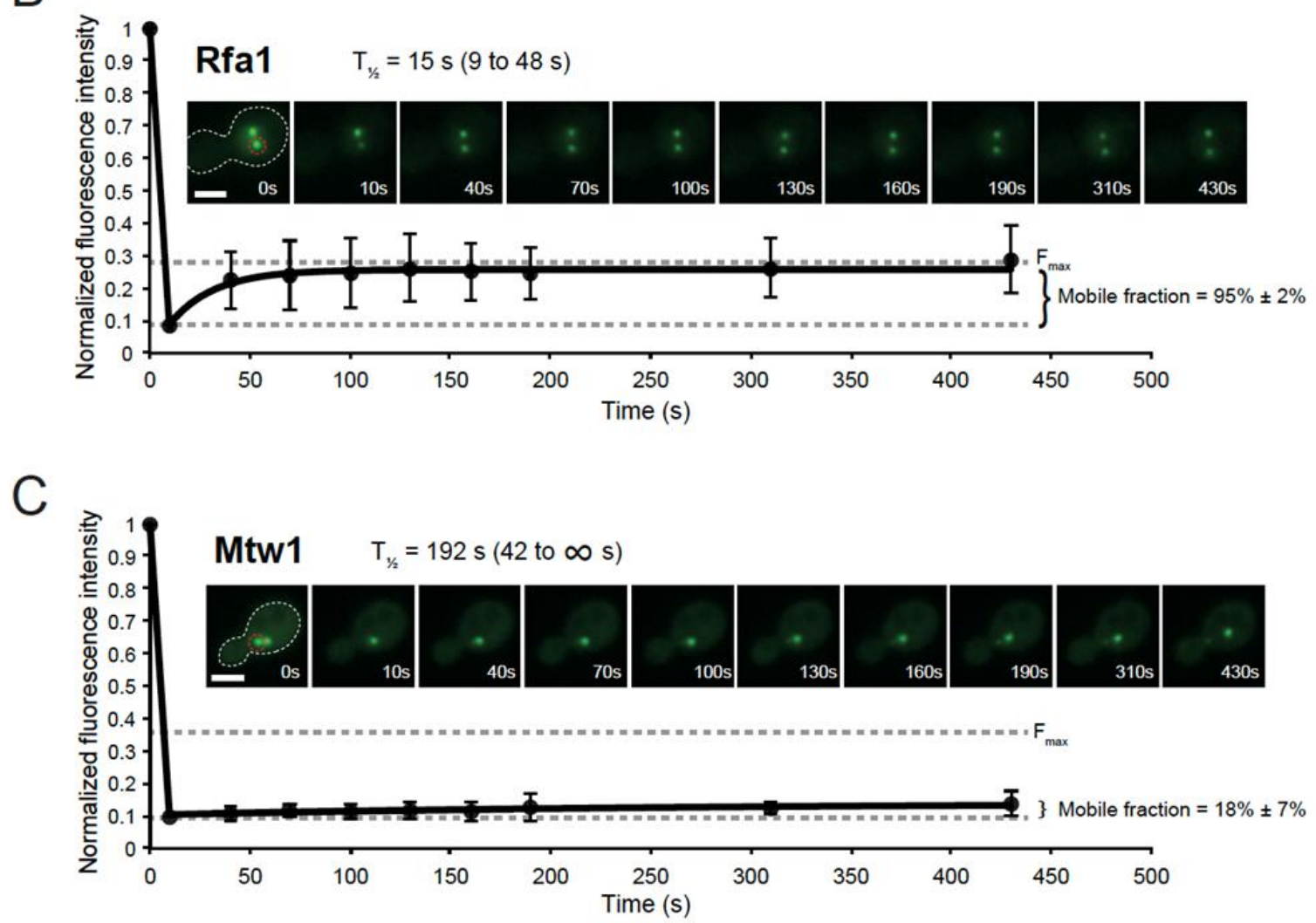

FIGURE 16: iFRAP analysis of Replication protein A and kinetochore subunit Mtw1. (A) Principle of iFRAP method. An image is acquired immediately before (green data point) and after (red data point) laser bleaching of one of two subnuclear foci (indicated by dashed red circle). The fluorescence intensity of the focus before bleaching is set to 1 and all subsequent fluorescence measurements are normalized accordingly. $F_{\text {start }}$ indicates the fluorescence intensity of the bleached focus immediately after laser bleaching. $F_{\text {end }}$ indicates the plateau fluorescence intensity that the bleached focus approaches during redistribution. $F_{\text {max }}$ indicated the maximum fluorescence intensity that the bleached focus can reach at the end of the experiment assuming $100 \%$ redistribution. The mobile protein fraction can be calculated as $\left(F_{\text {end }}-F_{\text {start }}\right) /\left(F_{\max }-F_{\text {start }}\right)$. (B and C) Cells with two foci were subjected to photobleaching of one focus (indicated by dashed red circle) at $t=$ $8 \mathrm{~s}$. Fluorescence redistribution was quantified at subsequent time points. The redistribution half-time $\left(\mathrm{T}_{1 / 2}\right)$ is indicated with $95 \%$ confidence intervals in parentheses. Scale bar, $3 \mu \mathrm{m}$. The protein mobile fraction is indicated \pm standard deviation. (B) RPA dynamically exchanges at DNA damage-induced foci. Cells expressing Rfa1-YFP (ML306) were grown in the presence of $200 \mu \mathrm{g} / \mathrm{ml}$ Zeocin for 2 hours to induce DNA repair foci. (C) Mtw1 is a stable component of the kinetochore. Cells expressing Mtw1-GFP [368] were arrested in M phase by treatment with $10 \mu \mathrm{g} / \mathrm{ml}$ nocodazole for 2 hours. One-phase association non-linear regression was performed using GraphPad Prism software. Error bars indicate SEM. 
properties of DNA DSBs. Using live microscopy, one can visualize the subnuclear localization of DSBs and examine how changes in position can influence the pathway of repair and/or repair efficiency. Three-dimensional tracking of a single DSB enables an in-depth characterization of the motion of the chromatin locus, and allows one to model it as a polymer fiber.

Determining chromosomal locus subnuclear position following DNA damage

It has been shown in budding yeast that when a DSB cannot be immediately repaired by recombination with its sister chromatid, it relocates to the nuclear periphery where it binds either the Nup84 nuclear pore subcomplex or an inner nuclear membrane SUN-domain protein, Mps3. The relocation and interaction with these two distinct sites has different effects on repair outcome, given that pore mutants and Mps3 mutants lead to different repair outcomes very differently. A three-zone technique for determining the position of damaged or undamaged loci [297, 298] has been useful for determining the precise positioning of the damaged locus relative to the nuclear envelope. One version of this imaging technique takes advantage of the bacterial Lac operator (lacO) sequence which binds the Lacl repressor fused to a green fluorescent protein (LaclGFP). One can then exploit a site-specific budding yeast endonuclease to create a single $\mathrm{HO}$ endonuclease cut at specific loci that are tagged by a lacO array. This permits a highly accurate determination of the subnuclear position of the induced DSB. The nuclear periphery is generally identified with a fluorescent tag (e.g., RFP) on the pore protein Nup49 (Figure 17A). Often the mating type locus, MAT, is tagged with lacO repeats and tracked, as it is the physiological target site of the $\mathrm{HO}$ endonuclease.

Method and data analysis. By placing the $\mathrm{HO}$ endonuclease under control of the GAL1 promoter, cleavage can be induced by the addition of $2 \%$ galactose for up to $2 \mathrm{~h}$. Cut efficiency is quantified by qPCR. Multi-stack images are acquired immediately, usually with a spinning disk confocal microscope. Spherical nuclei are needed for a proper statistical analysis of relative subnuclear positioning, thus G1 or early $S$ phase cells are usually analyzed, using the imageJ plugin Point Picker [298]. Briefly, the positions of the DSB and diameter of the nuclear pore-defined circle are determined in the same plane of focus. Using a pre-designed excel sheet, one can calculate the diameter of the nucleus, set this to one, and then determine a relative value for the distance between the periphery and the spot (Figure 17B). Hundreds of such measurements are taken for a given time point. To confirm that the site monitored is actually cleaved, it is often helpful to express a Rad52-YFP fusion, which will colocalize with the DSB.

Using a nuclear pore mutant nup133 $\Delta N$, which forces the majority of nuclear pores to form a large, single cluster at the nuclear periphery, one can accurately score the colocalization of the DSB with nuclear pores. On the other hand, DSB binding to Mps3 is best scored by Mps3 Chromatin Immunoprecipitation, because Mps3 gives only a weak fluorescence at the nuclear rim, apart from the spin- dle pole body. There may be additional perinuclear anchorage sites that remain to be characterized.

Cautionary notes. This technique relies on accurate dual-color imaging techniques, and it is crucial to take into account and correct for emission wavelength phase shifts. To score accurate distances, it is important only to score nuclei if the fluorescent spot is within the middle $50 \%$ of the Z-stack (not in the upper $25 \%$ or lower $25 \%$ of the nuclear sphere), due to the fact that resolution in the $Z$ direction is less good than in the $X-Y$ plane. One should not use DNA fluorescence as the boundary of the nucleus because the edge depends on thresholding, which is often subjective. Therefore, we recommend introducing a nuclear pore marker is essential for proper three-zone scoring. For a theoretical discussion of why the three-zone measurement is appropriate for such analyses, and the error inherent in the method, see our discussion of Cavalieri's principle and its validation through both empirical and theoretical troubleshooting [297].

Analyzing chromosomal locus mobility upon DNA damage It has been shown that chromatin dynamics increase upon DNA DSB induction [321, 322]. This probably improves repair efficiency by accelerating the homology search or by favoring relocation to a repair center. Using a similar cellular system for cut induction, as described above for the three-zone method of DSB localization (Figure 17A), a live cell tracking strategy has been developed to monitor DSB mobility over time.

Method and analysis. High speed time-lapse fluorescence microscopy allows one to track the Lacl-GFP tagged locus over time in living cells. In this case the center of the nucleus is used as a reference to correct for nuclear oscillations or translational movement of the entire field of vision. Different scales of image capture (i.e variation in the interval between image capture as well as the time of image capture) can be used. One system that has been useful has been the acquisition of single cell images every $80 \mathrm{~ms}$ taking Z-stacks of $200 \mathrm{~nm}$ for $1 \mathrm{~min}$ overall imaging time. Alternatively, longer times between stack can be introduced and image capture can be extended to 5 or $7 \mathrm{~min}$. One must always monitor for laser- or light-induced cellular damage, given that activation of a checkpoint response may bias subsequent measurements.

After deconvolution and $\mathrm{Z}$ projection, the ImageJ plugin Spot Tracker $2 D$ can be used to track the Lacl-GFP-tagged fluorescent locus relative to the Nup49-RFP-tagged nuclear envelope (Figure 17C). To compare locus position from one image stack to the next, one must align the nuclear centers through means of an idealized circle (the nuclear perimeter), and then determine the movement of the tracked locus within the aligned nuclear sphere. To quantify locus mobility, the mean square displacement of the fluorescent locus is calculated over time using a pre-designed excel sheet [299]. Mean-squared displacement is described in detail below.

Biophysical parameters derived from polymer model analysis can be applied to the spot trajectories to further characterize the motion. Briefly, the length of constraint Lc 
A

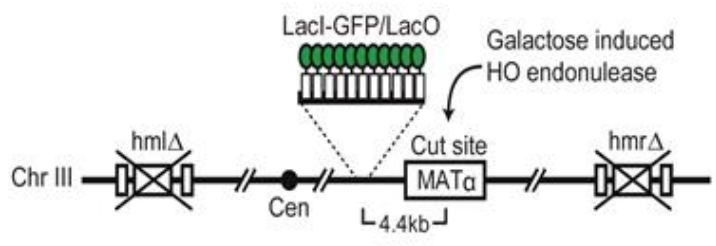

B

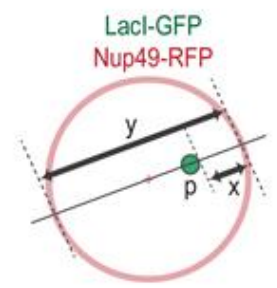

$\mathrm{x}=$ spot-pore distance $y=$ diameter $(2 x$ radius $)$ position $(p)=x /$ radius

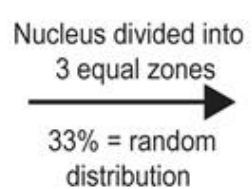

distribution

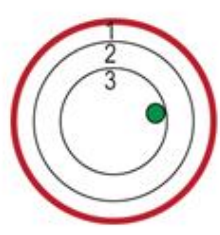

$p<0.184$ : Zone 1

$0.184<p<0.422$ : Zone 2 $p>0.422$ : Zone 3

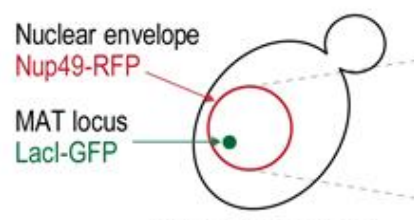

Budding yeast cell (S phase)
Lacl-GFP

Nup49-RFP

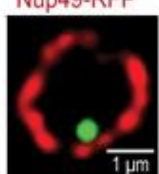

$\overline{1 \mu \mathrm{m}}$

c

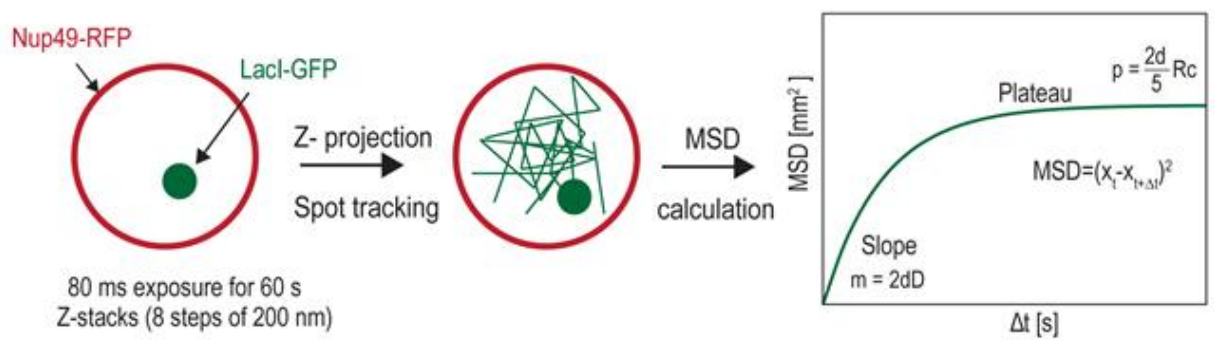

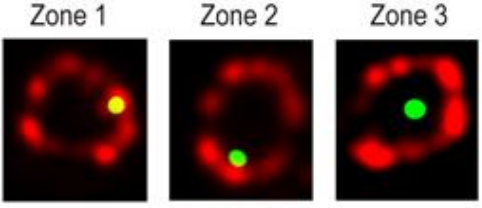

Lacl-GFP Nup49-RFP
Analysis

\begin{tabular}{|c|c|c|c|c|c|}
\hline \multicolumn{2}{|c|}{ Imaging } & \multicolumn{4}{|c|}{ Analysis } \\
\hline $\begin{array}{l}\text { Cut } \\
\text { induction } \\
(2 \mathrm{~h})\end{array}$ & $\begin{array}{c}80 \mathrm{~ms} \text { exposure } \\
\text { for } 60 \mathrm{~s} \\
\text { with Z-stacks }\end{array}$ & Deconvolution & $\begin{array}{l}\text { Nuclear alignment } \\
\text { (GFP-Nup49 signal) }\end{array}$ & $\begin{array}{l}\text { Z-projection } \\
\text { (Max. intensity) }\end{array}$ & $\begin{array}{c}\text { Spot tracking } \\
\text { (SpotTracker2D) } \\
\text { \& MSD calculation }\end{array}$ \\
\hline
\end{tabular}

FIGURE 17: Visualizing chromosomal dynamics at a DNA DSB in the yeast S. cerevisiae. (A) Schematic representation of the chromosomal locus tagging system. $\mathrm{HO}$ endonuclease is used to generate a single DSB at the mating type locus (MAT locus) that is tagged with a lacO array. The LacO array binds the Lacl repressor fused to a green fluorescent protein (Lacl-GFP). The nuclear envelope is visualized using a fluorescently tagged nuclear pore protein (Nup49-RFP). (B) Analyzing chromosomal locus position. Relative locus position ( $p$ ) is calculated by normalizing the distance pore-locus $(x)$ by the nuclear radius $(y / 2)$. The radial distances are then classified into three groups - Zone 1 (peripheral width $=0.184 \times$ nuclear radius $(r)$ ), 2 (middle width between $0.185 r$ and $0.422 r$ ) and 3 (central width=0.578r) - of equal surface. (C) Analyzing chromosomal locus mobility. Overview of the imaging procedure. Single cell, multi-stack images are acquired every $80 \mathrm{~ms}$ for $60 \mathrm{~s}$. After deconvolution and nuclear alignment, the 3D images are converted to 2D using a maximum z-projection. The tagged locus is tracked using SpotTracker2D and an absolute MSD calculation is applied.

measures the locus confinement in distance traveled, the effective diffusion coefficient Dc reflects its velocity, the effective spring coefficient Kc estimate the forces acting on this specific locus and the anomalous exponent $\alpha$ describe the nature of the motion [300].
Cautionary notes. The quantification of the trajectories relies directly on the imaging scale used. Recently, a study showed that changes in chromatin dynamics upon DNA damage depend on the tracking scale used [301]. It is therefore crucial to take into account this variation when choosing the time interval used for imaging. Basal levels of 
chromatin mobility (i.e without damage) vary during the cell cycle, and chromatin in G1-phase cells, is more mobile than in $S / G 2$. This has been attributed to the loading of cohesin in S/G2, to hold the two sister chromatids together after replication. Its removal and/or degradation allows an increase in the chromatin mobility [302]. Thus, to eliminate cell cycle -induced variability in movement, it is essential to compare mobility changes within the same cell cycle stage. For determining cell cycle stage accurately, see Neumann et al. 2006 [303].

Using S. cerevisiae as model organism allows us to accurately characterize chromatin dynamics following DNA damage. The use LacO/Lacl arrays for chromatin tagging enables scientists to determine specifically the position and dynamics of chromatin loci in response to DSBs. We and others have shown that chromatin dynamics and relocalization plays a central role in response to DNA damage, but how this impacts DNA repair efficiencies remains unclear.

\section{Quantifying the mobility of a chromosomal locus}

The dynamic organization of the genome is essential for many biological processes such as transcription, DNA repair, differentiation, etc. Indeed, the mode of diffusion of a chromosomal locus inside the nucleus dramatically affects the speed with which it interacts with surrounding molecules as well as with other chromosomal loci [304]. The mode of diffusion of a locus reveals aspects of how it explores nuclear space, how it deals with the obstacles it encounters, and how its movement relates to the organized structure of the nucleus. Thanks to the development of advanced microscopy techniques during the last ten years, it has become possible to measure and quantify chromatin mobility in living cells with unprecedented resolution.

\section{How to measure chromatin mobility}

The most common method to measure chromatin mobility consists of inserting a fluorescently tagged array at a given genomic locus and measuring its position through time. To tag a locus, repeated bacterial sequences, such as LacOperator ( $/ a c O)$ or Tet-Operator (tetO) arrays are inserted in the genome [305]. These arrays are bound by TetRepressor (TetR) and Lac-repressor protein (Lacl), which are fused to fluorescent proteins. The genomic locus is visible as a fluorescent spot by wide field microscopy and can be tracked over time. It should be noted that, in some cases, tightly bound Lacl and TetR repressors can create fragile sites or constitute a barrier of unknown penetrability to DNA processing enzymes [306-308]. To overcome these barriers, variants of Lacl, such as the Lacl** mutants [309], have been used as alternatives to bypass any possible bias. Another tagging method, consisting of the ParBINT DNA labelling system, has also been developed to fluorescently mark genomic loci [310].

To measure accurately chromatin motion, it is essential to correct for the motion of the nucleus during the acquisition of images. One method uses a fluorescent marker in the nuclear membrane to subtract the nuclear motion from the motion of the tagged genomic locus [311]. Alternately, it is possible to fluorescently mark a point that is relatively immobile in the nucleus during the time of the acquisition, such as the spindle pole body [312]. Finally, it is also possible to fluorescently mark two genomic loci and measure their relative positions over time. However, this method is used only when both loci have the same diffusion properties since it does not measure their motion independently [312, 313].

\section{How to quantify chromatin mobility}

Once the trajectory of a locus is determined, its diffusion properties are quantified by calculating its mean-square displacement (MSD) [314]. The MSD curve represents the amount of space a locus explores in the nucleus, and its shape reveals the nature of chromatin motion (Figure 18A). The time-averaged MSD of a single trajectory is calculated using the following equation:

$$
M S D(n-\Delta t)=\frac{1}{N-n} \sum_{i=1}^{\infty-n}\left[\left(x_{i+n}-x_{i}\right)^{2}+\left(y_{i+n}-y_{i}\right)^{2}+\left(z_{i+n}-z_{i}\right)^{2}\right]
$$

where $\mathrm{N}$ is the number of points in the trajectory, $(x, y, z)$ the coordinates of the locus in 3-dimensions.

In practice, chromatin dynamics is measured in several nuclei and individual MSD curves present some variability between cells. Averaged MSD curves among several cells (time-ensemble-averaged MSD) are usually calculated and fitted to characterize the type of diffusion of a locus in a specific condition. To understand the type of motion a chromatin locus undergoes, MSD curves are fitted using the different models presented below (illustrated in Figure 18A).

\section{Brownian diffusion}

When a particle freely diffuses, its MSD curve is linear with time and its motion is called "Brownian". In this case, the MSD follows:

$$
\operatorname{MSD}(\Delta \cdot t)=2 \cdot d \cdot D \cdot \Delta t
$$

where $d$ is the dimension of the movement, $D$ is the diffusion coefficient of the locus, and $\Delta t$ is the time interval.

\section{Sub-diffusive diffusion}

In living cells, DNA motion is often slower than Brownian diffusion and is called "sub-diffusive diffusion" [315]. Two types of sub-diffusive diffusion have been described: confined sub-diffusion and anomalous sub-diffusion.

Confined sub-diffusion. When a chromosomal locus stays confined inside a sub-volume of the nucleus, its motion is called confined sub-diffusion. The MSD exhibits a plateau [313] and follows the equation:

$$
M S D=R_{\infty}^{2}\left(1-e^{-2 \cdot d \cdot D \cdot t / R_{\infty}^{D}}\right)
$$

where $R_{\infty}$ is the measured plateau of the MSD, and $D$ is the diffusion coefficient of the locus. 
The confinement radius $(R c)$ of the motion is given by the relation: $R_{c}=R_{\infty} \sqrt{(d+2) / d}$, where $d$ is the dimension of the motion. The MSD curve starts to bend at time $t_{c}=R_{\infty}^{2} /(2 \cdot d \cdot D)$, representing the characteristic, equilibration time, after which the effect of boundaries appears.

Anomalous sub-diffusion. When the force or structure that restricts the motion is not a simple confinement but is modulated in time and space with scaling properties, the motion is called anomalous sub-diffusion $[315,316]$. In this case, sub-diffusive loci are constrained, but, unlike confined loci, they can diffuse without boundary and thus reach further targets if given enough time. For subdiffusive motion, the MSD exhibits a power law,

$$
M S D=A \cdot t^{\alpha}
$$

where $\alpha$, the anomalous exponent, is smaller than 1 .

The anomalous exponent $\alpha$ is linked to the degree of recurrence of DNA exploration, that is, the number of times a DNA locus reiteratively scans neighboring regions before reaching a distant position [317]. When $\alpha$ is small, the locus explores recurrently the same environment for a long time, while a large $\alpha$ indicates that the locus is able to explore new environments often. The anomalous diffusion coefficient A represents the amplitude of DNA motion; it is proportional to the diffusion coefficient only in the case of normal diffusion (when $\alpha=1$ ), which is rarely observed in biological systems [315].

\section{Limitations of experimental MSD and cautionary notes}

In practice, several artefacts can alter experimental MSDs. First, it is essential to take into account the effect of the inherent localization precision of the position of the spot. Localization precision can be divided into two contributions: i) the error in the determination of the accurate spot position, ii) the error due to the movement of the spot during the camera acquisition. Therefore, the finite localization precision adds a constant term to the MSD, which, if not properly accounted for, can limit or bias the exact quantification and interpretation of the MSD data. The exact analytic formula linking this constant term to the pointing accuracy and the motion blur have been calculated in the case of Brownian motion [318], and anomalous sub-diffusive motion [301].

Second, experimental MSDs are calculated from a finite trajectory length. Although each data point of the MSD is the result of an average over different times, at large time intervals, this average is performed over a smaller number of data points resulting in more scattered data and less statistical significance. To counteract this effect, the fit of MSD curves must be performed on a limited number of points to generate significant statistics. In practice, MSD curves are fitted using the first third of the MSD data points $[318,319]$. To increase statistical significance, MSD fits are often performed on the time-ensemble-averaged MSD (calculated by averaging several MSDs of individual trajectories). In this case, it is important to keep in mind that fitting the time-ensemble averaged MSD prevents the detection of heterogeneities in the population and provides ensemble-averaged behavior and diffusion parameters.

Finally, the MSD approach assumes that the kind of motion the locus undergoes is the same during the time of the acquisition. When diffusion is more complex, tracking chromatin motion at different time scales uncovers the different components of the motion.

\section{Multi-time scale tracking}

When studying the diffusion of a specific locus, the time scale at which the data are collected reflects the behavior of the locus at that specific time scale. Thus, this experiment allows the extraction of effective diffusion parameters that are true only at that specific scale. However, chromatin presents several levels of organization, which translates into different scales in chromatin mobility. To obtain a comprehensive understanding of chromatin diffusion, several studies have investigated chromatin mobility at multiple time-scales [301, 320]. Multi-scale analysis of the trajectories reveals the superimposition of different diffusion regimes, which may comprise different natures. For example, a locus can exhibit an anomalous diffusion of $A_{\text {micro, }}$ in a region that itself diffuses with a coefficient $A_{\text {macro }}$. Similarly, it is also possible that a locus exhibits anomalous diffusion at one time scale, but confined diffusion at a larger time scale. The MSD curves obtained at several times scales are represented altogether on log-log scale.

\section{Application in the context of DNA repair:}

Chromatin mobility is of particular interest in the context of DSB repair. Several studies have shown that chromatin mobility increases significantly in the presence of a DSB [321-324]. Figure 18B and 18C illustrate the motion of two homologous loci in diploid yeast cells, before and after the induction of a single DSB on one locus [312]. Using multiscale tracking following DNA damage, a recent study revealed the existence of several diffusion regimes that simultaneously drive chromatin motion [301]. In particular, chromatin exhibits increased mobility at large time scales, compared to undamaged cells, but reduced mobility at small time scales [301].

Altogether, quantifying chromatin mobility is a powerful tool to understand how chromatin explores nuclear space. Importantly, tracking and MSD analysis allows the measurement of apparent diffusion coefficients at a specific time scale. To gain a comprehensive view of chromatin diffusion, it is necessary to perform multi-scale tracking and multi-scale modeling to account for motion and rearrangements at different time scales.

\section{A fluorescently-tagged site-specific fork stalling assay: LacO-marked RTS1-RFB}

A fluorescence-based assay that is designed to track in vivo the fate of a single halted replication fork in the fission yeast S. pombe. Impediments to replication fork progression are a prevailing source of genome instability occurring during each cycle of cell division, contributing to the devel- 
A

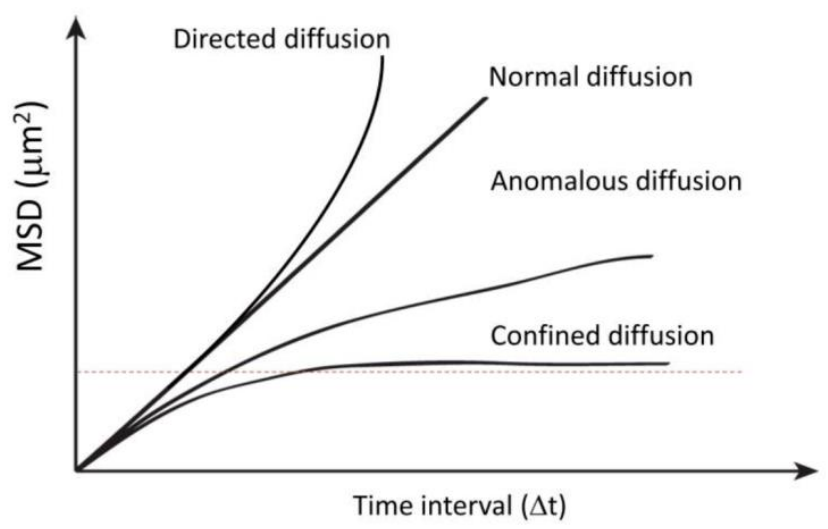

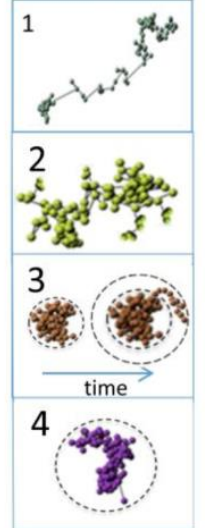

Directed diffusion

Brownian diffusion

Anomalous diffusion

Confined diffusion in a

limited space
B

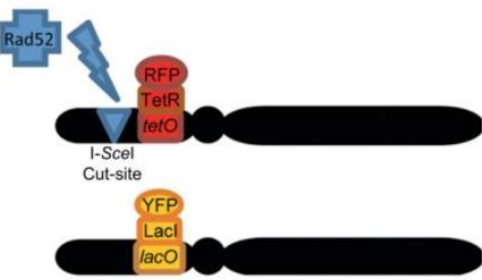

$\mathrm{C}$

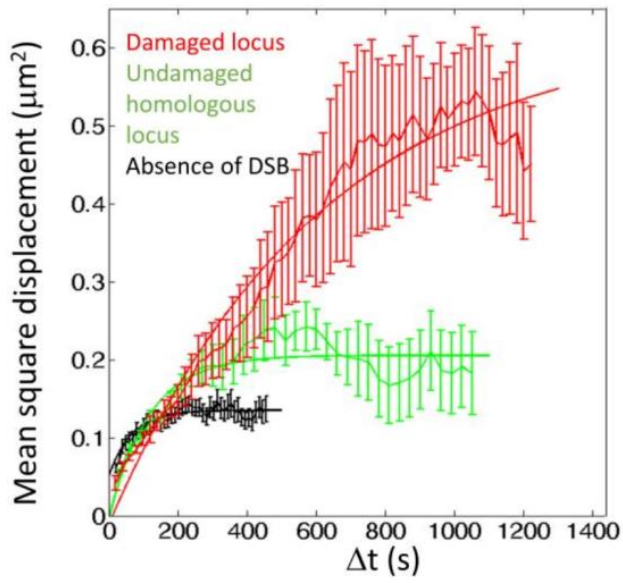

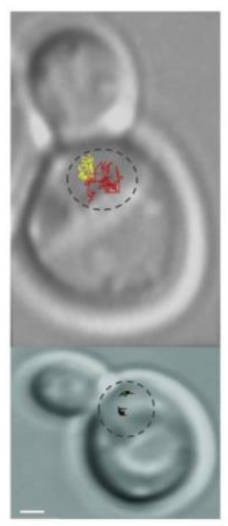

FIGURE 18: Studying chromatin mobility. (A) Left, theoretical Mean Square Displacement (MSD) curves for directive, Brownian, anomalous and confined diffusion. Right: corresponding typical trajectories for each mode of diffusion. (B) Experimental design to measure mobility at a chromosomal locus before and after DNA damage. A strain harboring two homologous and tagged loci (a tetO/TetR-RFP array inserted at the URA3 locus, and a lacO/Lacl-YFP array inserted at the homologous URA3 locus) contains, a single I-Scel cut-site located $4 \mathrm{~kb}$ from the tetO array. A galactose-inducible I-Scel inserted at the LYS2 locus allows regulated induction of a single DSB under galactose control. (C) Left, experimental MSD curves obtained from the diploid strain described in (B) Black: MSD curve of either tagged locus in the absence of DNA damage. Red: MSD curve of the tetO locus, after an I-Scel-induced DSB next to the tetO site (local mobility). Green: MSD curve of the homologous locus harboring a lacO array, after an I-Scel-induced DSB next to the tetO site (global mobility). Right: to illustrate the change in chromatin mobility, typical trajectories are shown (black trace: URA3 locus before DNA damage; red trace: damaged URA3 locus; yellow trace: undamaged URA3 homologue). The MSD curves are reproduced from [369].

opment of human diseases [325]. To address the consequences of disrupted replication forks on genome stability, site-specific fork stalling assays have been developed in yeast models and in mammalian cells [326-330]. These Replication Fork Barriers (RFBs) rely on secondary DNA structures or on the tight binding of a non-histone protein to a specific DNA sequence to act as a roadblock to fork progression. The $\sim 960$ bp RTS1 (for Replication Termination Site 1) sequence is a natural RFB from S. pombe, originally identified near the mat locus as an RFB necessary to fine-tune mating switching [331]. The RTS1-RFB was then integrated at ectopic sites, near a strong replication origin, to induce a polar roadblock to a single replisome (Figure 19A). Fork-arrest is mediated by the RTS1-bound protein Rtf1, the expression of which is regulated by the nmt41 promoter, allowing for the RTS1-RFB activity to be con- trolled ("OFF" and "ON"). Replication forks arrested at the RTS1-RFB are resolved in 20 minutes via the homologous recombination pathway which protects and restarts replication forks [328, 332]. Combining the RTS1-RFB with fluorescently marked locus-based approaches permits to follow in vivo the dynamic resolution of a single blocked fork in space and time [333]. Around 120 interrupted $\mathrm{LaCO}$ repeats were introduced $\sim 7 \mathrm{~Kb}$ away of the RTS1-RFB to create the LacO-marked RTS1-RFB locus (Figure 19A). LacO repeats are detected in vivo through the binding of the repressor Lacl whose $\mathrm{N}$ - and $\mathrm{C}$-terminals are fused to a fluorescent epitope (GFP or mCherry) and to a NLS, respectively. Snapshot and time-lapse fluorescently-based microscopy can be employed to visualize Lacl foci which mark the site of fork arrest in living cells using samples prepared in agarose pads or cells injected into dedicated 4-5 $\mu \mathrm{m}$ - 
thick poly-dimethyl-siloxane (PDMS) microfluidic chambers on glass coverslips. Detailed protocols for yeast cell imaging and sample preparations have been described [333, 334].

A first utility of the LacO-marked RTS1-RFB is to investigate the dynamic mobility of a single arrested replisome, respectively to surrounding nuclear compartments, by monitoring the Mean Square Displacement (MSD) of Lacl foci, as reported for other fluorescently-marked sitespecific DNA damage assays [298]. A second approach is the analysis of the recruitment of DNA repair factors tagged with a fluorescent epitope (taking care not to overlap their emission spectra with that of the tagged Lacl), to decipher the mechanisms of fork processing. For example, the fusion protein Ssb3-mCherry, one subunit of the trimeric single stranded DNA binding factor RPA (for Replication Protein $\mathrm{A}$ ) was used to analyze the formation and repair of single stranded DNA (ssDNA) at the RTS1-RFB during cell cycle progression (Figure 19B). A limited amount of Sphase cells showed RPA recruitment to Lacl foci, revealing the highly transient nature of ssDNA which is generated by the concerted action of several nucleases and then quickly resolved as the arrested fork is successfully restarted or rescued by an opposite fork [333]. In specific genetic backgrounds (in the absence of the recombinase Rad51 or its loader Rad52), numerous S-phase and G2 cells showed an extensive RPA recruitment to Lacl foci. These observations indicate that the RTS1-RFB accumulated large stretches of ssDNA (i.e. defined as unprotected forks) which are left unrepaired when cells enter mitosis, revealing that the arrested fork is neither restarted nor rescued by an opposite fork. Unprotected forks are then converted into RPApositive intertwined sister chromatids resembling unconventional mitotic bridges (i.e. refractory to classical DNA dyes), containing both ssDNA (marked by Ssb3-mcherry) and double stranded DNA (dsDNA) (labelled by GFP-Lacl which binds only double stranded $L a C O$ sequences) (Figure 19C). These approaches have revealed that unconventional mitotic bridges resembling human ultra-fine bridges are more complex than anticipated. In addition, the LacOmarked RTS1-RFB has proved to be sensitive enough to monitor the amount of RPA recruited at the arrested fork by quantifying the fluorescence intensity of Ssb3-mcherry being recruited to Lacl foci, after normalization by the nucleus area and background fluorescence intensity [335].

\section{Cautions}

Beyond already described issues when using LacO-arrays bound by Lacl as a fluorescently genomic tag, specific cautions are raised when combining with a site-specific RFB. The tight binding of Lacl to $\mathrm{LaCO}$ arrays acts as an intrinsic and non-polar RFB, resulting in mitotic bridges, chromosomes breakage and gene silencing [307-309]. Two approaches help to overcome this issue. First, a Lacl variant (called Lacl**) was reported to alleviate the impact of $L a$ cO-bound Lacl to fork progression while allowing Lacl foci to be detected by cell imaging [309]. Second, overexpression of Lacl should be avoided to limit nucleoplasmic Lacl signal which might be confused with DNA bridges
[307]. In fission yeast, the use of Lacl** expressed as a single gene copy from SV40 promoter allowed to avoid chromosome breakage and mitotic LacO-bound Lacl bridges (Figure 19D-F). In all cases, appropriate controls should be employed (i.e. LacO-bound Lacl without an active RFB) to demonstrate that cellular transactions occurring at Lacl foci reflect the activity of the RFB.

The location and distances of $\angle A C O$ arrays relatively to the inducible RFB are one limitation of the system. If possible, both $L a c O$ arrays and the RFB should be replicated by two sister replication forks [330]. Alternatively, LacO arrays can be placed downstream the RFB in a way that the replication fork encounters first the active RFB. The duplication of the $L a C O$ arrays, revealed by two adjacent sister Lacl foci can be used as a readout of the replication of genomic sequences located downstream the RFB, either by the restarted fork or by the opposite fork [333]. In this situation, distances between $\mathrm{LaCO}$ arrays and the RFB should be taken into consideration. Restarted replication forks are often associated with error-prone DNA synthesis whose mutation rate decreases as the restarted fork progresses [336]. Such erroneous DNA synthesis might make the repetitive nature of $\mathrm{LaCO}$-arrays unstable and difficult to maintain over several cell divisions. Increasing the distance between the $\mathrm{LacO}$-arrays and the RFB may render difficult to conclude whether or not a specific DNA repair factor is recruited to the RFB. For example, RPA was reportedly being recruited to the active RFB when Ssb3-mCherry foci were either touching or fully or partially merging with Lacl foci (Figure 19C). Finally, LacO arrays can be located upstream the RFB. However, the processing of replication forks includes the resection of newly replicated strands, up to $3 \mathrm{~Kb}$ in some specific genetics backgrounds, resulting in single stranded $\mathrm{LaCO}$ arrays which will not be bound by Lacl.

\section{A microscopy-based assay to measure DNA double-strand break end resection in single fission yeast cells}

The decision of whether to repair a DNA DSB through canonical (NHEJ or HDR is orchestrated by control over DSB end resection. End resection, or the programmed $3^{\prime}$ to $5^{\prime}$ nucleolytic degradation at the DSB that generates the SSDNA necessary for homology-dependent pairing, commits a DSB to HDR [202, 337, 338]. Thus, repression of resection initiation is essential to promote canonical NHEJ, the preferred repair mechanism in cells prior to DNA replication [337-339]. Numerous factors, such as the Ku heterodimer, the Mre11-Rad50-Nbs1/Xrs2 (MRN/MRX) complex and Sae2/Ctp1/CtIP regulate the initiation of resection $(<100$ bp) [338, 340-344]. Downstream of initiation, long-range resection ( $100 \mathrm{~s}$ bps) can be catalyzed by two machineries: the exonuclease Exo1 and the combination of a RecQ helicase (e.g. Sgs1/Rqh1/BLM) and Dna2 [339, 345-348].

How cells determine and execute the preferential engagement of one of these long-range resection pathways is poorly understood. While Exo1- and Sgs1-depenent end resection appear redundant in budding yeast $[338,348]$, in fission yeast wild type cells utilize specifically the Exo1 pathway [349, 350]. Our work suggests that one consequence of up-regulation of the RecQ helicase-mediated 
A

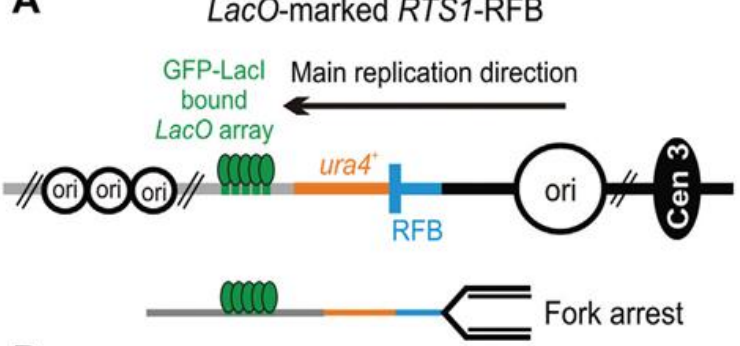

B

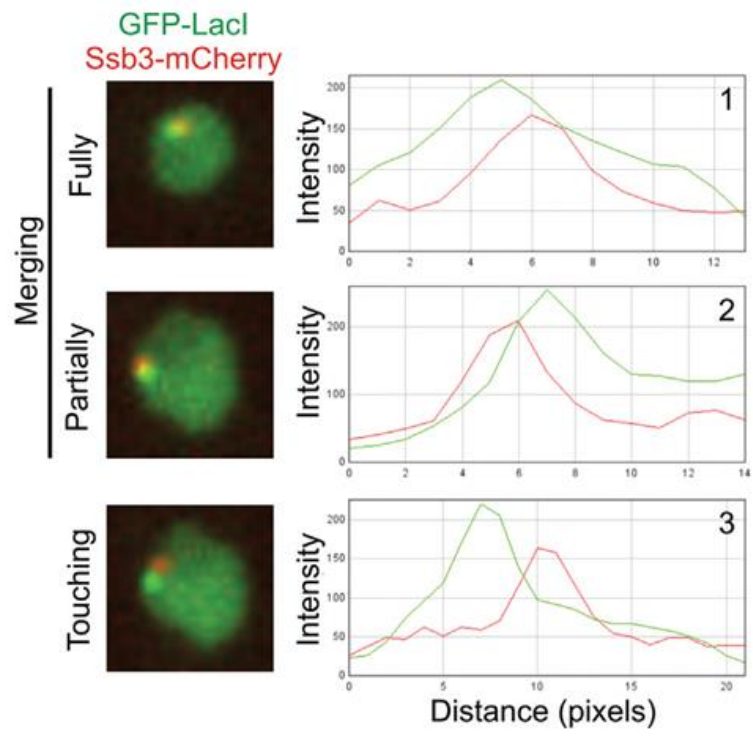

D

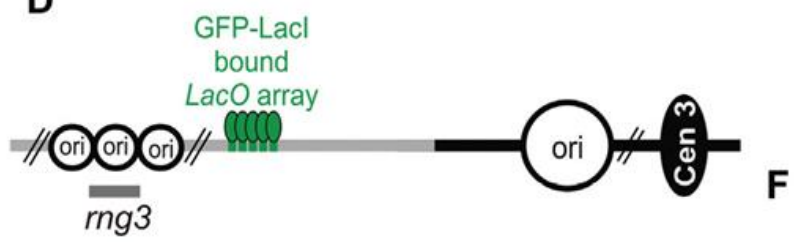

E

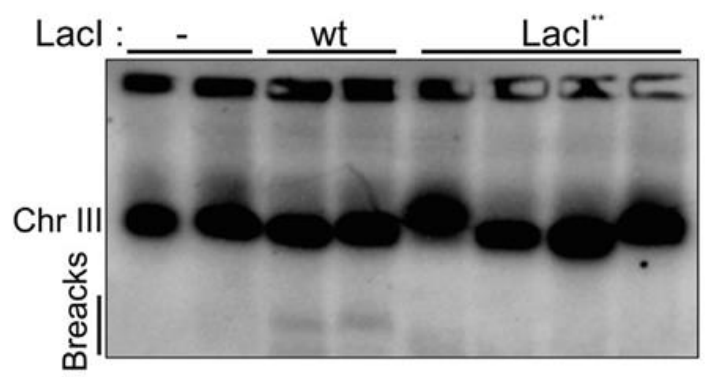

C

$5 \mu \mathrm{m} \quad$ GFP-Lacl

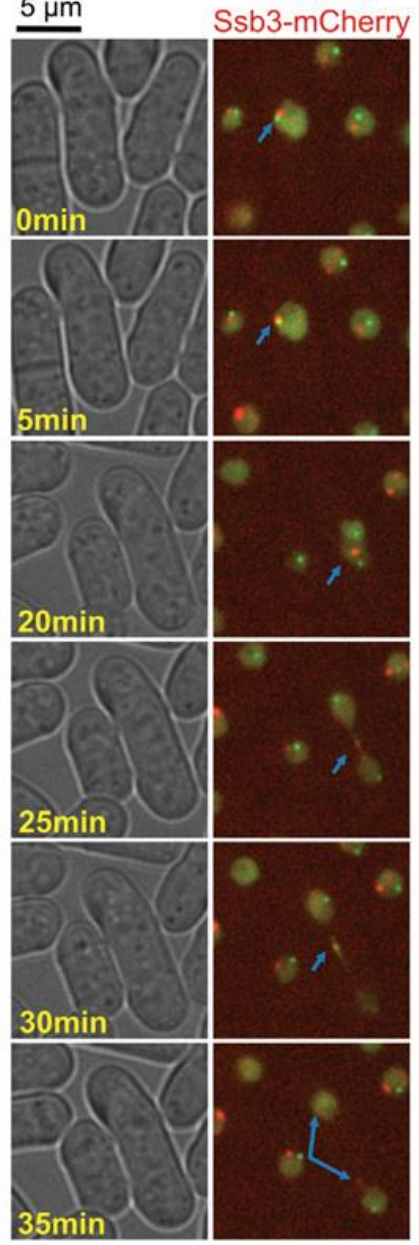

$\mathbf{F}$

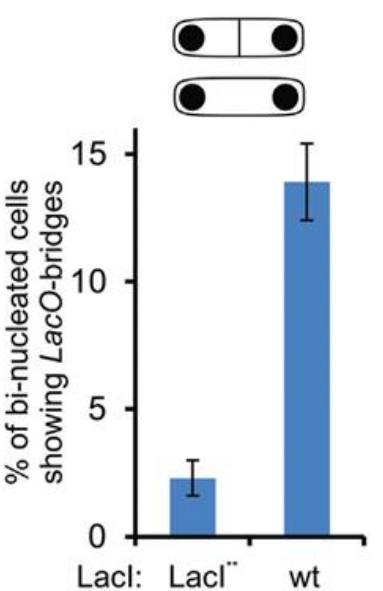

FIGURE 19: A fluorescently-marked RFB to follow in vivo the fate and processing of a single blocked fork in fission yeast. (A) Diagram of the LacO-marked RTS1-RFB locus located on the chromosome III of S. pombe. The blue bar indicates the RTS1-RFB and its polarity. Main replication origins (ori, black circles) located upstream and downstream from the RTS1-RFB are indicated. GFP-Lacl (green ellipses) bound to LacO arrays (green bars) are integrated $\sim 7 \mathrm{~kb}$ away from the RTS1-RFB, on the telomere-proximal side of ura4 gene (red bar). When Rtf1 is expressed, $>90 \%$ of forks emanating from the strong centromere-proximal replication origin, and moving towards the telomere, are blocked. (B) Example of Ssb3mCherry foci being recruited to the active RFB. Three situations were considered: GFP-Lacl and Ssb3-mCherry foci were fully (1) or partially merging (2), or they were touching each other (3). (C) Example of an unprotected fork (identified via the presence of Ssb3-mCherry-positive signal on LacO-marked RTS1-RFB in ON condition, blue arrow) in G2 (time point 0 to 20 minutes) converted into a sister chromatid bridge during mitotic progression (from 20 to 30 minutes) and finally resolved (at 35 minutes). (D) Diagram of the GFP-Lacl bound to LacO arrays located on the chromosome III of S. pombe without RTS1-RFB. The rng3 probe is indicated. (E) Detection of chromosome breakage by Pulse Field Gel electrophoresis followed by Southern-blotting using the rng3 probe, in the following conditions: No Lacl repressor expressed (-), expression of the wild-type (wt) Lacl from a multi-copy expression vector [307], expression of the Lacl ${ }^{* *}$ from a single copy gene and SV40 promoter [333]. (F) Quantification of LacO-bridges in indicated conditions. 
end resection pathway is more rapid (and therefore likely more extensive) resection [350]. However, the mechanisms and consequences of resection mechanism choice remain poorly understood. Further, as chromatin remodeling has been linked to long-range resection efficiency [345] likely many additional factors quantitatively impinge on resection rate, which will require further study. Lastly, as the generation of ssDNA by resection may be uncoupled from loading of repair factors depending on the nuclear context (for example, with heterochromatin [324]), approaches that can monitor DSB end resection within the nuclear context will be enabling technologies of further discovery. Numerous approaches to measure end resection in populations of cells have been developed, such as Southern blotting or quantitative PCR to detect SsDNA generation or protection from restriction enzyme digestion. In intact individual cells, DSB end resection is often inferred by monitoring the loading of ssDNA binding proteins such as RPA or Rad51.

To facilitate the dynamic measurement of resection within the context of the nucleus, we recently developed a live-cell assay capable of revealing long-range resection rates in individual fission yeast cells [350]. This assay is based on signal loss of Lacl-GFP from a LacO array adjacent to a site-specific, inducible DSB. This technology allows the position of a DSB to be interrogated simultaneous with quantitative measures of resection rate with single cell resolution.

\section{Description of the assay}

The principle of the live-cell DSB end resection assay is that the conversion of dsDNA to SSDNA across a LacO array can be monitored by the loss of Lacl-GFP binding. By engineering the LacO array proximal to a site-specific DSB (in this case, a recognition site for the $\mathrm{HO}$ endonuclease from budding yeast, which is absent in fission yeast), processive resection can be monitored (Figure 20A). As the induction of the DSB is neither synchronous nor occurs in all cells, we identify cells that have initiated end resection by the loading of Rad52-mCherry, which colocalizes with the LaclGFP/LacO focus (Figure 20A). While this assay provides indirect information about the kinetics of the initiation of DSB end resection following induction of the $\mathrm{HO}$ nuclease by proxy of the loading of Rad52-mCherry, validation of slow initiation of end resection requires orthogonal approaches to ensure that the efficiency of DSB induction and the loading of Rad52 are unaffected. Thus, this assay is best suited to monitor the kinetics of long-range resection after initiation. Given that we know both that $<300$ bps of resection are required to visualized Rad52-mCherry onto the resulting SSDNA at the DSB $[350,351]$ and the distance (in bps) between the $\mathrm{HO}$ cut site and the start and the end of the LacO array, we can convert the time it takes to resect the full LacO array to a long-range resection rate (Figure 20B). For WT fission yeast cells, which rely on Exo1mediated long-range resection, the median rate is 7.6 $\mathrm{kb} / \mathrm{hr}$ [350].

Further insights into long-range resection mechanism can be obtained by employing genetic perturbations. For example, deletion of the fission yeast RecQ helicase, Rqh1 (orthologous to budding yeast Sgs1 and human BLM), allows Exo1-dependent resection to be studied [350]. While loss of Exo1 disrupts long-range resection in otherwise wild type fission yeast [349], deletion of Crb2 (orthologous to budding yeast Rad9 [352] or human 53BP1 [353-355]) derepresses Rqh1-dependent resection, allowing this mechanism to be explicitly studied [350].

Lastly, because this assay is microscopy-based, it enables the position and dynamics of the DSB to be interrogated simultaneously with end resection. We note that resection from the DSB through the full LacO array ( $13 \mathrm{~kb})$, which takes on average just under 2 hours, appears insufficient to drive targeting to the nuclear periphery. Thus this relocalization of a persistent DSB, described by others in diverse eukaryotes [324, 356-361] and by us in fission yeast [362], appears to require very extensive resection and/or DSB persistence. However, many critical questions into how nuclear subcompartments influence DSB repair mechanism choice and efficiency remain. Future adaptations of this assay will include employing mechanisms to test how tethering the DSB to different nuclear structures and altering the local chromatin state impacts on DSB processing.

\section{Cautionary Notes}

Obtaining reproducible, $\mathrm{HO}$-driven DSB induction in fission yeast

One major challenge is to maintain a strain containing the site-specific nuclease cut site in the presence of the integrated, inducible cognate nuclease, as even transient leakiness of the nuclease promoter leading to nuclease expression can lead to cells with insertion-deletions in the cut site; these cells can then take over the cell population and disrupt the efficiency of inducible DSBs within the observation window. To overcome this, we use the Cre-driven $\mathrm{HO}$ nuclease integration system developed by Tony Carr's laboratory [363], which allows for induction in the presence of uracil. Importantly, we obtain the best results when we transduce the Cre/HO nuclease plasmid just prior to carrying out the assay and induce Cre expression overnight immediately prior to DSB induction by addition of uracil, thereby taking advantage of the high efficiency of the Cre recombinase, which mitigates the need for selection prior to DSB induction. We have also observed that transformants selected for uptake of the Cre donor plasmid demonstrate the most reproducible $\mathrm{HO}$-induced DSB induction if the cells are used between five and ten days after transformation.

\section{Photobleaching}

As this assay interprets the loss of Lacl-GFP intensity, it is essential that the user both mitigates (during the experiment) and assesses (during the analysis) the photostability of the Lacl-GFP signal. Photobleaching is reduced by the addition of the oxygen scavenger, $n$-Propyl gallate (NPG; $0.1 \mathrm{mM}$ ), to both the growth media prior to imaging and the agarose pad on which the cells are maintained and imaged. We suggest initially dissolving the NPG in ethanol at $10 \mathrm{mM}$ followed by a 1:100 dilution in water. Prior to 
A
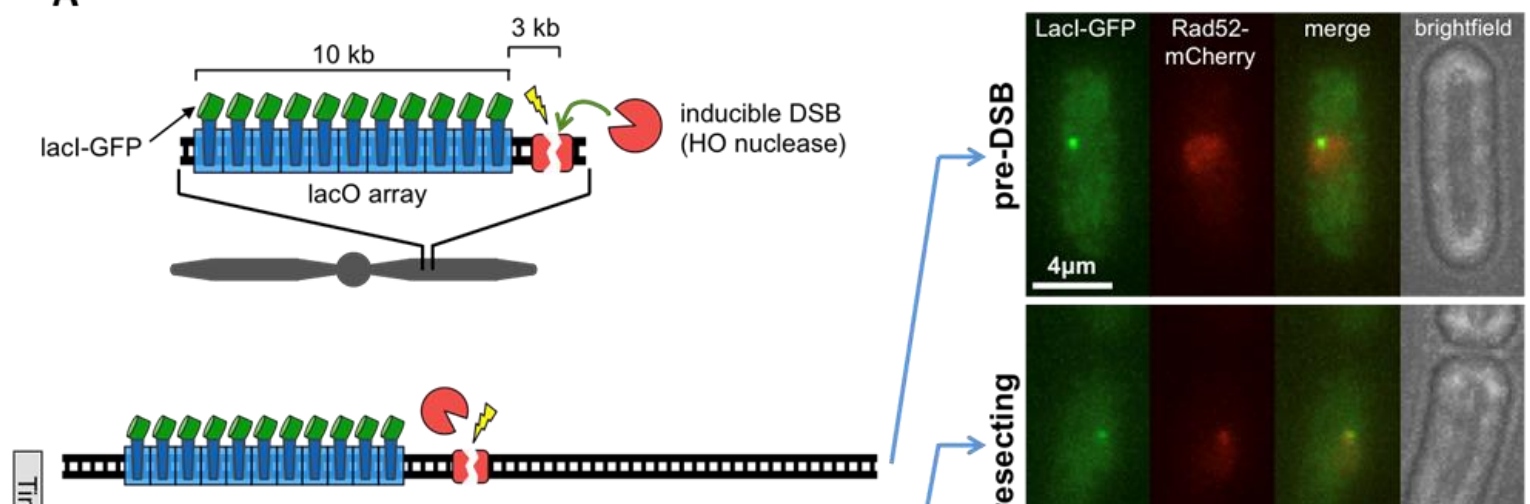

章
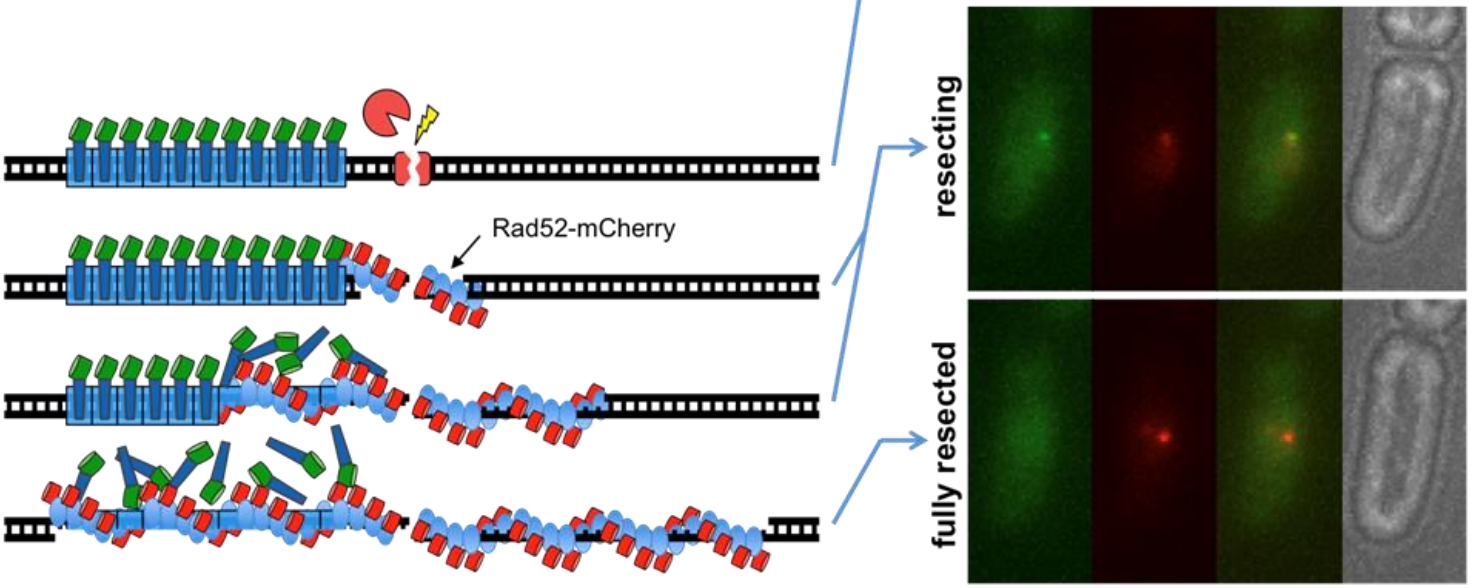

B

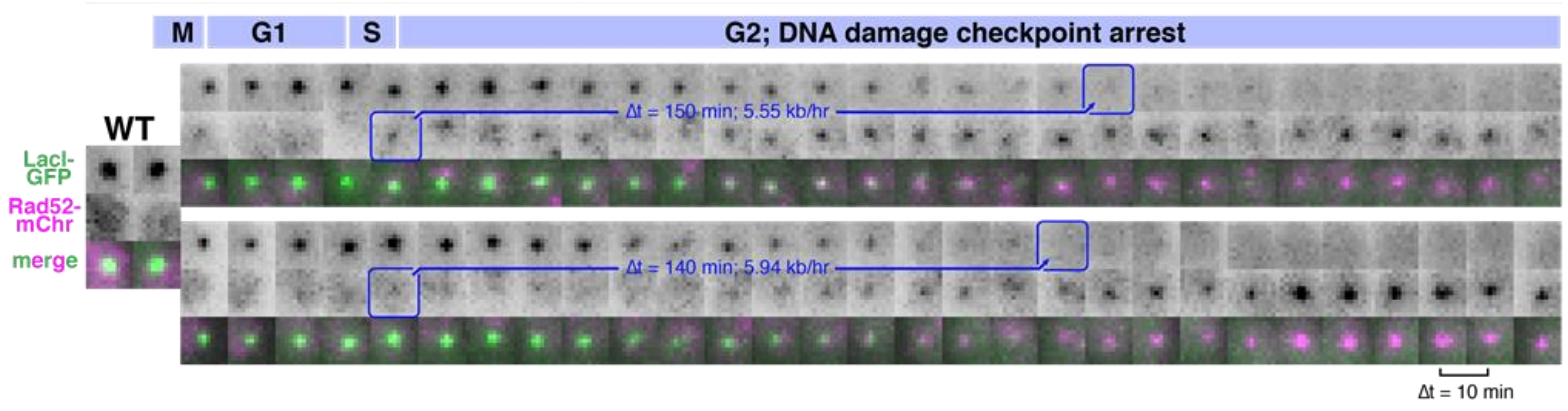

FIGURE 20: Microscopy-based assay for quantitative measurement of DSB end resection rates in single cells. (A) Cartoon diagram of the assay principles, including a recognition site for the $\mathrm{HO}$ nuclease adjacent to a LacO array. Resection drives loss of Lacl-GFP and binding of Rad52-mCherry. On the right are examples of images that highlight the phases of DSB induction and processing. (B) Time-lapse movie of two sister cells that each form a DSB in S phase and undergo resection. The first frame when Rad52-mCherry is detected at the DSB and last frame in which the Lacl-GFP focus is detected are boxed in blue. This gives rise to the resection duration, from which the resection rate can be calculated (blue). The time intervals are 10 minutes.

analysis, each field is assessed for photostability over the $5 \mathrm{~h}$ image acquisition period by monitoring the GFP intensity of control cells at the end of the movie that did not sustain the targeted DSB (that is, lacking a Rad52-mCherry focus). Only fields that maintain robust Lacl-GFP intensity in these control cells are further analyzed.

Validation of DSB induction and resection at the population level by qPCR

Our previous characterization demonstrates that the induction efficiency of the site-specific DSB is largely unaf- fected by mutations that compromise the resection machinery [350]. Moreover, we find that the vast majority of site-specific DSBs commit to homology-directed repair by initiating resection in $\mathrm{G} 2$ fission yeast, which can be assessed by Rad52-mCherry loading at the LacO array [350]. However, confirming the efficiency of DSB induction and the influence on resection at the population level is still recommended. To achieve this, we routinely employ quantitative real-time PCR (qPCR). To determine the DSB induction frequency, PCR primers across the DSB site can be employed; here loss of this product compared to a control 
PCR product can be quantified [350]. It should be noted, however, that since this is a signal-loss experiment, the PCR product across the cut site typically decreases by only $\sim 15-20 \%$. An orthogonal method to investigate resection at the population level is to monitor protection from restriction enzyme digestion by qPCR [204, 349] using the same assay strains and conditions used for microscopy. Importantly, in this case the efficiency of resection can only be timed relative to the induction of the $\mathrm{HO}$ nuclease (addition of uracil to the media), and thus includes the delay for $\mathrm{HO}$ nuclease production, individual cells to reach $S$ phase (when the HO nuclease has access to cut its sitespecific recognition site), and the process of resection itself Moreover, if there is a delay in resection initiation this will dominate the observations, making it impossible to independently measure the long-range resection rate. In the microscopy-based assay we isolate the long-range resection phase, which can be observed even if relatively fewer cells initiate timely resection (that is, load Rad52-mCherry at the LacO array).

\section{An irreparable DSB}

One additional caveat is that resection monitored using this assay is extensive (over $10 \mathrm{~kb}$ ) driven by the fact that this is an irreparable DSB. We have evidence that in the vast majority of cells both sister chromatids are cut by the HO nuclease (data not shown). When the assay is carried out in haploid cells (as described), this renders this a lethal DSB that is subjected to prolonged resection.

\section{Conclusion}

Microscopy-based approaches that enable real-time visualization of distinct events in DSB processing and repair hold great promise for providing quantitative, dynamic, singlecell information previously inaccessible with genetic marker assays. Combined with assays of repair outcome, we are at the cusp of realizing new insights into the mechanisms that underlie the observed inhomogeneity across the genome in the susceptibility to DNA damage and/or the outcome of DNA repair $[45,364]$. Combining the power of yeast genetics with existing and future live-cell DSB repair assays will continue to provide powerful insights into the cell biology of DNA repair.

\section{SUMMARY}

Here we have presented an overview of three broad areas of assays for mutagenesis and recombination in bacterial and fungal systems, focusing on genetic, molecular and cytological approaches. Each singular assay is described with its utility and caveats, and has been shown to advance the field and our knowledge regarding the mechanisms and central protein components of the multiple DNA repair and recombination pathways. The synergistic application of multiple assays that includes assays from each broad category has made the bacterial and fungal systems the foundation for recombination and repair studies.

\section{ACKNOWLEDGMENTS}

HLK was supported by NIH grant R01-CA146940. AA was supported by the European research Council (ERC2014ADG669898 TARLOOP), the Spanish Ministry of Economy and Competitiveness (BFU2016-75058-P), and the Junta de Andalucía (BIO1238). JLA was supported by NIH grant R35G-GM119788. CHF was supported by NIH grants P01GM105473 and R01-GM122880. SMG was supported by the Swiss national Science Foundation and the Novartis Research Foundation. DAG was supported by National Institutes of Health (Intramural Research Program Project Z1AES103266. JEH was supported by NIH grants R35GM127029 and P01-GM105473. GI was supported by NIH grants R01-GM125650 and R01-GM080600. SJ-R was supported by NIH grant R35-GM118077. MCK was supported by National Science Foundation Graduate Research Fellowship DGE-1122492, NIH training grant T32-GM007223 and NIH grant DP20D008429. RDK and CDP were supported by NIH grants R01-GM26017 and R01-GM50006. AK was supported by NIH grant R01-GM073115. SAEL was supported by grants from Agence Nationale de la Recherche ANR-14CE10-0010-01 and the Foundation pour la Recherche Médicale Equipe FRM DEQ20160334889. SEL was supported by NIH grant R01-GM071011. SMM was supported by NIH grants R01-GM60987 and P01-GM105473. TDP was supported by NIH grant R35-GM118020. SMR was supported by a gift from WM Keck Foundation, NIH Director's Pioneer Award DP1-CA174424 and NIH grant R35-GM122598. DMF was supported by the Cancer Prevention and Research Institute of Texas, Baylor College of Medicine Comprehensive Cancer Training Program Postdoctoral Fellowship RP160283. RR was supported by NIH grant R35-GM118180 and $\mathrm{JM}-\mathrm{H}$ was supported by a Marie Curie International Outgoing Fellowship and the ANR-12-PDOC-0035-01. LSS was supported by NIH grant R35-GM126997. PZ was supported by National Science Centre Poland 2015/19/103859 and FNP First TEAM/2016-1/9. NK was supported by NIH grant R01-GM116007 and the Welch Foundation (AU1875). ML was supported by grants from the Danish Agency for Science, Technology and Innovation, the Villum Foundation and the Danish National Research Foundation (DNRF115). AM was supported by NIH grants R35-GM127006 and R03ES029306.

\section{SUPPLEMENTAL MATERIAL}

All supplemental data for this article are available online at www.microbialcell.com.

\section{CONFLICT OF INTEREST}

The authors declare no conflict of interest.

\section{COPYRIGHT}

(C) 2019 Klein et al. This is an open-access article released under the terms of the Creative Commons Attribution (CC BY) license, which allows the unrestricted use, distribution, and reproduction in any medium, provided the original author and source are acknowledged. 
Please cite this article as: Hannah L. Klein, et al. (2019). Guidelines for DNA recombination and repair studies: Cellular assays of DNA repair pathways. Microbial Cell 6(1): 1-64. doi: 10.15698/mic2019.01.664

\section{REFERENCES}

1. Haracska L, Unk I, Johnson RE, Johansson E, Burgers PM, Prakash S, Prakash $L$ (2001). Roles of yeast DNA polymerases delta and zeta and of Rev1 in the bypass of abasic sites. Genes Dev 15(8): 945-954. doi: 10.1101/gad.882301

2. Lippert MJ, Kim N, Cho JE, Larson RP, Schoenly NE, O'Shea SH, JinksRobertson $S$ (2011). Role for topoisomerase 1 in transcriptionassociated mutagenesis in yeast. Proc Natl Acad Sci U S A 108(2): 698703. doi: 10.1073/pnas.1012363108

3. Pursell ZF, Isoz I, Lundstrom EB, Johansson E, Kunkel TA (2007). Yeast DNA polymerase epsilon participates in leading-strand DNA replication. Science 317(5834): 127-130. doi: 10.1126/science.1144067

4. Nick McElhinny SA, Gordenin DA, Stith CM, Burgers PM, Kunkel TA (2008). Division of labor at the eukaryotic replication fork. Mol Cell 30(2): 137-144. doi: 10.1016/j.molcel.2008.02.022

5. Kim N, Mudrak SV, Jinks-Robertson S (2011). The dCMP transferase activity of yeast Rev1 is biologically relevant during the bypass of endogenously generated AP sites. DNA Repair 10(12): 1262-1271. doi: 10.1016/j.dnarep.2011.09.017

6. Williams TM, Fabbri RM, Reeves JW, Crouse GF (2005). A new reversion assay for measuring all possible base pair substitutions in Saccharomyces cerevisiae. Genetics 170(3): 1423-1426. doi: 10.1534/genetics.105.042697

7. Zaret KS, Sherman $F$ (1985). $\alpha$-Aminoadipate as a primary nitrogen source for Saccharomyces cerevisiae mutants. J Bacteriol 162(2): 579583. PMID: 3921525

8. Greene CN, Jinks-Robertson S (1997). Frameshift intermediates in homopolymer runs are removed efficiently by yeast mismatch repair proteins. Mol Cell Biol 17: 2844-2850. doi: 10.1128/mcb.17.5.2844

9. Harfe BD, Jinks-Robertson S (1999). Removal of frameshift intermediates by mismatch repair proteins in Saccharomyces cerevisiae. Mol Cell Biol 19: 4766-4773. doi: 10.1128/mcb.19.7.4766

10. Harfe BD, Jinks-Robertson S (2000). Sequence composition and context effects on the generation and repair of frameshift intermediates in mononucleotide runs in Saccharomyces cerevisiae. Genetics 156: 571-578. PMID: 11014807

11. Gragg H, Harfe BD, Jinks-Robertson S (2002). Base composition of mononucleotide runs affects DNA polymerase slippage and removal of frameshift intermediates by mismatch repair in Saccharomyces cerevisiae. Mol Cell Biol 22(24): 8756-8762. doi: 10.1128/mcb.22.24.8756-8762.2002

12. Minesinger BK, Jinks-Robertson S (2005). Roles of RAD6 epistasis group members in spontaneous Polל-dependent translesion synthesis in Saccharomyces cerevisiae. Genetics 169: 1939-1955. doi: 10.1534/genetics.104.033894

13. Harfe BD, Jinks-Robertson S (2000). DNA polymerase $\zeta$ introduces multiple mutations when bypassing spontaneous DNA damage in Saccharomyces cerevisiae. Mol Cell 6: 1491-1499. doi: 10.1016/s10972765(00)00145-3

14. Lehner K, Mudrak SV, Minesinger BK, Jinks-Robertson S (2012). Frameshift mutagenesis: the roles of primer-template misalignment and the nonhomologous end-joining pathway in Saccharomyces cerevisiae. Genetics 190(2): 501-510. doi: 10.1534/genetics.111.134890
15. Kim N, Abdulovic AL, Gealy R, Lippert MJ, Jinks-Robertson S (2007) Transcription-associated mutagenesis in yeast is directly proportional to the level of gene expression and influenced by the direction of DNA replication. DNA Repair 6(9): 1285-1296. doi:

\subsection{6/j.dnarep.2007.02.023}

16. Kim N, Cho JE, Li YC, Jinks-Robertson S (2013). RNA:DNA hybrids initiate quasi-palindrome-associated mutations in highly transcribed yeast DNA. PLoS genetics 9(11): e1003924. doi 10.1371/journal.pgen.1003924

17. Cho JE, Kim N, Li YC, Jinks-Robertson S (2013). Two distinct mechanisms of Topoisomerase 1-dependent mutagenesis in yeast. DNA Repair 12(3): 205-211. doi: 10.1016/j.dnarep.2012.12.004

18. Guo X, Hum YF, Lehner K, Jinks-Robertson S (2017). Regulation of hetDNA length during mitotic double-strand break repair in yeast. Mol Cell 67(4): 539-549. doi: 10.1016/j.molcel.2017.07.009

19. Pardo B, Gomez-Gonzalez B, Aguilera A (2009). DNA repair in mammalian cells: DNA double-strand break repair: how to fix a broken relationship. Cell Mol Life Sci 66(6): 1039-1056. doi: 10.1007/s00018009-8740-3

20. San Filippo J, Sung P, Klein H (2008). Mechanism of eukaryotic homologous recombination. Annu Rev Biochem 77(229-257. doi: 10.1146/annurev.biochem.77.061306.125255

21. Kadyk LC, Hartwell LH (1992). Sister chromatids are preferred over homologs as substrates for recombinational repair in Saccharomyces cerevisiae. Genetics 132(2): 387-402. PMID: 1427035

22. Johnson RD, Jasin M (2000). Sister chromatid gene conversion is a prominent double-strand break repair pathway in mammalian cells. EMBO J 19(13): 3398-3407. doi: 10.1093/emboj/19.13.3398

23. Gonzalez-Barrera S, Cortes-Ledesma F, Wellinger RE, Aguilera A (2003). Equal sister chromatid exchange is a major mechanism of double-strand break repair in yeast. Mol Cell 11(6): 1661-1671. doi: 10.1016/s1097-2765(03)00183-7

24. Klein HL, Petes TD (1981). Intrachromosomal gene conversion in yeast. Nature 289(5794): 144-148. doi: 10.1038/289144a0

25. Jackson JA, Fink GR (1981). Gene conversion between duplicated genetic elements in yeast. Nature 292(5821): 306-311. doi: $10.1038 / 292306 a 0$

26. Klein $\mathrm{HL}$ (1984). Lack of association between intrachromosomal gene conversion and reciprocal exchange. Nature 310(5980): 748-753. doi: $10.1038 / 310748 \mathrm{a} 0$

27. Cortes-Ledesma F, Prado F, Aguilera A (2007). Sister-chromatid recombination. Molecular Genetics of Recombination 17:221-241. doi: 10.1007/4735_2006_0213

28. Heyer WD, Ehmsen KT, Liu J (2010). Regulation of homologous recombination in eukaryotes. Annu Rev Genet 44: 113-139. doi: 10.1146/annurev-genet-051710-150955

29. Gomez-Gonzalez B, Ruiz JF, Aguilera A (2011). Genetic and molecular analysis of mitotic recombination in Saccharomyces cerevisiae. Methods Mol Biol 745: 151-172. doi: 10.1007/978-161779-129-1_10

30. Fasullo MT, Davis RW (1987). Recombinational substrates designed to study recombination between unique and repetitive 
sequences in vivo. Proc Natl Acad Sci U S A 84(17): 6215-6219. doi: 10.1073/pnas.84.17.6215

31. Cortes-Ledesma F, Aguilera A (2006). Double-strand breaks arising by replication through a nick are repaired by cohesin-dependent sister-chromatid exchange. EMBO Rep 7(9): 919-926. doi: 10.1038/sj.embor.7400774

32. Munoz-Galvan S, Jimeno S, Rothstein R, Aguilera A (2013). Histone H3K56 acetylation, Rad52, and non-DNA repair factors control doublestrand break repair choice with the sister chromatid. PLoS Genet 9(1): e1003237. doi: 10.1371/journal.pgen.1003237

33. Mascioli DW, Haber JE (1980). A CIS-Acting Mutation within the MATa Locus of SACCHAROMYCES CEREVISIAE That Prevents Efficient Homothallic Mating-Type Switching. Genetics 94(2): 341-360. PMID: 17249002

34. Cortes-Ledesma F, Tous C, Aguilera A (2007). Different genetic requirements for repair of replication-born double-strand breaks by sister-chromatid recombination and break-induced replication. Nucleic Acids Res 35(19): 6560-6570. doi: 10.1093/nar/gkm488

35. Munoz-Galvan $S$, Garcia-Rubio M, Ortega $P$, Ruiz JF, Jimeno $S$, Pardo B, Gomez-Gonzalez B, Aguilera A (2017). A new role for Rrm3 in repair of replication-born DNA breakage by sister chromatid recombination. PLoS Genet 13(5): e1006781. doi: 10.1371/journal.pgen.1006781

36. Munoz-Galvan S, Tous C, Blanco MG, Schwartz EK, Ehmsen KT, West SC, Heyer WD, Aguilera A (2012). Distinct roles of Mus81, Yen1, SIx1-SIx4, and Rad1 nucleases in the repair of replication-born doublestrand breaks by sister chromatid exchange. Mol Cell Biol 32(9): 1592 1603. doi: 10.1128/MCB.00111-12

37. Munoz-Galvan S, Lopez-Saavedra A, Jackson SP, Huertas $P$, CortesLedesma F, Aguilera A (2013). Competing roles of DNA end resection and non-homologous end joining functions in the repair of replicationborn double-strand breaks by sister-chromatid recombination. Nucleic Acids Res 41(3): 1669-1683. doi: 10.1093/nar/gks1274

38. De Piccoli G, Cortes-Ledesma F, Ira G, Torres-Rosell J, Uhle S, Farmer S, Hwang JY, Machin F, Ceschia A, McAleenan A, CordonPreciado $V$, Clemente-Blanco A, Vilella-Mitjana $F$, Ullal $P$, Jarmuz $A$, Leitao B, Bressan D, Dotiwala F, Papusha A, Zhao X, Myung K, Haber JE, Aguilera A, Aragon L (2006). Smc5-Smc6 mediate DNA doublestrand-break repair by promoting sister-chromatid recombination. Nat Cell Biol 8(9): 1032-1034. doi: 10.1038/ncb1466

39. Aguilera A, Klein HL (1988). Genetic control of intrachromosoma recombination in Saccharomyces cerevisiae. I. Isolation and genetic characterization of hyper-recombination mutations. Genetics 119(4): 779-790. PMID: 3044923

40. Wallis JW, Chrebet G, Brodsky G, Rolfe M, Rothstein R (1989). A hyper-recombination mutation in $S$. cerevisiae identifies a novel eukaryotic topoisomerase. Cell 58(2): 409-419. doi: 10.1016/0092 8674(89)90855-6

41. Smirnova M, Van Komen S, Sung P, Klein HL (2004). Effects of tumor-associated mutations on Rad54 functions. J Biol Chem 279(23): 24081-24088. doi: 10.1074/jbc.M402719200

42. Spell RM, Jinks-Robertson S (2004). Determination of mitotic recombinaiton rates by fluctuation analysis in Saccharomyces cerevisiae. Methods Mol Biol 262(3-12. doi: 10.1385/1-59259-7610:003

43. Lea DE, Coulson CA (1949). The distribution of the numbers of mutants in bacterial populations. J Genet 49(3): 264-285. doi: $10.1007 /$ bf02986080

44. Luria SE, Delbruck M (1943). Mutations of Bacteria from Virus Sensitivity to Virus Resistance. Genetics 28(6): 491-511. PMID: 17247100
45. Lang GI, Murray AW (2008). Estimating the per-base-pair mutation rate in the yeast Saccharomyces cerevisiae. Genetics 178(1): 67-82. doi: 10.1534/genetics.107.071506

46. Wu X, Strome ED, Meng Q, Hastings PJ, Plon SE, Kimmel M (2009) A robust estimator of mutation rates. Mutat Res 661(1-2): 101-109. doi: 10.1016/j.mrfmmm.2008.11.015

47. Merker RJ, Klein HL (2002). hpr1Delta affects ribosomal DNA recombination and cell life span in Saccharomyces cerevisiae. Mol Cell Biol 22(2): 421-429. doi: 10.1128/mcb.22.2.421-429.2002

48. Klein HL (1988). Different types of recombination events are controlled by the RAD1 and RAD52 genes of Saccharomyces cerevisiae. Genetics 120(2): 367-377. PMID: 3058548

49. Ho CK, Mazon G, Lam AF, Symington LS (2010). Mus81 and Yen1 promote reciprocal exchange during mitotic recombination to maintain genome integrity in budding yeast. Mol Cell 40(6): 988-1000. doi: 10.1016/j.molcel.2010.11.016

50. Huang KN, Symington LS (1994). Mutation of the gene encoding protein kinase $\mathrm{C} 1$ stimulates mitotic recombination in Saccharomyces cerevisiae. Mol Cell Biol 14(9): 6039-6045. doi: 10.1128/mcb.14.9.6039

51. Mozlin AM, Fung CW, Symington LS (2008). Role of the Saccharomyces cerevisiae Rad51 paralogs in sister chromatid recombination. Genetics 178(1): 113-126. doi: 10.1534/genetics.107.082677

52. Rattray AJ, Symington LS (1994). Use of a chromosomal inverted repeat to demonstrate that the RAD51 and RAD52 genes of Saccharomyces cerevisiae have different roles in mitotic recombination. Genetics 138(3): 587-595. PMID: 7851757

53. Bai Y, Davis AP, Symington LS (1999). A novel allele of RAD52 that causes severe DNA repair and recombination deficiencies only in the absence of RAD51 or RAD59. Genetics 153(3): 1117-1130. PMID: 10545446

54. Bai Y, Symington LS (1996). A Rad52 homolog is required for RAD51-independent mitotic recombination in Saccharomyces cerevisiae. Genes Dev 10(16): 2025-2037. doi: 10.1101/gad.10.16.2025

55. Chen W, Jinks-Robertson S (1998). Mismatch repair proteins regulate heteroduplex formation during mitotic recombination in yeast. Mol Cell Biol 18(11): 6525-6537. doi: 10.1128/mcb.18.11.6525

56. Mott C, Symington LS (2011). RAD51-independent inverted-repeat recombination by a strand-annealing mechanism. DNA Repair 10(4): 408-415. doi: 10.1016/j.dnarep.2011.01.007

57. Fung CW, Mozlin AM, Symington LS (2009). Suppression of the double-strand-break-repair defect of the Saccharomyces cerevisiae rad57 mutant. Genetics 181(4): 1195-1206. doi: 10.1534/genetics.109.100842

58. Petes TD, Malone RE, Symington LS (1991). Recombination in Yeast. In: The Molecular and Cellular Biology of the Yeast Saccharomyces: Genome Dynamics, Protein Synthesis, and Energetics. Cold Spring Harbor Laboratory Press; pp. 407 - 521.

59. Barbera MA, Petes TD (2006). Selection and analysis of spontaneous reciprocal mitotic cross-overs in Saccharomyces cerevisiae. Proc Natl Acad Sci U S A 103(34): 12819-12824. doi: 10.1073/pnas.0605778103

60. Chua $P$, Jinks-Robertson S (1991). Segregation of recombinant chromatids following mitotic crossing over in yeast. Genetics $129(2)$ : 359-369. PMID: 1660426

61. Lee PS, Greenwell PW, Dominska M, Gawel M, Hamilton M, Petes TD (2009). A fine-structure map of spontaneous mitotic crossovers in 
the yeast Saccharomyces cerevisiae. PLoS Genet 5(3): e1000410. doi: 10.1371/journal.pgen.1000410

62. St Charles J, Hazkani-Covo E, Yin Y, Andersen SL, Dietrich FS, Greenwell PW, Malc E, Mieczkowski P, Petes TD (2012). Highresolution genome-wide analysis of irradiated (UV and gamma-rays) diploid yeast cells reveals a high frequency of genomic loss of heterozygosity (LOH) events. Genetics 190(4): 1267-1284. doi: 10.1534/genetics.111.137927

63. St Charles J, Petes TD (2013). High-resolution mapping of spontaneous mitotic recombination hotspots on the $1.1 \mathrm{Mb}$ arm of yeast chromosome IV. PLoS Genet 9(4): e1003434. doi: 10.1371/journal.pgen.1003434

64. Tang W, Dominska M, Greenwell PW, Harvanek Z, Lobachev KS, Kim HM, Narayanan V, Mirkin SM, Petes TD (2011). Friedreich's ataxia $(\mathrm{GAA}) \mathrm{n}^{*}(\mathrm{TTC}) \mathrm{n}$ repeats strongly stimulate mitotic crossovers in Saccharomyces cerevisae. PLoS Genet 7(1): e1001270. doi: 10.1371/journal.pgen.1001270

65. Yin Y, Petes TD (2013). Genome-wide high-resolution mapping of UV-induced mitotic recombination events in Saccharomyces cerevisiae. PLoS Genet 9(10): e1003894. doi: 10.1371/journal.pgen.1003894

66. Yin $Y$, Petes TD (2015). Recombination between Homologous Chromosomes Induced by Unrepaired UV-Generated DNA Damage Requires Mus81p and Is Suppressed by Mms2p. PLoS Genet 11(3): e1005026. doi: 10.1371/journal.pgen.1005026

67. Andersen SL, Sloan RS, Petes TD, Jinks-Robertson S (2015). Genome-destabilizing effects associated with top1 loss or accumulation of top1 cleavage complexes in yeast. PLoS Genet 11(4): e1005098. doi: 10.1371/journal.pgen.1005098

68. Song W, Dominska M, Greenwell PW, Petes TD (2014). Genomewide high-resolution mapping of chromosome fragile sites in Saccharomyces cerevisiae. Proc Natl Acad Sci U S A. doi: 10.1073/pnas.1406847111

69. Zheng DQ, Zhang K, Wu XC, Mieczkowski PA, Petes TD (2016) Global analysis of genomic instability caused by DNA replication stress in Saccharomyces cerevisiae. Proc Natl Acad Sci U S A 113(50): E8114E8121. doi: 10.1073/pnas.1618129113

70. Putnam CD, Kolodner RD (2017). Pathways and Mechanisms that Prevent Genome Instability in Saccharomyces cerevisiae. Genetics 206(3): 1187-1225. doi: 10.1534/genetics.112.145805

71. Lupski JR (2015). Structural variation mutagenesis of the human genome: Impact on disease and evolution. Environ Mol Mutagen 56(5): 419-436. doi: 10.1002/em.21943

72. Campbell PJ, Yachida S, Mudie LJ, Stephens PJ, Pleasance ED, Stebbings LA, Morsberger LA, Latimer C, McLaren S, Lin ML, McBride DJ, Varela I, Nik-Zainal SA, Leroy C, Jia M, Menzies A, Butler AP, Teague JW, Griffin CA, Burton J, Swerdlow H, Quail MA, Stratton MR, lacobuzio-Donahue C, Futreal PA (2010). The patterns and dynamics of genomic instability in metastatic pancreatic cancer. Nature 467(7319): 1109-1113. doi: 10.1038/nature09460

73. Gundem G, Van Loo P, Kremeyer B, Alexandrov LB, Tubio JM, Papaemmanuil E, Brewer DS, Kallio HM, Hognas G, Annala M, Kivinummi K, Goody V, Latimer C, O'Meara S, Dawson KJ, Isaacs W, Emmert-Buck MR, Nykter M, Foster C, Kote-Jarai Z, Easton D, Whitaker HC, Group IPU, Neal DE, Cooper CS, Eeles RA, Visakorpi T, Campbell PJ, McDermott U, Wedge DC, et al. (2015). The evolutionary history of lethal metastatic prostate cancer. Nature 520(7547): 353 357. doi: 10.1038/nature14347

74. Mitelman F, Johansson B, Mertens F (2017). Mitelman database of chromosome aberrations and gene fusions in cancer. Available at:
https://cgap.nci.nih.gov/Chromosomes/Mitelman

[accessed 20.03.2018].

75. Friedberg EC, Walker GC, Siede W, Wood RD, Schultz RA Ellenberger T (2006). DNA Repair and Mutagenesis. ASM Press, Washington DC; doi: 10.1128/9781555816704

76. Putnam CD, Srivatsan A, Nene RV, Martinez SL, Clotfelter SP, Bell SN, Somach SB, de Souza JES, Fonseca AF, de Souza SJ, Kolodner RD (2016). A genetic network that suppresses genome rearrangements in Saccharomyces cerevisiae and contains defects in cancers. Nat Commun 7: 11256. doi: 10.1038/ncomms11256

77. Chen C, Kolodner RD (1999). Gross chromosomal rearrangement in Saccharomyces cerevisiae replication and recombination defective mutants. Nat Genet 23(1): 81-85. doi: 10.1038/12687

78. Putnam CD, Hayes TK, Kolodner RD (2009). Specific pathways prevent duplication-mediated genome rearrangements. Nature 460(7258): 984-989. doi: 10.1038/nature08217

79. Srivatsan A, Putnam CD, Kolodner RD (2018). Analyzing Genome Rearrangements in Saccharomyces cerevisiae. Methods Mol Biol 1672: 43-61. doi: 10.1007/978-1-4939-7306-4_5

80. Pennaneach V, Kolodner RD (2009). Stabilization of dicentric translocations through secondary rearrangements mediated by multiple mechanisms in S. cerevisiae. PLoS One 4(7): e6389. doi: 10.1371/journal.pone.0006389

81. Nene RV, Putnam CD, Li BZ, Nguyen KG, Srivatsan A, Campbell CS Desai A, Kolodner RD (2018). Cdc73 suppresses genome instability by mediating telomere homeostasis. PLoS Genet 14(1): e1007170. doi: 10.1371/journal.pgen.1007170

82. Liang J, Li BZ, Tan AP, Kolodner RD, Putnam CD, Zhou H (2018). SUMO E3 ligase Mms21 prevents spontaneous DNA damage induced genome rearrangements. PLoS Genet 14(3): e1007250. doi: 10.1371/journal.pgen.1007250

83. Budworth H, McMurray CT (2013). A brief history of triplet repeat diseases. Methods Mol Biol 1010: 3-17. doi: 10.1007/978-1-62703411-1_1

84. Den Dunnen WFA (2017). Trinucleotide repeat disorders. Handb Clin Neurol 145: 383-391. doi: 10.1016/B978-0-12-802395-2.00027-4

85. Lee DY, McMurray CT (2014). Trinucleotide expansion in disease: why is there a length threshold? Curr Opin Genet Dev 26: 131-140. doi: $10.1016 /$ j.gde.2014.07.003

86. Richards RI, Sutherland GR (1992). Dynamic mutations: a new class of mutations causing human disease. Cell 70(5): 709-712. doi: 10.1016/0092-8674(92)90302-s

87. Neil AJ, Kim JC, Mirkin SM (2017). Precarious maintenance of simple DNA repeats in eukaryotes. Bioessays 39(9). doi: 10.1002/bies.201700077

88. Zhao XN, Usdin K (2015). The Repeat Expansion Diseases: The dark side of DNA repair. DNA Repair 32: 96-105. doi: 10.1016/j.dnarep.2015.04.019

89. Polleys EJ, House NCM, Freudenreich CH (2017). Role of recombination and replication fork restart in repeat instability. DNA Repair 56: 156-165. doi: 10.1016/j.dnarep.2017.06.018

90. Budworth H, McMurray CT (2013). Bidirectional transcription of trinucleotide repeats: roles for excision repair. DNA Repair 12(8): 672 684. doi: 10.1016/j.dnarep.2013.04.019

91. Lin $Y$, Hubert L, Jr., Wilson JH (2009). Transcription destabilizes triplet repeats. Mol Carcinog 48(4): 350-361. doi: 10.1002/mc.20488

92. Shishkin AA, Voineagu I, Matera R, Cherng N, Chernet BT, Krasilnikova MM, Narayanan V, Lobachev KS, Mirkin SM (2009). Large- 
scale expansions of Friedreich's ataxia GAA repeats in yeast. Mol Cell 35(1): 82-92. doi: 10.1016/j.molcel.2009.06.017

93. Shah KA, McGinty RJ, Egorova VI, Mirkin SM (2014). Coupling transcriptional state to large-scale repeat expansions in yeast. Cell Rep 9(5): 1594-1602. doi: 10.1016/j.celrep.2014.10.048

94. Yu X, Gabriel A (1999). Patching broken chromosomes with extranuclear cellular DNA. Mol Cell 4(5): 873-881. doi: 10.1016/s10972765(00)80397-4

95. Shah KA, Shishkin AA, Voineagu I, Pavlov YI, Shcherbakova PV Mirkin SM (2012). Role of DNA polymerases in repeat-mediated genome instability. Cell Rep 2(5): 1088-1095. doi: 10.1016/j.celrep.2012.10.006

96. McGinty RJ, Rubinstein RG, Neil AJ, Dominska M, Kiktev D, Petes TD, Mirkin SM (2017). Nanopore sequencing of complex genomic rearrangements in yeast reveals mechanisms of repeat-mediated double-strand break repair. Genome Res 27(12): 2072-2082. doi: 10.1101/gr.228148.117

97. Moore A, Dominska M, Greenwell P, Aksenova AY, Mirkin S, Petes $\mathrm{T}$ (2018). Genetic Control of Genomic Alterations Induced in Yeast by Interstitial Telomeric Sequences. Genetics 209(2):425-438. doi: 10.1534/genetics.118.300950

98. Aksenova AY, Greenwell PW, Dominska M, Shishkin AA, Kim JC, Petes TD, Mirkin SM (2013). Genome rearrangements caused by interstitial telomeric sequences in yeast. Proc Natl Acad Sci U S A 110(49): 19866-19871. doi: 10.1073/pnas.1319313110

99. Radchenko EA, McGinty RJ, Aksenova AY, Neil AJ, Mirkin SM (2018). Quantitative Analysis of the Rates for Repeat-Mediated Genome Instability in a Yeast Experimental System. Methods Mol Biol 1672(421-438. doi: 10.1007/978-1-4939-7306-4 29

100. Neil AJ, Liang MU, Khristich AN, Shah KA, Mirkin SM (2018). RNADNA hybrids promote the expansion of Friedreich's ataxia (GAA)n repeats via break-induced replication. Nucleic Acids Res 46(7):3487 3497 doi: 10.1093/nar/gky099

101. Cherng N, Shishkin AA, Schlager LI, Tuck RH, Sloan L, Matera R, Sarkar PS, Ashizawa T, Freudenreich CH, Mirkin SM (2011). Expansions, contractions, and fragility of the spinocerebellar ataxia type 10 pentanucleotide repeat in yeast. Proc Natl Acad Sci U S A 108(7): 2843-2848. doi: 10.1073/pnas.1009409108

102. Aksenova AY, Han G, Shishkin AA, Volkov KV, Mirkin SM (2015) Expansion of Interstitial Telomeric Sequences in Yeast. Cell Rep 13(8): 1545-1551. doi: 10.1016/j.celrep.2015.10.023

103. McGinty RJ, Puleo F, Aksenova AY, Hisey JA, Shishkin AA, Pearson EL, Wang ET, Housman DE, Moore C, Mirkin SM (2017). A Defective mRNA Cleavage and Polyadenylation Complex Facilitates Expansions of Transcribed (GAA)n Repeats Associated with Friedreich's Ataxia. Cell Rep 20(10): 2490-2500. doi: 10.1016/j.celrep.2017.08.051

104. Usdin K, House NC, Freudenreich CH (2015). Repeat instability during DNA repair: Insights from model systems. Crit Rev Biochem Mol Biol 50(2): 142-167. doi: 10.3109/10409238.2014.999192

105. Schulz VP, Zakian VA (1994). The saccharomyces PIF1 DNA helicase inhibits telomere elongation and de novo telomere formation. Cell 76(1): 145-155. doi: 10.1016/0092-8674(94)90179-1

106. Callahan JL, Andrews KJ, Zakian VA, Freudenreich CH (2003). Mutations in yeast replication proteins that increase CAG/CTG expansions also increase repeat fragility. Mol Cell Biol 23(21): 78497860. doi: 10.1128/mcb.23.21.7849-7860.2003

107. Kerrest A, Anand RP, Sundararajan R, Bermejo R, Liberi G, Dujon $B$, Freudenreich $\mathrm{CH}$, Richard GF (2009). SRS2 and SGS1 prevent chromosomal breaks and stabilize triplet repeats by restraining recombination. Nat Struct Mol Biol 16(2): 159-167. doi: 10.1038/nsmb.1544

108. Zhang $\mathrm{H}$, Freudenreich $\mathrm{CH}$ (2007). An AT-rich sequence in human common fragile site FRA16D causes fork stalling and chromosome breakage in S. cerevisiae. Mol Cell 27(3): 367-379. doi: 10.1016/j.molcel.2007.06.012

109. Lu S, Wang G, Bacolla A, Zhao J, Spitser S, Vasquez KM (2015). Short Inverted Repeats Are Hotspots for Genetic Instability: Relevance to Cancer Genomes. Cell Rep S2211-1247(15): 00197-7. doi: 10.1016/j.celrep.2015.02.039

110. Zhao J, Wang G, Del Mundo IM, McKinney JA, Lu X, Bacolla A, Boulware SB, Zhang $\mathrm{C}$, Zhang $\mathrm{H}$, Ren $\mathrm{P}$, Freudenreich $\mathrm{CH}$, Vasquez $\mathrm{KM}$ (2018). Distinct Mechanisms of Nuclease-Directed DNA-StructureInduced Genetic Instability in Cancer Genomes. Cell Rep 22(5): 12001210. doi: 10.1016/j.celrep.2018.01.014

111. Freudenreich $\mathrm{CH}$, Stavenhagen JB, Zakian VA (1997). Stability of a CTG/CAG trinucleotide repeat in yeast is dependent on its orientation in the genome. Mol Cell Biol 17(4): 2090-2098. doi: 10.1128/mcb.17.4.2090

112. Su XA, Freudenreich $\mathrm{CH}$ (2017). Cytosine deamination and base excision repair cause $\mathrm{R}$-loop-induced CAG repeat fragility and instability in Saccharomyces cerevisiae. Proc Natl Acad Sci U S A 114(40): E8392-E8401. doi: 10.1073/pnas.1711283114

113. Koch MR, House NCM, Cosetta CM, Jong RM, Salomon CG, Joyce $\mathrm{CE}$, Philips EA, Su XA, Freudenreich $\mathrm{CH}$ (2018). The Chromatin Remodeler Isw1 Prevents CAG Repeat Expansions During Transcription in Saccharomyces cerevisiae. Genetics 208(3): 963-976. doi: 10.1534/genetics.117.300529

114. Foster PL (2006). Methods for determining spontaneous mutation rates. Methods Enzymol 409: 195-213. doi: 10.1016/S0076 6879(05)09012-9

115. Polleys EJ, Freudenreich $\mathrm{CH}$ (2018). Methods to Study Repeat Fragility and Instability in Saccharomyces cerevisiae. Methods Mol Biol 1672: 403-419. doi: 10.1007/978-1-4939-7306-4_28

116. Pluta AF, Zakian VA (1989). Recombination occurs during telomere formation in yeast. Nature 337(6206): 429-433. doi: $10.1038 / 337429 \mathrm{a0}$

117. Sundararajan R, Gellon L, Zunder RM, Freudenreich CH (2010). Double-strand break repair pathways protect against CAG/CTG repeat expansions, contractions and repeat-mediated chromosomal fragility in Saccharomyces cerevisiae. Genetics 184(1): 65-77. doi: 10.1534/genetics.109.111039

118. Sebat J, Lakshmi B, Troge J, Alexander J, Young J, Lundin P, Mane $\mathrm{S}$, Massa $\mathrm{H}$, Walker $\mathrm{M}$, Chi M, Navin N, Lucito R, Healy J, Hicks J, Ye K, Reiner A, Gilliam TC, Trask B, Patterson N, Zetterberg A, Wigler M (2004). Large-scale copy number polymorphism in the human genome. Science 305(5683): 525-528. doi: 10.1126/science.1098918

119. lafrate AJ, Feuk L, Rivera MN, Listewnik ML, Donahoe PK, Qi Y, Scherer SW, Lee C (2004). Detection of large-scale variation in the human genome. Nat Genet 36(9): 949-951. doi: 10.1038/ng1416

120. Lupski JR (2007). Genomic rearrangements and sporadic disease. Nat Genet 39(7 Suppl): S43-47. doi: 10.1038/ng2084

121. Conover HN, Argueso JL (2016). Contrasting mechanisms of de novo copy number mutagenesis suggest the existence of different classes of environmental copy number mutagens. Environ Mol Mutagen 57(1): 3-9. doi: 10.1002/em.21967

122. Narayanan V, Mieczkowski PA, Kim HM, Petes TD, Lobachev KS (2006). The pattern of gene amplification is determined by the chromosomal location of hairpin-capped breaks. Cell 125(7): 12831296. doi: 10.1016/j.cell.2006.04.042 
123. Zhang $H$, Zeidler AF, Song W, Puccia CM, Malc E, Greenwell PW, Mieczkowski PA, Petes TD, Argueso JL (2013). Gene copy-number variation in haploid and diploid strains of the yeast Saccharomyces cerevisiae. Genetics 193(3): 785-801. doi 10.1534/genetics.112.146522

124. Robberecht C, Voet T, Esteki MZ, Nowakowska BA, Vermeesch JR (2013). Nonallelic homologous recombination between retrotransposable elements is a driver of de novo unbalanced translocations. Genome Res 23(3): 411-418. doi 10.1101/gr.145631.112

125. Campbell IM, Gambin T, Dittwald $P$, Beck $C R$, Shuvarikov $A$ Hixson P, Patel A, Gambin A, Shaw CA, Rosenfeld JA, Stankiewicz P (2014). Human endogenous retroviral elements promote genome instability via non-allelic homologous recombination. BMC biology 12 74. doi: 10.1186/s12915-014-0074-4

126. Startek M, Szafranski $P$, Gambin T, Campbell IM, Hixson $P$, Shaw CA, Stankiewicz P, Gambin A (2015). Genome-wide analyses of LINELINE-mediated nonallelic homologous recombination. Nucleic Acids Res 43(4): 2188-2198. doi: 10.1093/nar/gku1394

127. Hastings PJ, Ira G, Lupski JR (2009). A microhomology-mediated break-induced replication model for the origin of human copy number variation. PLoS genetics 5(1): e1000327. doi 10.1371/journal.pgen.1000327

128. Putnam CD, Srivatsan A, Nene RV, Martinez SL, Clotfelter SP, Bell SN, Somach SB, de Souza JE, Fonseca AF, de Souza SJ, Kolodner RD (2016). A genetic network that suppresses genome rearrangements in Saccharomyces cerevisiae and contains defects in cancers. Nat Commun 7: 11256. doi: 10.1038/ncomms11256

129. Holmes A, Haber JE (1999). Physical monitoring of HO-induced homologous recombination. Methods Mol Biol 113: 403-415. doi: 10.1385/1-59259-675-4:403

130. Sugawara N, Haber JE (2012). Monitoring DNA recombination initiated by $\mathrm{HO}$ endonuclease. Methods Mol Biol 920: 349-370. doi: 10.1007/978-1-61779-998-3_25

131. Paques F, Haber JE (1999). Multiple pathways of recombination induced by double-strand breaks in Saccharomyces cerevisiae. Microbiol Mol Biol Rev 63(2): 349-404. PMID: 10357855

132. Hariharan PV, Hutchinson F (1973). Neutral sucrose gradient sedimentation of very large DNA from Bacillus subtilis. II. Doublestrand breaks formed by gamma ray irradiation of the cells. J Mol Biol 75(3): 479-494. doi: 10.1016/0022-2836(73)90455-5

133. Debenham PG, Webb MB (1988). The isolation and preliminary characterisation of a novel Escherichia coli mutant rorB with enhanced sensitivity to ionising radiation. Mol Gen Genet 215(1): 161164. doi: 10.1007/bf00331319

134. Singh NP, Stephens RE, Singh H, Lai H (1999). Visual quantification of DNA double-strand breaks in bacteria. Mutat Res 429(2): 159-168. PMID: 10526201

135. Fernandez JL, Cartelle M, Muriel L, Santiso R, Tamayo M, Goyanes V, Gosalvez J, Bou G (2008). DNA fragmentation in microorganisms assessed in situ. Appl Environ Microbiol 74(19): 5925-5933. doi: 10.1128/AEM.00318-08

136. Johnston RF, Pickett SC, Barker DL (1990). Autoradiography using storage phosphor technology. Electrophoresis 11(5): 355-360. doi: 10.1002/elps.1150110503

137. Upham LV, Englert DF (2003). Radionuclide imaging. In L'Annunziata MF

Handbook of radioactivity analysis. Academic Press, San Diego; pp. 647-692.
138. Allardet-Servent A, Michaux-Charachon S, Jumas-Bilak E, Karayan $L$, Ramuz M (1993). Presence of one linear and one circular chromosome in the Agrobacterium tumefaciens C58 genome. J Bacteriol 175(24): 7869-7874. doi: 10.1128/jb.175.24.7869-7874.1993

139. Belfaiza J, Postic D, Bellenger E, Baranton G, Girons IS (1993). Genomic fingerprinting of Borrelia burgdorferi sensu lato by pulsedfield gel electrophoresis. J Clin Microbiol 31(11): 2873-2877. PMID: 8263170

140. Fan JB, Rochet M, Gaillardin C, Smith CL (1992). Detection and characterization of a ring chromosome in the fission yeast Schizosaccharomyces pombe. Nucleic Acids Res 20(22): 5943-5945. PMID: 1461727

141. Mendez-Alvarez S, Pavon V, Esteve I, Guerrero R, Gaju N (1995). Analysis of bacterial genomes by pulsed field gel electrophoresis. Microbiologia 11(3): 323-336. PMID: 7576348

142. Smith CL, Econome JG, Schutt A, KIco S, Cantor CR (1987). A physical map of the Escherichia coli K12 genome. Science 236(4807): 1448-1453. doi: 10.1126/science.3296194

143. Van der Ploeg LH, Smits M, Ponnudurai T, Vermeulen A Meuwissen JH, Langsley G (1985). Chromosome-sized DNA molecules of Plasmodium falciparum. Science 229(4714): 658-661. doi: 10.1126/science.3895435

144. Brosch R, Chen J, Luchansky JB (1994). Pulsed-field fingerprinting of listeriae: identification of genomic divisions for Listeria monocytogenes and their correlation with serovar. Appl Environ Microbiol 60(7): 2584-2592. PMID: 8074531

145. Kristjansson M, Samore MH, Gerding DN, DeGirolami PC, Bettin KM, Karchmer AW, Arbeit RD (1994). Comparison of restriction endonuclease analysis, ribotyping, and pulsed-field gel electrophoresis for molecular differentiation of Clostridium difficile strains. J Clin Microbiol 32(8): 1963-1969. PMID: 7989550

146. Zhang Y, Mazurek GH, Cave MD, Eisenach KD, Pang Y, Murphy DT, Wallace RJ, Jr. (1992). DNA polymorphisms in strains of Mycobacterium tuberculosis analyzed by pulsed-field gel electrophoresis: a tool for epidemiology. J Clin Microbiol 30(6): 15511556. PMID: 1352518

147. Orbach MJ, Vollrath D, Davis RW, Yanofsky C (1988). An electrophoretic karyotype of Neurospora crassa. Mol Cell Biol 8(4): 1469-1473. doi: 10.1128/mcb.8.4.1469

148. Schwartz DC, Cantor CR (1984). Separation of yeast chromosomesized DNAs by pulsed field gradient gel electrophoresis. Cell 37(1): 67 75. doi: 10.1016/0092-8674(84)90301-5

149. Goering RV (2010). Pulsed field gel electrophoresis: a review of application and interpretation in the molecular epidemiology of infectious disease. Infect Genet Evol 10(7): 866-875. doi: 10.1016/j.meegid.2010.07.023

150. Struelens M, De Ryck R, Deplano A (2001). Analysis of microbial genomic macro-restriction patterns by pulsed-field gel electrophoresis (PFGE) typing. In: New Approaches for the Generation and Analysis of Microbial Typing Data. Elsevier, Amsterdam.

151. Fuke M, Thomas CA, Jr. (1970). Isolation of open-circular DNA molecules by retention in agar gels. J Mol Biol 52(2): 395-397. doi: 10.1016/0022-2836(70)90040-9

152. Mickel S, Arena V, Jr., Bauer W (1977). Physical properties and gel electrophoresis behavior of R12-derived plasmid DNAs. Nucleic Acids Rese 4(5): 1465-1482. doi: 10.1093/nar/4.5.1465

153. Schindler CW, Krolewski JJ, Rush MG (1982). Selective trapping of circular double-stranded DNA molecules in solidifying agarose. Plasmid 7(3): 263-270. doi: 10.1016/0147-619x(82)90007-5 
154. Serwer P, Watson RH, Hayes SJ (1987). Multidimensional analysis of intracellular bacteriophage T7 DNA: effects of amber mutations in genes 3 and 19. J Virol 61(11): 3499-3509. PMID: 2822958

155. Beverley SM (1988). Characterization of the 'unusual' mobility of large circular DNAs in pulsed field-gradient electrophoresis. Nucleic Acids Res 16(3): 925-939. doi: 10.1093/nar/16.3.925

156. Game JC, Sitney KC, Cook VE, Mortimer RK (1989). Use of a ring chromosome and pulsed-field gels to study interhomolog recombination, double-strand DNA breaks and sister-chromatid exchange in yeast. Genetics 123(4): 695-713. PMID: 2693206

157. Michel B, Ehrlich SD, Uzest M (1997). DNA double-strand breaks caused by replication arrest. EMBO J 16(2): 430-438. doi: 10.1093/emboj/16.2.430

158. Westmoreland J, Ma W, Yan Y, Van Hulle K, Malkova A, Resnick MA (2009). RAD50 is required for efficient initiation of resection and recombinational repair at random, gamma-induced double-strand break ends. PLoS Genet 5(9): e1000656. doi 10.1371/journal.pgen.1000656

159. Budke B, Kuzminov A (2010). Production of clastogenic DNA precursors by the nucleotide metabolism in Escherichia coli. Mol Microbiol 75(1): 230-245. doi: 10.1111/j.1365-2958.2009.06994.x

160. Kouzminova EA, Rotman E, Macomber L, Zhang J, Kuzminov A (2004). RecA-dependent mutants in Escherichia coli reveal strategies to avoid chromosomal fragmentation. Proc Natl Acad Sci U S A 101(46): 16262-16267. doi: 10.1073/pnas.0405943101

161. Kouzminova EA, Kuzminov A (2006). Fragmentation of replicating chromosomes triggered by uracil in DNA. J Mol Biol 355(1): 20-33. doi: 10.1016/j.jmb.2005.10.044

162. Kouzminova EA, Kuzminov A (2008). Patterns of chromosomal fragmentation due to uracil-DNA incorporation reveal a novel mechanism of replication-dependent double-stranded breaks. Mol Microbiol 68(1): 202-215. doi: 10.1111/j.1365-2958.2008.06149.x

163. Kouzminova EA, Kuzminov A (2012). Chromosome demise in the wake of ligase-deficient replication. Mol Microbiol 84(6): 1079-1096. doi: 10.1111/j.1365-2958.2012.08076.x

164. Kouzminova EA, Kadyrov FF, Kuzminov A (2017). RNase HII Saves rnhA Mutant Escherichia coli from R-Loop-Associated Chromosomal Fragmentation. J Mol Biol 429(19): 2873-2894. doi: 10.1016/j.jmb.2017.08.004

165. Rotman E, Khan SR, Kouzminova E, Kuzminov A (2014) Replication fork inhibition in seqA mutants of Escherichia coli triggers replication fork breakage. Mol Microbiol 93(1): 50-64. doi: 10.1111/mmi.12638

166. Khan SR, Kuzminov A (2012). Replication forks stalled at ultraviolet lesions are rescued via RecA and RuvABC protein-catalyzed disintegration in Escherichia coli. J Biol Chem 287(9): 6250-6265. doi: 10.1074/jbc.M111.322990

167. Khan SR, Kuzminov A (2013). Trapping and breaking of in vivo nicked DNA during pulsed field gel electrophoresis. Anal Biochem 443(2): 269-281. doi: 10.1016/j.ab.2013.06.001

168. Khan SR, Kuzminov A (2017). Pulsed-field gel electrophoresis does not break $E$. coli chromosome undergoing excision repair after UV irradiation. Anal Biochem 526: 66-68. doi: 10.1016/j.ab.2017.03.019

169. Mahaseth T, Kuzminov A (2015). Cyanide enhances hydrogen peroxide toxicity by recruiting endogenous iron to trigger catastrophic chromosomal fragmentation. Mol Microbiol 96(2): 349-367. doi: 10.1111/mmi.12938

170. Mahaseth T, Kuzminov A (2016). Prompt repair of hydrogen peroxide-induced DNA lesions prevents catastrophic chromosomal fragmentation. DNA Repair 41: 42-53. doi: 10.1016/j.dnarep.2016.03.012

171. Fangman WL (1978). Separation of very large DNA molecules by gel electrophoresis. Nucleic Acids Res 5(3): 653-665. doi: 10.1093/nar/5.3.653

172. Serwer $P$ (1981). Improvements in procedures for electrophoresis in dilute agarose gels. Anal Biochem 112(2): 351-356. doi 10.1016/0003-2697(81)90304-3

173. Rudolph CJ, Upton AL, Lloyd RG (2009). Replication fork collisions cause pathological chromosomal amplification in cells lacking RecG DNA translocase. Mol Microbiol 74(4): 940-955. doi: 10.1111/j.13652958.2009.06909.x

174. Romling U, Tummler B (2000). Achieving 100\% typeability of Pseudomonas aeruginosa by pulsed-field gel electrophoresis. J Clin Microbiol 38(1): 464-465. PMID: 10618146

175. Khan SR, Kuzminov A (2017). Degradation of RNA during lysis of Escherichia coli cells in agarose plugs breaks the chromosome. PLoS One 12(12): e0190177. doi: 10.1371/journal.pone.0190177

176. Sandell LL, Zakian VA (1993). Loss of a yeast telomere: arrest, recovery, and chromosome loss. Cell 75(4): 729-739. doi: 10.1016/0092-8674(93)90493-a

177. Lee CS, Haber JE (2015). Mating-type Gene Switching in Saccharomyces cerevisiae. Microbiol Spectr 3(2): MDNA3-0013-2014. doi: 10.1128/microbiolspec.MDNA3-0013-2014

178. Moore JK, Haber JE (1996). Cell cycle and genetic requirements of two pathways of nonhomologous end-joining repair of double-strand breaks in Saccharomyces cerevisiae. Mol Cell Biol 16(5): 2164-2173. doi: $10.1128 / \mathrm{mcb} \cdot 16.5 .2164$

179. Connolly $\mathrm{B}$, White $\mathrm{Cl}$, Haber JE (1988). Physical monitoring of mating type switching in Saccharomyces cerevisiae. Mol Cell Biol 8(6): 2342-2349. doi: 10.1128/mcb.8.6.2342

180. Rudin N, Haber JE (1988). Efficient repair of HO-induced chromosomal breaks in Saccharomyces cerevisiae by recombination between flanking homologous sequences. Mol Cell Biol 8(9): 3918 3928. doi: 10.1128/mcb.8.9.3918

181. Lydeard JR, Jain S, Yamaguchi M, Haber JE (2007). Break-induced replication and telomerase-independent telomere maintenance require Pol32. Nature 448(7155): 820-823. doi: 10.1038/nature06047

182. Plessis A, Perrin A, Haber JE, Dujon B (1992). Site-specific recombination determined by I-Scel, a mitochondrial group I intronencoded endonuclease expressed in the yeast nucleus. Genetics 130(3): 451-460. PMID: 1551570

183. White $\mathrm{Cl}$, Haber JE (1990). Intermediates of recombination during mating type switching in Saccharomyces cerevisiae. EMBO J 9(3): 663 673. doi: 10.1002/j.1460-2075.1990.tb08158.x

184. Sugawara N, Wang X, Haber JE (2003). In vivo roles of Rad52, Rad54, and Rad55 proteins in Rad51-mediated recombination. Mol Cell 12(1): 209-219. doi: 10.1016/s1097-2765(03)00269-7

185. Haber JE (2016). A Life Investigating Pathways That Repair Broken Chromosomes. Annu Rev Genet 50: 1-28. doi: 10.1146/annurevgenet-120215-035043

186. Anand R, Beach A, Li K, Haber J (2017). Rad51-mediated double strand break repair and mismatch correction of divergent substrates. Nature 544(7650): 377-380. doi: 10.1038/nature22046

187. Lee CS, Wang RW, Chang HH, Capurso D, Segal MR, Haber JE (2016). Chromosome position determines the success of doublestrand break repair. Proc Natl Acad Sci U S A 113(2): E146-154. doi: 10.1073/pnas.1523660113 
188. Coic E, Martin J, Ryu T, Tay SY, Kondev J, Haber JE (2011). Dynamics of homology searching during gene conversion in Saccharomyces cerevisiae revealed by donor competition. Genetics 189(4): 1225-1233. doi: 10.1534/genetics.111.132738

189. Mehta A, Beach A, Haber JE (2017). Homology Requirements and Competition between Gene Conversion and Break-Induced Replication during Double-Strand Break Repair. Mol Cell 65(3): 515526 e513. doi: 10.1016/j.molcel.2016.12.003

190. Jain S, Sugawara N, Mehta A, Ryu T, Haber JE (2016). Sgs1 and Mph1 Helicases Enforce the Recombination Execution Checkpoint During DNA Double-Strand Break Repair in Saccharomyces cerevisiae. Genetics 203(2): 667-675. doi: 10.1534/genetics.115.184317

191. Hicks WM, Kim M, Haber JE (2010). Increased mutagenesis and unique mutation signature associated with mitotic gene conversion. Science 329(5987): 82-85. doi: 10.1126/science.1191125

192. Tsaponina O, Haber JE (2014). Frequent Interchromosomal Template Switches during Gene Conversion in S. cerevisiae. Mol Cell 55(4): 615-625. doi: 10.1016/j.molcel.2014.06.025

193. Anand RP, Tsaponina O, Greenwell PW, Lee CS, Du W, Petes TD, Haber JE (2014). Chromosome rearrangements via template switching between diverged repeated sequences. Genes Dev 28(21): 2394-2406. doi: 10.1101/gad.250258.114

194. Haber JE (2002). Uses and abuses of HO endonuclease. Methods Enzymol 350: 141-164. doi: 10.1016/s0076-6879(02)50961-7

195. Watson AT, Werler P, Carr AM (2011). Regulation of gene expression at the fission yeast Schizosaccharomyces pombe urg1 locus. Gene 484(1-2): 75-85. doi: 10.1016/j.gene.2011.05.028

196. Sunder S, Greeson-Lott NT, Runge KW, Sanders SL (2012). A new method to efficiently induce a site-specific double-strand break in the fission yeast Schizosaccharomyces pombe. Yeast 29(7): 275-291. doi 10.1002/yea.2908

197. Ohle C, Tesorero R, Schermann G, Dobrev N, Sinning I, Fischer T (2016). Transient RNA-DNA Hybrids Are Required for Efficient DoubleStrand Break Repair. Cell 167(4): 1001-1013 e1007. doi: 10.1016/j.cell.2016.10.001

198. Muscarella DE, Vogt VM (1993). A mobile group I intron from Physarum polycephalum can insert itself and induce point mutations in the nuclear ribosomal DNA of Saccharomyces cerevisiae. Mol Cell Biol 13(2): 1023-1033. doi: 10.1128/mcb.13.2.1023

199. Wang J, Eisenstatt JR, Audryc J, Corneliusd K, Shaughnessyc M, Berknerc KL, Runge KW (2017). A heterochromatin domain forms gradually at a new telomere and is highly dynamic at stable telomeres. Mol Cell Biol 38(15): e00393-17. doi: 10.1128/MCB.00393-17

200. Matsuyama A, Shirai A, Yashiroda Y, Kamata A, Horinouchi S, Yoshida M (2004). pDUAL, a multipurpose, multicopy vector capable of chromosomal integration in fission yeast. Yeast 21(15): 1289-1305. doi: 10.1002/yea.1181

201. Blaikley EJ, Tinline-Purvis H, Kasparek TR, Marguerat S, Sarkar S, Hulme L, Hussey S, Wee BY, Deegan RS, Walker CA, Pai CC, Bahler J, Nakagawa T, Humphrey TC (2014). The DNA damage checkpoint pathway promotes extensive resection and nucleotide synthesis to facilitate homologous recombination repair and genome stability in fission yeast. Nucleic Acids Res 42(9): 5644-5656. doi: 10.1093/nar/gku190

202. Symington LS, Gautier J (2011). Double-strand break end resection and repair pathway choice. Annu Rev Genet 45: 247-271. doi: 10.1146/annurev-genet-110410-132435

203. Booth C, Griffith E, Brady G, Lydall D (2001). Quantitative amplification of single-stranded DNA (QAOS) demonstrates that cdc13-1 mutants generate ssDNA in a telomere to centromere direction. Nucleic Acids Research 29(21): 4414-4422. doi: 10.1093/nar/29.21.4414

204. Zierhut C, Diffley JF (2008). Break dosage, cell cycle stage and DNA replication influence DNA double strand break response. Embo J 27(13): 1875-1885. doi: 10.1038/emboj.2008.111

205. Che J, Smith S, Kim YJ, Shim EY, Myung K, Lee SE (2015). HyperAcetylation of Histone H3K56 Limits Break-Induced Replication by Inhibiting Extensive Repair Synthesis. PLoS Genet 11(2): e1004990. doi: 10.1371/journal.pgen.1004990

206. Mimitou EP, Symington LS (2009). DNA end resection: many nucleases make light work. DNA Repair 8(9): 983-995. doi: 10.1016/j.dnarep.2009.04.017

207. Marians KJ (2018). Lesion Bypass and the Reactivation of Stalled Replication Forks. Annu Rev Biochem 87:217-238. doi: 10.1146/annurev-biochem-062917-011921

208. Broomfield S, Hryciw T, Xiao W (2001). DNA postreplication repair and mutagenesis in Saccharomyces cerevisiae. Mutat Res 486(3): 167-184. doi: 10.1016/s0921-8777(01)00091-x

209. Branzei D (2011). Ubiquitin family modifications and template switching. FEBS Lett 585(18): 2810-2817. doi: 10.1016/j.febslet.2011.04.053

210. Mullen JR, Kaliraman V, Ibrahim SS, Brill SJ (2001). Requirement for three novel protein complexes in the absence of the Sgs1 DNA helicase in Saccharomyces cerevisiae. Genetics 157(1): 103-118. PMID: 11139495

211. Klein HL (2001). Mutations in recombinational repair and in checkpoint control genes suppress the lethal combination of srs2Delta with other DNA repair genes in Saccharomyces cerevisiae. Genetics 157(2): 557-565. PMID: 11156978

212. Gangloff S, Soustelle C, Fabre F (2000). Homologous recombination is responsible for cell death in the absence of the Sgs1 and Srs2 helicases. Nat Genet 25(2): 192-194. doi: 10.1038/76055

213. Keyamura K, Arai K, Hishida T (2016). Srs2 and Mus81-Mms4 Prevent Accumulation of Toxic Inter-Homolog Recombination Intermediates. PLoS Genet 12(7): e1006136. doi: 10.1371/journal.pgen.1006136

214. Elango R, Sheng Z, Jackson J, DeCata J, Ibrahim Y, Pham NT, Liang DH, Sakofsky CJ, Vindigni A, Lobachev KS, Ira G, Malkova A (2017). Break-induced replication promotes formation of lethal joint molecules dissolved by Srs2. Nat Commun 8(1): 1790 . doi: 10.1038/s41467-017-01987-2

215. Chung WH, Zhu Z, Papusha A, Malkova A, Ira G (2010). Defective resection at DNA double-strand breaks leads to de novo telomere formation and enhances gene targeting. PLoS Genet 6(5): e1000948. doi: 10.1371/journal.pgen.1000948

216. Deem A, Barker K, Vanhulle K, Downing B, Vayl A, Malkova A (2008). Defective break-induced replication leads to half-crossovers in Saccharomyces cerevisiae. Genetics 179(4): 1845-1860. doi: 10.1534/genetics.108.087940

217. Malkova A, Naylor ML, Yamaguchi M, Ira G, Haber JE (2005). RAD51-dependent break-induced replication differs in kinetics and checkpoint responses from RAD51-mediated gene conversion. Mol Cell Biol 25(3): 933-944. doi: 10.1128/MCB.25.3.933-944.2005

218. Saini N, Ramakrishnan S, Elango R, Ayyar S, Zhang Y, Deem A, Ira G, Haber JE, Lobachev KS, Malkova A (2013). Migrating bubble during break-induced replication drives conservative DNA synthesis. Nature 502(7471): 389-392. doi: 10.1038/nature12584

219. Ruff P, Donnianni RA, Glancy E, Oh J, Symington LS (2016). RPA Stabilization of Single-Stranded DNA Is Critical for Break-Induced 
Replication. Cell Rep 17(12): 3359-3368. doi: 10.1016/j.celrep.2016.12.003

220. Rohner S, Gasser SM, Meister P (2008). Modules for cloning-free chromatin tagging in Saccharomyces cerevisae. Yeast 25(3): 235-239. doi: 10.1002/yea.1580

221. Burch LH, Yang Y, Sterling JF, Roberts SA, Chao FG, Xu H, Zhang L, Walsh J, Resnick MA, Mieczkowski PA, Gordenin DA (2011). Damageinduced localized hypermutability. Cell Cycle 10(7): 1073-1085. doi: 10.4161/cc.10.7.15319

222. Chan K, Sterling JF, Roberts SA, Bhagwat AS, Resnick MA, Gordenin DA (2012). Base damage within single-strand DNA underlies in vivo hypermutability induced by a ubiquitous environmental agent. PLoS Genet 8(12): e1003149. doi: 10.1371/journal.pgen.1003149

223. Roberts SA, Sterling J, Thompson C, Harris S, Mav D, Shah R, Klimczak L, Kryukov GV, Malc E, Mieczkowski PA, Resnick MA, Gordenin DA (2012). Clustered mutations in yeast and in human cancers can arise from damaged long single-strand DNA regions. Mol Cell 46(4): 424-435. doi: 10.1016/j.molcel.2012.03.030

224. Sakofsky CJ, Roberts SA, Malc E, Mieczkowski PA, Resnick MA, Gordenin DA, Malkova A (2014). Break-induced replication is a source of mutation clusters underlying kataegis. Cell Rep 7(5): 1640-1648. doi: 10.1016/j.celrep.2014.04.053

225. Yang Y, Sterling J, Storici F, Resnick MA, Gordenin DA (2008). Hypermutability of damaged single-strand DNA formed at doublestrand breaks and uncapped telomeres in yeast Saccharomyces cerevisiae. PLoS Genet 4(11): e1000264. doi: 10.1371/journal.pgen.1000264

226. Alexandrov LB, Nik-Zainal S, Wedge DC, Aparicio SA, Behjati $S$, Biankin AV, Bignell GR, Bolli N, Borg A, Borresen-Dale AL, Boyault $S$, Burkhardt B, Butler AP, Caldas C, Davies HR, Desmedt C, Eils R, Eyfjord JE, Foekens JA, Greaves M, Hosoda F, Hutter B, llicic T, Imbeaud S, Imielinski M, Jager N, Jones DT, Jones D, Knappskog S, Kool M, et al. (2013). Signatures of mutational processes in human cancer. Nature 500(7463): 415-421. doi: 10.1038/nature12477

227. Chan K, Roberts SA, Klimczak LJ, Sterling JF, Saini N, Malc EP, Kim J, Kwiatkowski DJ, Fargo DC, Mieczkowski PA, Getz G, Gordenin DA (2015). An APOBEC3A hypermutation signature is distinguishable from the signature of background mutagenesis by $\mathrm{APOBEC} 3 \mathrm{~B}$ in human cancers. Nat Genet 47(9): 1067-1072. doi: 10.1038/ng.3378

228. Nik-Zainal $S$, Alexandrov LB, Wedge DC, Van Loo P, Greenman CD, Raine K, Jones D, Hinton J, Marshall J, Stebbings LA, Menzies A, Martin S, Leung K, Chen L, Leroy C, Ramakrishna M, Rance R, Lau KW, Mudie L, Varela I, McBride DJ, Bignell GR, Cooke SL, Shlien A, Gamble J, Whitmore I, Maddison M, Tarpey PS, Davies HR, Papaemmanuil E, et al. (2012). Mutational processes molding the genomes of 21 breast cancers. Cell 149(5): 979-993. doi: 10.1016/j.cell.2012.04.024

229. Roberts SA, Lawrence MS, Klimczak L, Grimm SA, Fargo D, Stojanov P, Kiezun A, Kryukov GV, Carter SL, Saksena G, Harris S, Shah RR, Resnick MA, Getz G, Gordenin DA (2013). An APOBEC cytidine deaminase mutagenesis pattern is widespread in human cancers. Nat Genet 45(9): 970-976. doi: 10.1038/ng.2702

230. Supek F, Lehner B (2017). Clustered Mutation Signatures Reveal that Error-Prone DNA Repair Targets Mutations to Active Genes. Cell 170(3): 534-547 e523. doi: 10.1016/j.cell.2017.07.003

231. Swanton C, McGranahan N, Starrett GJ, Harris RS (2015). APOBEC Enzymes: Mutagenic Fuel for Cancer Evolution and Heterogeneity. Cancer Discov 5(7): 704-712. doi: 10.1158/2159-8290.CD-15-0344

232. Chan K, Gordenin DA (2015). Clusters of Multiple Mutations: Incidence and Molecular Mechanisms. Annu Rev Genet 49: 243-267. doi: 10.1146/annurev-genet-112414-054714
233. Hoopes JI, Cortez LM, Mertz TM, Malc EP, Mieczkowski PA, Roberts SA (2016). APOBEC3A and APOBEC3B Preferentially Deaminate the Lagging Strand Template during DNA Replication. Cell Rep 14(6): 1273-1282. doi: 10.1016/j.celrep.2016.01.021

234. Lada AG, Kliver SF, Dhar A, Polev DE, Masharsky AE, Rogozin IB, Pavlov YI (2015). Disruption of Transcriptional Coactivator Sub1 Leads to Genome-Wide Re-distribution of Clustered Mutations Induced by APOBEC in Active Yeast Genes. PLoS Genet 11(5): e1005217. doi: 10.1371/journal.pgen.1005217

235. Lada AG, Stepchenkova El, Waisertreiger IS, Noskov VN, Dhar A, Eudy JD, Boissy RJ, Hirano M, Rogozin IB, Pavlov YI (2013). Genomewide mutation avalanches induced in diploid yeast cells by a base analog or an APOBEC deaminase. PLoS Genet 9(9): e1003736. doi: 10.1371/journal.pgen.1003736

236. Taylor BJ, Nik-Zainal S, Wu YL, Stebbings LA, Raine K, Campbell PJ, Rada C, Stratton MR, Neuberger MS (2013). DNA deaminases induce break-associated mutation showers with implication of APOBEC3B and $3 \mathrm{~A}$ in breast cancer kataegis. Elife 2: e00534. doi: 10.7554/elife.00534

237. Taylor BJ, Wu YL, Rada C (2014). Active RNAP pre-initiation sites are highly mutated by cytidine deaminases in yeast, with AID targeting small RNA genes. Elife 3: e03553. doi: 10.7554/eLife.03553

238. Chan K, Resnick MA, Gordenin DA (2013). The choice of nucleotide inserted opposite abasic sites formed within chromosomal DNA reveals the polymerase activities participating in translesion DNA synthesis. DNA Repair 12(11): 878-889. doi 10.1016/j.dnarep.2013.07.008

239. Gibbs PE, McDonald J, Woodgate R, Lawrence CW (2005). The relative roles in vivo of Saccharomyces cerevisiae Pol eta, Pol zeta, Rev1 protein and Pol32 in the bypass and mutation induction of an abasic site, T-T (6-4) photoadduct and T-T cis-syn cyclobutane dimer. Genetics 169(2): 575-582. doi: 10.1534/genetics.104.034611

240. Symington LS (2016). Mechanism and regulation of DNA end resection in eukaryotes. Crit Rev Biochem Mol Biol 51(3):195-212. doi: 10.3109/10409238.2016.1172552

241. Westmoreland JW, Resnick MA (2016). Recombinational repair of radiation-induced double-strand breaks occurs in the absence of extensive resection. Nucleic Acids Res 44(2): 695-704. doi: 10.1093/nar/gkv1109

242. Storici F, Snipe JR, Chan GK, Gordenin DA, Resnick MA (2006). Conservative repair of a chromosomal double-strand break by singlestrand DNA through two steps of annealing. Mol Cell Biol 26(20): 7645-7657. doi: 10.1128/MCB.00672-06

243. Vasan S, Deem A, Ramakrishnan S, Argueso JL, Malkova A (2014). Cascades of genetic instability resulting from compromised breakinduced replication. PLoS Genet 10(2): e1004119. doi: 10.1371/journal.pgen.1004119

244. Westmoreland JW, Resnick MA (2013). Coincident resection at both ends of random, gamma-induced double-strand breaks requires MRX (MRN), Sae2 (Ctp1), and Mre11-nuclease. PLoS Genet 9(3): e1003420. doi: 10.1371/journal.pgen.1003420

245. Rogozin IB, Pavlov YI, Goncearenco A, De S, Lada AG, Poliakov E, Panchenko AR, Cooper DN (2017). Mutational signatures and mutable motifs in cancer genomes. Brief Bioinform. doi: 10.1093/bib/bbx049

246. Saini N, Roberts SA, Sterling JF, Malc EP, Mieczkowski PA, Gordenin DA (2017). APOBEC3B cytidine deaminase targets the nontranscribed strand of tRNA genes in yeast. DNA Repair 53: 4-14. doi: 10.1016/j.dnarep.2017.03.003

247. Morrison A, Johnson AL, Johnston LH, Sugino A (1993). Pathway correcting DNA replication errors in Saccharomyces cerevisiae. EMBO J 12(4): 1467-1473. doi: 10.1002/j.1460-2075.1993.tb05790.x 
248. Haradhvala NJ, Polak P, Stojanov P, Covington KR, Shinbrot E, Hess JM, Rheinbay E, Kim J, Maruvka YE, Braunstein LZ, Kamburov A Hanawalt PC, Wheeler DA, Koren A, Lawrence MS, Getz G (2016). Mutational Strand Asymmetries in Cancer Genomes Reveal Mechanisms of DNA Damage and Repair. Cell 164(3): 538-549. doi: 10.1016/j.cell.2015.12.050

249. Kazanov MD, Roberts SA, Polak P, Stamatoyannopoulos J, Klimczak LJ, Gordenin DA, Sunyaev SR (2015). APOBEC-Induced Cancer Mutations Are Uniquely Enriched in Early-Replicating, Gene-Dense, and Active Chromatin Regions. Cell Rep 13(6): 1103-1109. doi: 10.1016/j.celrep.2015.09.077

250. Shee C, Cox B, Gu F, Luengas EM, Joshi MC, Chiu L, Magnan D, Halliday JA, Frisch RL, Gibson JL, Nehring RB, Do HG, Hernandez M, Li L, Herman C, Hastings PJ, Bates D, Harris RS, Miller KM, Rosenberg SM (2013). Engineered proteins detect spontaneous DNA breaks in human and bacterial cells. eLife 2:e01222. doi: 10.7554/eLife.01222

251. Pennington JM, Rosenberg SM (2007). Spontaneous DNA breakage in single living Escherichia coli cells. Nat Genet 39(6): 797 802. doi: 10.1038/ng2051

252. Nehring RB, Gu F, Lin HY, Gibson JL, Blythe MJ, Wilson R, Bravo Nunez MA, Hastings PJ, Louis EJ, Frisch RL, Hu JC, Rosenberg SM (2016). An ultra-dense library resource for rapid deconvolution of mutations that cause phenotypes in Escherichia coli. Nucleic Acids Res 44(5): e41. doi: 10.1093/nar/gkv1131

253. Cui L, Bikard D (2016). Consequences of Cas9 cleavage in the chromosome of Escherichia coli. Nucleic Acids Res 44(9): 4243-4251. doi: 10.1093/nar/gkw223

254. Wimberly $H$, Shee $C$, Thornton PC, Sivaramakrishnan $P$, Rosenberg SM, Hastings PJ (2013). R-loops and nicks initiate DNA breakage and genome instability in non-growing Escherichia coli. Nat Commun 4: 2115. doi: 10.1038/ncomms3762

255. Sivaramakrishnan P, Sepulveda LA, Halliday JA, Liu J, Nunez MAB, Golding I, Rosenberg SM, Herman C (2017). The transcription fidelity factor GreA impedes DNA break repair. Nature 550(7675): 214-218. doi: 10.1038/nature23907

256. Belenky P, Ye JD, Porter CB, Cohen NR, Lobritz MA, Ferrante $T$, Jain S, Korry BJ, Schwarz EG, Walker GC, Collins JJ (2015). Bactericidal Antibiotics Induce Toxic Metabolic Perturbations that Lead to Cellular Damage. Cell Rep 13(5): 968-980. doi: 10.1016/j.celrep.2015.09.059

257. Jang S, Harshey RM (2015). Repair of transposable phage Mu DNA insertions begins only when the $E$. coli replisome collides with the transpososome. Mol Microbiol 97(4): 746-758. doi: 10.1111/mmi.13061

258. Flatten I, Helgesen E, Pedersen IB, Waldminghaus T, Rothe C, Taipale R, Johnsen L, Skarstad K (2017). Phenotypes of dnaXE145A Mutant Cells Indicate that the Escherichia coli Clamp Loader Has a Role in the Restart of Stalled Replication Forks. J Bacteriol 199(24): e00412-17. doi: 10.1128/JB.00412-17

259. Pedersen IB, Helgesen E, Flatten I, Fossum-Raunehaug S, Skarstad $\mathrm{K}$ (2017). SeqA structures behind Escherichia coli replication forks affect replication elongation and restart mechanisms. Nucleic Acids Res 45(11): 6471-6485. doi: 10.1093/nar/gkx263

260. Xia J, Chen LT, Mei Q, Ma CH, Halliday JA, Lin HY, Magnan D, Pribis JP, Fitzgerald DM, Hamilton HM, Richters $M$, Nehring RB, Shen $X$, Li L, Bates D, Hastings PJ, Herman C, Jayaram M, Rosenberg SM (2016). Holliday junction trap shows how cells use recombination and a junction-guardian role of RecQ helicase. Sci Adv 2(11): e1601605. doi: $10.1126 /$ sciadv.1601605

261. Komor AC, Zhao KT, Packer MS, Gaudelli NM, Waterbury AL, Koblan LW, Kim YB, Badran AH, Liu DR (2017). Improved base excision repair inhibition and bacteriophage Mu Gam protein yields C:G-to-T:A base editors with higher efficiency and product purity. Sci Adv 3(8): eaao4774. doi: 10.1126/sciadv.aao4774

262. Tsouroula K, Furst A, Rogier M, Heyer V, Maglott-Roth A, Ferrand A, Reina-San-Martin B, Soutoglou E (2016). Temporal and Spatial Uncoupling of DNA Double Strand Break Repair Pathways within Mammalian Heterochromatin. Mol Cell 63(2): 293-305. doi: 10.1016/j.molcel.2016.06.002

263. Zhang CZ, Spektor A, Cornils H, Francis JM, Jackson EK, Liu S, Meyerson M, Pellman D (2015). Chromothripsis from DNA damage in micronuclei. Nature 522(7555): 179-184. doi: 10.1038/nature14493

264. Magner DB, Blankschien MD, Lee JA, Pennington JM, Lupski JR, Rosenberg SM (2007). RecQ promotes toxic recombination in cells lacking recombination intermediate-removal proteins. Mol Cell 26(2): 273-286. doi: 10.1016/j.molcel.2007.03.012

265. West SC (1997). Processing of recombination intermediates by the RuvABC proteins. Annu Rev Genet 31: 213-244. doi: 10.1146/annurev.genet.31.1.213

266. Dickman MJ, Ingleston SM, Sedelnikova SE, Rafferty JB, Lloyd RG, Grasby JA, Hornby DP (2002). The RuvABC resolvasome. Eur J Biochem 269(22): 5492-5501. doi: 10.1046/j.1432-1033.2002.03250.x

267. Fogg JM, Schofield MJ, White MF, Lilley DM (1999). Sequence and functional-group specificity for cleavage of DNA junctions by RuvC of Escherichia coli. Biochemistry 38(35): 11349-11358. doi: 10.1021/bi990926n

268. Kuzminov A (1995). Collapse and repair of replication forks in Escherichia coli. Mol Microbiol 16(3): 373-384. doi: 10.1111/j.1365 2958.1995.tb02403.x

269. Motamedi MR, Szigety SK, Rosenberg SM (1999). Double-strandbreak repair recombination in Escherichia coli: physical evidence for a DNA replication mechanism in vivo. Genes Dev 13(21): 2889-2903. doi: 10.1101/gad.13.21.2889

270. Schmutz I, Timashev L, Xie W, Patel DJ, de Lange T (2017). TRF2 binds branched DNA to safeguard telomere integrity. Nat Struct Mol Biol 24(9): 734-742. doi: 10.1038/nsmb.3451

271. Landt SG, Marinov GK, Kundaje A, Kheradpour P, Pauli F, Batzoglou S, Bernstein BE, Bickel P, Brown JB, Cayting P, Chen Y, DeSalvo G, Epstein C, Fisher-Aylor KI, Euskirchen G, Gerstein M, Gertz J, Hartemink AJ, Hoffman MM, lyer VR, Jung YL, Karmakar S, Kellis M, Kharchenko PV, Li Q, Liu T, Liu XS, Ma L, Milosavljevic A, Myers RM, et al. (2012). ChIP-seq guidelines and practices of the ENCODE and modENCODE consortia. Genome Res 22(9): 1813-1831. doi: 10.1101/gr.136184.111

272. Bonocora RP, Fitzgerald DM, Stringer AM, Wade JT (2013). Noncanonical protein-DNA interactions identified by ChIP are not artifacts. BMC Genomics 14: 254. doi: 10.1186/1471-2164-14-254

273. Stracy M, Lesterlin C, Garza de Leon F, Uphoff S, Zawadzki P, Kapanidis AN (2015). Live-cell superresolution microscopy reveals the organization of RNA polymerase in the bacterial nucleoid. Proc Natl Acad Sci U S A 112(32):E4390-9. doi: 10.1073/pnas.1507592112

274. Uphoff S, Reyes-Lamothe R, Garza de Leon F, Sherratt DJ, Kapanidis AN (2013). Single-molecule DNA repair in live bacteria. Proc Natl Acad Sci U S A 110(20): 8063-8068. doi: 10.1073/pnas.1301804110

275. Uphoff S, Sherratt DJ, Kapanidis AN (2014). Visualizing proteinDNA interactions in live bacterial cells using photoactivated singlemolecule tracking. J Vis Exp 85. doi: 10.3791/51177

276. Betzig $E$, Patterson $G H$, Sougrat $R$, Lindwasser OW, Olenych $S$, Bonifacino JS, Davidson MW, Lippincott-Schwartz J, Hess HF (2006). Imaging intracellular fluorescent proteins at nanometer resolution. Science 313(5793): 1642-1645. doi: 10.1126/science.1127344 
277. Manley S, Gillette JM, Patterson GH, Shroff H, Hess HF, Betzig E, Lippincott-Schwartz J (2008). High-density mapping of single-molecule trajectories with photoactivated localization microscopy. Nat Methods 5(2): 155-157. doi: 10.1038/nmeth.1176

278. Stracy M, Jaciuk M, Uphoff S, Kapanidis AN, Nowotny M, Sherratt DJ, Zawadzki $P$ (2016). Single-molecule imaging of UvrA and UvrB recruitment to DNA lesions in living Escherichia coli. Nat Commun 7: 12568. doi: $10.1038 /$ ncomms 12568

279. Persson F, Linden M, Unoson C, Elf J (2013). Extracting intracellular diffusive states and transition rates from single-molecule tracking data. Nat Methods 10(3): 265-269. doi: 10.1038/nmeth.2367

280. Liao Y, Schroeder JW, Gao B, Simmons LA, Biteen JS (2015). Single-molecule motions and interactions in live cells reveal target search dynamics in mismatch repair. Proc Natl Acad Sci U S A 112(50): E6898-6906. doi: 10.1073/pnas.1507386112

281. English BP, Hauryliuk V, Sanamrad A, Tankov S, Dekker NH, Elf J (2011). Single-molecule investigations of the stringent response machinery in living bacterial cells. Proc Natl Acad Sci U S A 108(31): E365-373. doi: 10.1073/pnas.1102255108

282. Bakshi S, Siryaporn A, Goulian M, Weisshaar JC (2012). Superresolution imaging of ribosomes and RNA polymerase in live Escherichia coli cells. Mol Microbiol 85(1): 21-38. doi: 10.1111/j.13652958.2012.08081.x

283. Sanamrad A, Persson F, Lundius EG, Fange D, Gynna AH, Elf J (2014). Single-particle tracking reveals that free ribosomal subunits are not excluded from the Escherichia coli nucleoid. Proc Natl Acad Sci U S A 111(31): 11413-11418. doi: 10.1073/pnas.1411558111

284. Rhodes J, Mazza D, Nasmyth K, Uphoff S (2017). Scc2/Nipbl hops between chromosomal cohesin rings after loading. Elife 6: e30000. doi: $10.7554 /$ eLife. 30000

285. Sarkar P, Switzer A, Peters C, Pogliano J, Wigneshweraraj S (2017). Phenotypic consequences of RNA polymerase dysregulation in Escherichia coli. Nucleic Acids Res 45(19): 11131-11143. doi: 10.1093/nar/gkx733

286. Lisby $M$, Rothstein $R$ (2015). Cell biology of mitotic recombination. Cold Spring Harb Perspect Biol 7(3): a016535. doi: 10.1101/cshperspect.a016535

287. Crickard JB, Kaniecki K, Kwon Y, Sung P, Lisby M, Greene EC (2018). Regulation of Hed1 and Rad54 binding during maturation of the meiosis-specific presynaptic complex. EMBO J 37(7): e98728. doi: 10.15252/embj.201798728

288. Dross N, Spriet C, Zwerger M, Muller G, Waldeck W, Langowski J (2009). Mapping eGFP oligomer mobility in living cell nuclei. PLoS One 4(4): e5041. doi: 10.1371/journal.pone.0005041

289. Jorgensen $P$, Edgington NP, Schneider BL, Rupes I, Tyers $M$, Futcher $B$ (2007). The size of the nucleus increases as yeast cells grow. Mol Biol Cell 18(9): 3523-3532. doi: 10.1091/mbc.E06-10-0973

290. Silva S, Gallina I, Eckert-Boulet N, Lisby M (2012). Live Cell Microscopy of DNA Damage Response in Saccharomyces cerevisiae. Methods Mol Biol 920: 433-443. doi: 10.1007/978-1-61779-998-3_30

291. Lisby M, Rothstein R, Mortensen UH (2001). Rad52 forms DNA repair and recombination centers during $S$ phase. Proc Natl Acad Sci USA 98(15): 8276-8282. doi: 10.1073/pnas.121006298

292. Lisby M, Mortensen UH, Rothstein R (2003). Colocalization of multiple DNA double-strand breaks at a single Rad52 repair centre. Nat Cell Biol 5(6): 572-577. doi: 10.1038/ncb997

293. Heim R, Cubitt AB, Tsien RY (1995). Improved green fluorescence. Nature 373(6516): 663-664. doi: 10.1038/373663b0
294. Ormo M, Cubitt AB, Kallio K, Gross LA, Tsien RY, Remington SJ (1996). Crystal structure of the Aequorea victoria green fluorescent protein. Science 273(5280): 1392-1395. doi: 10.1126/science. 273.5280 .1392

295. De Wulf P, McAinsh AD, Sorger PK (2003). Hierarchical assembly of the budding yeast kinetochore from multiple subcomplexes. Genes Dev 17(23): 2902-2921. doi: 10.1101/gad.1144403

296. Sinnecker D, Voigt P, Hellwig N, Schaefer M (2005). Reversible photobleaching of enhanced green fluorescent proteins. Biochemistry 44(18): 7085-7094. doi: 10.1021/bi047881x

297. Meister P, Gehlen LR, Varela E, Kalck V, Gasser SM (2010) Visualizing yeast chromosomes and nuclear architecture. Methods Enzymol 470: 535-567. doi: 10.1016/S0076-6879(10)70021-5

298. Horigome C, Dion V, Seeber A, Gehlen LR, Gasser SM (2015). Visualizing the spatiotemporal dynamics of DNA damage in budding yeast. Methods Mol Biol 1292: 77-96. doi: 10.1007/978-1-4939-25223_6

299. Sage D, Neumann FR, Hediger F, Gasser SM, Unser M (2005). Automatic tracking of individual fluorescence particles: application to the study of chromosome dynamics. IEEE Trans Image Process 14(9): 1372-1383. doi: 10.1109/tip.2005.852787

300. Amitai A, Seeber A, Gasser SM, Holcman D (2017). Visualization of Chromatin Decompaction and Break Site Extrusion as Predicted by Statistical Polymer Modeling of Single-Locus Trajectories. Cell Rep 18(5): 1200-1214. doi: 10.1016/j.celrep.2017.01.018

301. Mine-Hattab J, Recamier V, Izeddin I, Rothstein R, Darzacq X (2017). Multi-scale tracking reveals scale-dependent chromatin dynamics after DNA damage. Mol Biol Cell 28(23): 3323-3332. doi: 10.1091/mbc.E17-05-0317

302. Dion V, Kalck V, Seeber A, Schleker T, Gasser SM (2013). Cohesin and the nucleolus constrain the mobility of spontaneous repair foci. EMBO Rep 14(11): 984-991. doi: 10.1038/embor.2013.142

303. Neumann FR, Hediger, F., Taddei, A., Gasser, S. M. (2006). Tracking individual Chromosomes with integrated arrays of lacop sites and GFP-loci reporessor: Analyzing position and dynamics of chromosomal loci in Saccharomyces cerevisiae. In: Cells JE, editor, Cell Biology: A Laboratory Handbook (Third Edition). Elsevier Science; pp 359-367.

304. Guerin T, Benichou O, Voituriez R (2012). Non-Markovian polymer reaction kinetics. Nat Chem 4(7): 568-573. doi 10.1038/nchem.1378

305. Robinett CC, Straight A, Li G, Willhelm C, Sudlow G, Murray A Belmont AS (1996). In vivo localization of DNA sequences and visualization of large-scale chromatin organization using lac operator/repressor recognition. J Cell Biol 135(6 Pt 2): 1685-1700. doi: 10.1083/jcb.135.6.1685

306. Possoz C, Filipe SR, Grainge I, Sherratt DJ (2006). Tracking of controlled Escherichia coli replication fork stalling and restart at repressor-bound DNA in vivo. EMBO J 25(11): 2596-2604. doi: 10.1038/sj.emboj.7601155

307. Sofueva S, Osman F, Lorenz A, Steinacher R, Castagnetti S, Ledesma J, Whitby MC (2011). Ultrafine anaphase bridges, broken DNA and illegitimate recombination induced by a replication fork barrier. Nucleic Acids Res 39(15): 6568-6584. doi: 10.1093/nar/gkr340

308. Jacome A, Fernandez-Capetillo O (2011). Lac operator repeats generate a traceable fragile site in mammalian cells. EMBO Rep 12(10): 1032-1038. doi: 10.1038/embor.2011.158

309. Dubarry M, Loiodice I, Chen CL, Thermes C, Taddei A (2011). Tight protein-DNA interactions favor gene silencing. Genes Dev 25(13): 1365-1370. doi: 10.1101/gad.611011 
310. Saad H, Gallardo F, Dalvai M, Tanguy-le-Gac N, Lane D, Bystricky K (2014). DNA Dynamics during Early Double-Strand Break Processing Revealed by Non-Intrusive Imaging of Living Cells. PLoS Genet 10(3): e1004187. doi: 10.1371/journal.pgen.1004187

311. Heun P, Laroche T, Shimada K, Furrer P, Gasser SM (2001). Chromosome dynamics in the yeast interphase nucleus. Science 294(5549): 2181-2186. doi: 10.1126/science.1065366

312. Mine-Hattab J, Rothstein R (2012). Increased chromosome mobility facilitates homology search during recombination. Nat Cell Biol 14(5): 510-517. doi: 10.1038/ncb2472

313. Marshall WF, Straight A, Marko JF, Swedlow J, Dernburg A Belmont A, Murray AW, Agard DA, Sedat JW (1997). Interphase chromosomes undergo constrained diffusional motion in living cells. Curr Biol 7(12): 930-939. doi: 10.1016/s0960-9822(06)00412-x

314. Saxton MJ, Jacobson K (1997). Single-particle tracking: applications to membrane dynamics. Annu Rev Biophys Biomol Struct 26: 373-399. doi: 10.1146/annurev.biophys.26.1.373

315. Barkai E, Garini Y, Metzler R (2012). Strange kinetics of single molecules in living cells. Physics today 65(8): 29-35. doi: 10.1063/pt.3.1677

316. Metzler R, Jeon JH, Cherstvy AG, Barkai E (2014). Anomalous diffusion models and their properties: non-stationarity, nonergodicity, and ageing at the centenary of single particle tracking. Phys Chem Chem Phys 16(44): 24128-24164. doi: 10.1039/c4cp03465a

317. Ben-Avraham DH, S. (2000). Diffusion and reactions in fractals and disordered systems. Cambridge University Press. doi: 10.1017/CBO9780511605826

318. Michalet X (2010). Mean square displacement analysis of singleparticle trajectories with localization error: Brownian motion in an isotropic medium. Phys Rev E Stat Nonlin Soft Matter Phys 82(4 Pt 1): 041914. doi: 10.1103/physreve.82.041914

319. Kepten E, Bronshtein I, Garini Y (2013). Improved estimation of anomalous diffusion exponents in single-particle tracking experiments. Phys Rev E Stat Nonlin Soft Matter Phys 87(5): 052713. doi: 10.1103/physreve.87.052713

320. Bronstein I, Israel Y, Kepten E, Mai S, Shav-Tal Y, Barkai E, Garini Y (2009). Transient anomalous diffusion of telomeres in the nucleus of mammalian cells. Phys Rev Lett 103(1): 018102. doi: 10.1103/physrevlett.103.018102

321. Miné-Hattab J, Rothstein R (2013). DNA in motion during doublestrand break repair. Trends Cell Biol 23(11): 529-536. doi: 10.1016/j.tcb.2013.05.006

322. Dion V, Kalck V, Horigome C, Towbin BD, Gasser SM (2012). Increased mobility of double-strand breaks requires Mec1, Rad9 and the homologous recombination machinery. Nat Cell Biol 14(5): 502509. doi: $10.1038 /$ ncb2465

323. Dimitrova N, Chen YC, Spector DL, de Lange T (2008). 53BP1 promotes non-homologous end joining of telomeres by increasing chromatin mobility. Nature 456(7221): 524-528. doi: $10.1038 /$ nature07433

324. Chiolo I, Minoda A, Colmenares SU, Polyzos A, Costes SV, Karpen GH (2011). Double-strand breaks in heterochromatin move outside of a dynamic HP1a domain to complete recombinational repair. Cell 144(5): 732-744. doi: 10.1016/j.cell.2011.02.012

325. Zeman MK, Cimprich KA (2014). Causes and consequences of replication stress. Nat Cell Biol 16(1): 2-9. doi: 10.1038/ncb2897

326. Larsen NB, Hickson ID, Mankouri HW (2018). A Molecular Toolbox to Engineer Site-Specific DNA Replication Perturbation.
Methods Mol Biol 1672: 295-309. doi: 10.1007/978-1-4939-73064_20

327. Lambert S, Watson A, Sheedy DM, Martin B, Carr AM (2005). Gross chromosomal rearrangements and elevated recombination at an inducible site-specific replication fork barrier. Cell 121(5): 689-702. doi:10.1016/j.cell.2005.03.022

328. Nguyen MO, Jalan M, Morrow CA, Osman F, Whitby MC (2015). Recombination occurs within minutes of replication blockage by RTS1 producing restarted forks that are prone to collapse. Elife 4: e04539. doi: 10.7554/eLife.04539

329. Willis NA, Chandramouly G, Huang B, Kwok A, Follonier C, Deng C, Scully $R$ (2014). BRCA1 controls homologous recombination at Tus/Ter-stalled mammalian replication forks. Nature 510(7506): 556559. doi: 10.1038/nature13295

330. Su XA, Dion V, Gasser SM, Freudenreich CH (2015). Regulation of recombination at yeast nuclear pores controls repair and triplet repeat stability. Genes Dev 29(10): 1006-1017. doi: 10.1101/gad.256404.114

331. Dalgaard JZ, Klar AJ (2001). A DNA replication-arrest site RTS1 regulates imprinting by determining the direction of replication at mat1 in S. pombe. Genes Dev 15(16): 2060-2068. doi: 10.1101/gad.200801

332. Miyabe I, Mizuno K, Keszthelyi A, Daigaku Y, Skouteri M, Mohebi S, Kunkel TA, Murray JM, Carr AM (2015). Polymerase delta replicates both strands after homologous recombination-dependent fork restart. Nat Struct Mol Biol 22(11): 932-938. doi: 10.1038/nsmb.3100

333. Ait Saada A, Teixeira-Silva A, Iraqui I, Costes A, Hardy J, Paoletti G, Freon K, Lambert SAE (2017). Unprotected Replication Forks Are Converted into Mitotic Sister Chromatid Bridges. Mol Cell 66(3): 398 410 e394. doi: 10.1016/j.molcel.2017.04.002

334. Velve-Casquillas G, Costa J, Carlier-Grynkorn F, Mayeux A, Tran PT (2010). A fast microfluidic temperature control device for studying microtubule dynamics in fission yeast. Methods Cell Biol 97: 185-201. doi: 10.1016/S0091-679X(10)97011-8

335. Teixeira-Silva A, Ait Saada A, Hardy J, Iraqui I, Nocente MC, Freon $\mathrm{K}$, Lambert SAE (2017). The end-joining factor Ku acts in the endresection of double strand break-free arrested replication forks. Nat Commun 8(1): 1982. doi: 10.1038/s41467-017-02144-5

336. Mizuno K, Miyabe I, Schalbetter SA, Carr AM, Murray JM (2013) Recombination-restarted replication makes inverted chromosome fusions at inverted repeats. Nature 493(7431): 246-249. doi: 10.1038/nature11676

337. Hustedt N, Durocher D (2016). The control of DNA repair by the cell cycle. Nat Cell Biol 19(1): 1-9. doi: 10.1038/ncb3452

338. Symington LS (2014). End resection at double-strand breaks: mechanism and regulation. Cold Spring Harb Perspect Biol 6(8). doi: 10.1101/cshperspect.a016436

339. Daley JM, Sung P (2014). 53BP1, BRCA1, and the choice between recombination and end joining at DNA double-strand breaks. Mol Cell Biol 34(8): 1380-1388. doi: 10.1128/MCB.01639-13

340. Andres SN, Williams RS (2017). CtIP/Ctp1/Sae2, molecular form fit for function. DNA Repair 56: 109-117. doi: 10.1016/j.dnarep.2017.06.013

341. Cannavo E, Cejka P (2014). Sae2 promotes dsDNA endonuclease activity within Mre11-Rad50-Xrs2 to resect DNA breaks. Nature 514(7520): 122-125. doi: 10.1038/nature13771

342. Cejka P, Cannavo E, Polaczek P, Masuda-Sasa T, Pokharel S, Campbell JL, Kowalczykowski SC (2010). DNA end resection by Dna2Sgs1-RPA and its stimulation by Top3-Rmi1 and Mre11-Rad50-Xrs2. Nature 467(7311): 112-116. doi: 10.1038/nature09355 
343. Kowalczykowski SC (2015). An Overview of the Molecular Mechanisms of Recombinational DNA Repair. Cold Spring Harb Perspect Biol 7(11). doi: 10.1101/cshperspect.a016410

344. Limbo O, Chahwan C, Yamada Y, de Bruin RA, Wittenberg C, Russell $P$ (2007). Ctp1 is a cell-cycle-regulated protein that functions with Mre11 complex to control double-strand break repair by homologous recombination. Mol Cell 28(1): 134-146. doi: 10.1016/j.molcel.2007.09.009

345. Chen X, Cui D, Papusha A, Zhang X, Chu CD, Tang J, Chen K, Pan X, Ira $G$ (2012). The Fun30 nucleosome remodeller promotes resection of DNA double-strand break ends. Nature 489(7417): 576-580. doi: 10.1038/nature11355

346. Croteau DL, Popuri V, Opresko PL, Bohr VA (2014). Human RecQ helicases in DNA repair, recombination, and replication. Annu Rev Biochem 83: 519-552. doi: 10.1146/annurev-biochem-060713-035428

347. Nimonkar AV, Genschel J, Kinoshita E, Polaczek P, Campbell JL, Wyman C, Modrich P, Kowalczykowski SC (2011). BLM-DNA2-RPAMRN and EXO1-BLM-RPA-MRN constitute two DNA end resection machineries for human DNA break repair. Genes Dev 25(4): 350-362. doi: 10.1101/gad.2003811

348. Zhu Z, Chung WH, Shim EY, Lee SE, Ira G (2008). Sgs1 helicase and two nucleases Dna2 and Exo1 resect DNA double-strand break ends. Cell 134(6): 981-994. doi: 10.1016/j.cell.2008.08.037

349. Langerak P, Mejia-Ramirez E, Limbo O, Russell P (2011). Release of Ku and MRN from DNA ends by Mre11 nuclease activity and Ctp1 is required for homologous recombination repair of double-strand breaks. PLoS Genet 7(9): e1002271. doi: 10.1371/journal.pgen.1002271

350. Leland BA, Chen AC, Zhao AY, Wharton RC, King MC (2018). Rev7 and 53BP1/Crb2 prevent RecQ helicase-dependent hyper-resection of DNA double-strand breaks. Elife 7: e33402. doi: 10.7554/eLife.33402

351. Lisby M, Barlow JH, Burgess RC, Rothstein R (2004). Choreography of the DNA damage response: spatiotemporal relationships among checkpoint and repair proteins. Cell 118(6): 699713. doi: 10.1016/j.cell.2004.08.015

352. Ferrari M, Dibitetto D, De Gregorio G, Eapen VV, Rawal CC, Lazzaro F, Tsabar M, Marini F, Haber JE, Pellicioli A (2015). Functional interplay between the 53BP1-ortholog Rad9 and the Mre11 complex regulates resection, end-tethering and repair of a double-strand break. PLoS Genet 11(1): e1004928. doi: 10.1371/journal.pgen.1004928

353. Chapman JR, Barral P, Vannier JB, Borel V, Steger M, Tomas-Loba A, Sartori AA, Adams IR, Batista FD, Boulton SJ (2013). RIF1 is essential for 53BP1-dependent nonhomologous end joining and suppression of DNA double-strand break resection. Mol Cell 49(5): 858-871. doi: 10.1016/j.molcel.2013.01.002

354. Ochs F, Somyajit K, Altmeyer M, Rask MB, Lukas J, Lukas C (2016). 53BP1 fosters fidelity of homology-directed DNA repair. Nat Struct Mol Biol 23(8):714-21. doi: 10.1038/nsmb.3251

355. Zimmermann M, Lottersberger F, Buonomo SB, Sfeir A, de Lange $T$ (2013). 53BP1 regulates DSB repair using Rif1 to control 5 ' end resection. Science 339(6120): 700-704. doi: 10.1126/science.1231573

356. Horigome C, Bustard DE, Marcomini I, Delgoshaie N, TsaiPflugfelder M, Cobb JA, Gasser SM (2016). PolySUMOylation by Siz2 and Mms21 triggers relocation of DNA breaks to nuclear pores through the SIx5/SIx8 STUbL. Genes Dev 30(8): 931-945. doi: $10.1101 /$ gad.277665.116

357. Horigome C, Oma Y, Konishi T, Schmid R, Marcomini I, Hauer MH, Dion V, Harata M, Gasser SM (2014). SWR1 and INO80 chromatin remodelers contribute to DNA double-strand break perinuclear anchorage site choice. Mol Cell 55(4): 626-639. doi: 10.1016/j.molcel.2014.06.027

358. Kalocsay M, Hiller NJ, Jentsch S (2009). Chromosome-wide Rad51 spreading and SUMO-H2A.Z-dependent chromosome fixation in response to a persistent DNA double-strand break. Mol Cell 33(3): 335-343. doi: 10.1016/j.molcel.2009.01.016

359. Nagai S, Dubrana K, Tsai-Pflugfelder M, Davidson MB, Roberts TM, Brown GW, Varela E, Hediger F, Gasser SM, Krogan NJ (2008). Functional targeting of DNA damage to a nuclear pore-associated SUMO-dependent ubiquitin ligase. Science 322(5901): 597-602. doi: 10.1126/science. 1162790

360. Oza P, Jaspersen SL, Miele A, Dekker J, Peterson CL (2009). Mechanisms that regulate localization of a DNA double-strand break to the nuclear periphery. Genes Dev 23(8): 912-927. doi: 10.1101/gad.1782209

361. Ryu T, Spatola B, Delabaere L, Bowlin K, Hopp H, Kunitake R, Karpen GH, Chiolo I (2015). Heterochromatic breaks move to the nuclear periphery to continue recombinational repair. Nat Cell Biol 17(11): 1401-1411. doi: 10.1038/ncb3258

362. Swartz RK, Rodriguez EC, King MC (2014). A role for nuclear envelope-bridging complexes in homology-directed repair. Mol Biol Cell 25(16): 2461-2471. doi: 10.1091/mbc.E13-10-0569

363. Watson AT, Daigaku Y, Mohebi S, Etheridge TJ, Chahwan C, Murray JM, Carr AM (2013). Optimisation of the Schizosaccharomyces pombe urg1 expression system. PLoS One 8(12): e83800. doi: 10.1371/journal.pone.0083800

364. Lang GI, Murray AW (2011). Mutation rates across budding yeast chromosome VI are correlated with replication timing. Genome Biol Evolut 3: 799-811. doi: 10.1093/gbe/evr054

365. House NC, Yang JH, Walsh SC, Moy JM, Freudenreich CH (2014). NuA4 Initiates Dynamic Histone H4 Acetylation to Promote HighFidelity Sister Chromatid Recombination at Postreplication Gaps. Mol Cell 55(6): 818-828. doi: 10.1016/j.molcel.2014.07.007

366. Nguyen JH, Viterbo D, Anand RP, Verra L, Sloan L, Richard GF, Freudenreich $\mathrm{CH}$ (2017). Differential requirement of Srs2 helicase and Rad51 displacement activities in replication of hairpin-forming CAG/CTG repeats. Nucleic Acids Res 45(8):4519-4531. doi: 10.1093/nar/gkx088.

367. Yang JH, Freudenreich $\mathrm{CH}$ (2010). The Rtt109 histone acetyltransferase facilitates error-free replication to prevent CAG/CTG repeat contractions. DNA Repair 9(4): 414-420. doi: 10.1016/j.dnarep.2009.12.022

368. Huh WK, Falvo JV, Gerke LC, Carroll AS, Howson RW, Weissman JS, O'Shea EK (2003). Global analysis of protein localization in budding yeast. Nature 425(6959): 686-691. doi: 10.1038/nature02026

369. Miné-Hattab J, Rothstein R (2012). [DNA repair: finding the perfect match]. Med Sci 28(8-9): 714-716. doi: 10.1051/medsci/2012288014 